\title{
Modelos Aditivos Binomiais Negativos
}

\section{Jacqueline Sant' Eufemia David}

\author{
Dissertação apresentada no \\ Instituto de Matemática e Estatística \\ da Universidade de São Paulo \\ para obtenção do título de \\ Mestre em Estatística
}

\section{Área de Concentração: Estatistica Orientadora: Silvia Lopes de Paula Ferrari}

Apoio Financeiro: FAPESP

- São Paulo, Ábril de 2003 - 


\section{Agradecimentos}

À minha orientadora Silvia Lopes de Paula Ferrari, pela inestimável confiança que em mim depositou e pela infinita disponibilidade em partilhar comigo o seu saber e rigor científico, os quais foram imprescindíveis para minha formação como estatística. Meus mais sinceros agradecimentos por sua orientação precisa, pelas valiosíssimas críticas e sugestões que me foi fazendo ao longo do trabalho de investigação e redação dessa dissertação, e pelos inúmeros momentos de conforto, apoio e estímulo, sempre indicando a direção a ser tomada nos momentos de maior dificuldade. Estou-lhe eternamente grata por isso.

Aos professores do Departamento de Estatística do IME-USP que contribuíram para minha formação, e especialmente à professora Carmen Diva Saldiva de André, pelo exemplo, confiança, incentivo e amizade ao longo dessa etapa.

Aos meus pais, Solange e Abel, pelo exemplo de vida, pelo estímulo, apoio e paciência incondicionais, e pela excitação e orgulho com que sempre reagiram aos resultados dessa jornada. Espero ter a capacidade de lhes retribuir um pouco do que fizeram por mim.

Ao meu querido Dennis, pela imensa compreensão e ternura, por toda a força, confiança e inspiração sempre manifestadas apesar do "débito" de atenção em muitas fases dessa jornada.

Aos meus irmãos Michele e Daniel, e ao grande amigo e cunhado Ferdinando, pelo apoio constante e pela grande amizade e torcida que sempre me dedicaram.

Às queridas amigas Bianca e Kelly, pelas inúmeras e preciosas discussões, pelos trabalhos compartilhados, pelo constante incentivo e pela enorme amizade. Aos amigos Elaine, Fábio e Marcos, por estarem sempre presentes.

Às amigas Liliam e Gleice do Laboratório de Poluição Atmosférica Experimental da Faculdade de Medicina da USP, pelo fornecimento dos dados, pelas discussões e disponibilidade que sempre demonstraram.

Aos amigos que fiz durante esta jornada, em especial à Audrey e ao Francisco, pela agradável convivência e pelo companheirismo e incentivo.

À FAPESP, pelos recursos disponibilizados para elaboração dessa dissertação.

Por fim, a Deus e ao Menino Jesus de Praga, pela luz, força e coragem ao longo de toda essa jornada. 
Aos meus pais Solange e Abel, e ao meu querido Dennis. 
Este exemplar corresponde à redação final da dissertação devidamente corrigida e defendida por Jacqueline Sant' Eufemia David e aprovada pela comissão julgadora.

São Paulo, 25 de abril de 2003.

Banca examinadora:

- Profa. Dra. Silvia Lopes de Paula Ferrari (orientadora) - IME/USP

- Profa. Dra. Carmen Diva Saldiva de André - IME/USP

- Profa. Dra. Antonieta D'Alcântara de Queiroz Peres - UNIRIO 


\section{Resumo}

Os modelos lineares generalizados (MLG) são uma ampla classe de modelos de regressão. No entanto, as suposições impostas por esses modelos não são adequadas, por exemplo, para a análise de dados de contagem superdispersos. Um dos modelos de superdispersão muito utilizado é o modelo de regressão binomial negativo. Se um certo parâmetro desse modelo é conhecido, ele faz parte da classe dos MLG's. No entanto, a suposição de que tal parâmetro é conhecido é geralmente irreal e métodos adequados de inferência nesses modelos estão descritos na literatura. Os MLG's impõem uma restrição adicional: uma função estritamente monótona da resposta média, a função de ligação, deve estar relacionada a um preditor linear, que envolve parâmetros desconhecidos e as covariáveis. Os modelos aditivos generalizados (MAG) estendem a classe dos MLG's permitindo não linearidade na relação entre uma função da resposta média e as covariáveis, que é modelada através de funções alisadoras não especificadas. Embora os MAG's constituam uma classe mais ampla que os MLG's, eles também não são adequados para a análise de contagens superdispersas. Recentemente, os MAG's foram estendidos para englobar respostas binomiais negativas. Nessa dissertação apresentamos esta extensão e sua implemetação computacional. Apresentamos também uma aplicação desse modelo a dados reais, com ênfase no estudo da relação entre poluição atmosférica e saúde humana na cidade de São Paulo. 


\section{Abstract}

The generalized linear models (GLM) are a wide class of regression models. However, the assumptions imposed by these models are not suitable, for example, to analyze overdispersed count data. One of the most used overdispersion models is the negative binomial regression model. If a certain parameter of this model is known, it belongs to the class of the GLM's. Nevertheless, the assumption that such parameter is known is oftentimes unreal and methods for inference in this model are described in the literature. The GLM's impose an additional restriction: a strictly monotonic function of the mean response, the link function, is assumed to be related to a linear predictor, which involves unknown parameters and covariates. The generalized additive models (GAM) framework extends the class of the GLM's by allowing nonlinearity in the relationship between a function of the mean response and the covariates, which is modeled as an unspecified smooth function. Although the GAM's constitute a wider class than that of the GLM's, they are not suitable to analyze overdispersed counts. Recently, the GAM framework was extended to include negative binomial responses. In this thesis, we present this extension and its computer implementation. We also present an application of this model to real data, with emphasis in the study of the association between air pollution and human health in São Paulo city. 


\section{Sumário}

1 Introdução 1

2 Modelos Lineares Generalizados 4

2.1 Definição . . . . . . . . . . . . . . . . . . . . 4

2.2 Estimação dos Parâmetros . . . . . . . . . . . . . . . 6

2.3 Função Desvio . . . . . . . . . . . . . . . . . . . . . . 7

2.4 Análise Inferencial . . . . . . . . . . . . . . . . . 8

2.5 Técnicas de Diagnóstico . . . . . . . . . . . . . . . . . 10

2.6 Modelo Binomial Negativo . . . . . . . . . . . . . . . . . . 14

2.7 Risco Relativo . . . . . . . . . . . . . . . . . . . . . 24

3 Modelos Aditivos Generalizados $\quad 26$

3.1 Definição . . . . . . . . . . . . . . . . 26

3.2 Alisadores . . . . . . . . . . . . . . . . . . 29

3.3 Estimação . . . . . . . . . . . . . . . . . . . . . 38

3.3 .1 Modelo Aditivo . . . . . . . . . . . . . . . . . . . 38

3.3.2 Modelo Aditivo Generalizado . . . . . . . . . . . . . 42

3.4 Análise Inferencial . . . . . . . . . . . . . . . . . 51

3.5 Seleção dos Parâmetros de Alisamento . . . . . . . . . . . . . . 53

3.6 Técnicas de Diagnóstico . . . . . . . . . . . . . . . 56

3.7 Modelo Aditivo Binomial Negativo . . . . . . . . . . . . . . . . . 59

3.8 Risco Relativo . . . . . . . . . . . . . . . . . . . . . . 71

4 Associação entre poluição atmosférica e saúde em São Paulo 74

$\begin{array}{llr}5 & \text { Conclusões } & 95\end{array}$

$\begin{array}{ll}\text { A Função gam.nb } & 97\end{array}$

$\begin{array}{ll}\text { Referências Bibliográficas } & 99\end{array}$ 


\section{Capítulo 1}

\section{Introdução}

Os modelos lineares generalizados (MLG) são uma ampla classe de modelos de regressão que engloba os modelos normais lineares, os modelos de regressão logística para dados binários ou na forma de proporções, os modelos de regressão de Poisson, entre outros (McCullagh e Nelder, 1989).

O modelo de regressão de Poisson é muito utilizado para descrever a relação entre dados de contagem e um conjunto de variáveis explicativas, mas quando aplicamos um modelo a um conjunto de dados, é preciso ter o cuidado de verificar se este modelo é adequado, ou seja, se fornece um bom ajuste.

Os modelos de regressão de Poisson exigem que, dados os valores das covariáveis, a variância e a média da variável resposta sejam iguais. Tal suposição é, muitas vezes, violada na prática levando ao ajuste inadequado do modelo de Poisson pela variabilidade (dispersão) dos dados ser maior que a predita pelo modelo. Em outras palavras, podemos dizer que o fenômeno de superdispersão ocorre quando é esperada uma distribuição de Poisson para a resposta, porém a variância é maior que a resposta média, ou seja, supõe-se que $Y \sim P(\mu)$, e logo $\mathrm{E}(Y)=\operatorname{Var}(Y)$, porém, os dados dão evidências de que

$$
\operatorname{Var}(Y)>\mathrm{E}(Y)
$$

Existem diferentes possíveis causas para este fenômeno e, em qualquer situação (modelagem), várias delas podem estar envolvidas simultaneamente. Algumas possibilidades são: heterogeneidade das unidades amostrais, não observação de variáveis explicativas de grande importância para a resposta, probabilidade de ocorrência de um certo evento variar com o tempo, existência de correlação entre as unidades amostrais, entre outras. Em alguns casos a superdispersão fica aparente pela natureza dos dados, mas na maioria deles é difícil estabelecer sua causa exata.

Uma conseqüência importante de se ignorar a superdispersão é que os erros 
padrão obtidos através do modelo de regressão de Poisson são incorretos e subestimam a variabilidade dos estimadores de seus parâmetros. Assim, na presença de superdispersão o desvio do modelo de Poisson estará incorreto, e logo, teremos informações incorretas sobre a significância da cada parâmetro, podendo optar por um modelo complexo sem necessidade.

Desta forma, fica claro que o modelo de regressão de Poisson precisa ser aprimorado a fim de controlar esta dispersão extra-Poisson (superdispersão), e os modelos de superdispersão para dados de contagem são uma possível alternativa aos modelos de regressão de Poisson (Hinde e Demétrio, 1998 e David, 2000).

Um dos modelos de superdispersão muito utilizado é o modelo de regressão binomial negativo. Se um determinado parâmetro desse modelo é conhecido, ele faz parte da classe dos modelos lineares generalizados e os métodos de estimação, testes de hipóteses e diagnóstico, válidos para os MLG's podem ser utilizados. No entanto, a suposição de que tal parâmetro é conhecido é geralmente irreal e métodos adequados de inferência nesses modelos foram desenvolvidos (ver, por exemplo, Hinde e Demétrio, 1998, Lawless, 1987 e David, 2000).

Os MLG's impõem ainda uma outra restrição: uma função biunívoca da média da variável resposta, chamada de função de ligação, deve estar relacionada a um preditor linear, que envolve parâmetros desconhecidos e as covariáveis. Os modelos aditivos generalizados (MAG) estendem a classe dos MLG's permitindo não linearidade na relação entre uma função da média da variável resposta e as covariáveis (Hastie e Tibshirani, 1990). Se $x_{1}, \ldots, x_{n}$ são um conjunto de $n$ covariadas de dimensão $p$, onde $x_{i 1} \equiv 1$ é a variável de planejamento associada ao intercepto do modelo para todo $i=1, \ldots, n$, e se $\mu_{i}$ indica a média da variável resposta correspondente à $i$-ésima observação, os MLG's impõem que

$$
\eta_{i}=g\left(\mu_{i}\right)=\beta_{1}+\sum_{j=2}^{p} \beta_{j} x_{i j}
$$

enquanto que os MAG's admitem que

$$
\eta_{i}=g\left(\mu_{i}\right)=\beta_{1}+\sum_{j=2}^{p} f_{j}\left(x_{i j}\right),
$$

onde cada $f_{j}$ é uma função contínua não paramétrica estimada através de curvas de alisamento, também chamadas de alisadores (Hastie e Tibshirani, 1990). Um caso particular desse último é o modelo aditivo generalizado parcialmente linear

$$
\eta_{i}=g\left(\mu_{i}\right)=\beta_{1}+\sum_{j=2}^{p_{1}} \beta_{j} x_{i j}+\sum_{j=p_{1}+1}^{p} f_{j}\left(x_{i j}\right),
$$

onde $2 \leq p_{1}<p$. 
O software S-PLUS 4.5 tem rotinas para inferências tanto nos MLG's quanto nos MAG's.

Os MAG's, embora sejam uma classe mais ampla que os MLG's, não são adequados para a análise de dados de contagem superdispersos. Uma alternativa é, portanto, utilizar um modelo aditivo binomial negativo que admite que, dados os valores das covariáveis, a distribuição da variável resposta é binomial negativa com média que se relaciona com as covariáveis através de (1.1). Se não é admitido que um determinado parâmetro da distribuição binomial negativa é conhecido, o modelo aditivo binomial negativo não faz parte dos MAG's. Recentemente, Thurston, Wand e Wiencke (2000) estenderam os MAG's para englobar respostas binomiais negativas. Como enfatizado pelos autores, a extensão é complicada pelo fato da distribuição binomial negativa envolver dois parâmetros e não fazer parte da família exponencial. Eles indicam os passos para a implementação de um algoritmo iterativo para o ajuste do modelo e mostram como inferências devem ser realizadas com base nesse modelo.

Assim, os objetivos dessa dissertação são:

1. apresentar os principais resultados teóricos acerca dos MLG's e do modelo de regressão binomial negativo;

2. apresentar os principais resultados teóricos acerca dos MAG's;

3. apresentar a extensão dos MAG's para os modelos aditivos binomiais negativos;

4. implementar no S-PLUS 4.5 o algoritmo de ajuste dos modelos aditivos binomiais negativos;

5. aplicar os modelos aditivos binomiais negativos a dados reais, com ênfase numa aplicação em estudo da relação entre poluição atmosférica e saúde humana. 


\section{Capítulo 2}

\section{Modelos Lineares Generalizados}

\subsection{Definição}

Seja $Y$ uma variável aleatória (v.a.) com função densidade de probabilidade (f.d.p.) ou função de probabilidade (f.p.)

$$
f(y ; \theta, \phi)=\exp \left\{\phi^{-1}[y \theta-b(\theta)]+c(y, \phi)\right\}, \quad y \in E,
$$

onde $E$ não depende de $\theta, b(\cdot)$ e $c(\cdot)$ são funções especificadas, $b(\cdot)$ é duplamente diferenciável e $\phi>0$. Se $\phi$ é conhecido, este é um modelo da família exponencial unidimensional com parâmetro canônico (natural) $\theta$. Diversas distribuições importantes tais como as distribuições normal, de Poisson, binomial, gama e normal inversa, são da forma (2.1).

Se $Y$ tem f.d.p. ou f.p. como dado em (2.1), então pode-se mostrar (McCullagh e Nelder, 1989, Seção 2.2.2) que

$$
\mu=\mathrm{E}(Y)=b^{\prime}(\theta)
$$

e

$$
\operatorname{Var}(Y)=\phi b^{\prime \prime}(\theta)
$$

Note que $\operatorname{Var}(Y)$ é o produto de duas quantidades: a função $b^{\prime \prime}(\theta)$, que depende somente do parâmetro canônico (e, portanto, da média de $Y$ ) e que é chamada de função de variância e do chamado parâmetro de dispersão $\phi$, que não depende de $\theta$. A função de variância, considerada como uma função de $\mu$, será denotada por $V(\mu)$. Vale destacar que a função de variância identifica distribuições na família exponencial dada por (2.1).

Suponha agora que $Y_{1}, \ldots, Y_{n}$ sejam $n$ v.a.'s independentes sendo que, para $i=1, \ldots, n, Y_{i}$ tem f.d.p. ou f.p. na forma (2.1) $\operatorname{com} \theta=\theta_{i}$. Suponha ainda 
que a média $\mu_{i}$ de $Y_{i}$ está relacionada com um vetor $\beta=\left(\beta_{1}, \ldots, \beta_{p}\right)^{\top}, p<n$, de parâmetros desconhecidos através de uma função $g(\cdot)$ tal que

$$
g\left(\mu_{i}\right)=\eta_{i},
$$

onde $\eta_{i}$, chamado de preditor linear, é dado por

$$
\eta_{i}=x_{i}^{\top} \beta,
$$

com $x_{i}=\left(x_{i 1}, x_{i 2}, \ldots, x_{i p}\right)^{\top}$ representando um vetor de variáveis preditoras (não aleatórias), e lembrando que $x_{i 1} \equiv 1$ para todo $i=1, \ldots, n$. Assumimos que $g(\cdot)$ é uma função monótona e diferenciável. Tal função é chamada de função de ligação.

Os MLG's são então definidos por (2.1), (2.2) e (2.3) com (2.1) representando o componente aleatório do modelo e (2.2)-(2.3), o componente sistemático.

Note que o MLG's estendem a classe dos modelos normais lineares em duas direções:

1. permitem distribuições não normais para os dados;

2. assumem uma ligação, não necessariamente a indentidade, entre a média $\mu_{i}$ e o preditor linear $\eta_{i}$.

Exemplo 2.1: A distribuição de Poisson produz o modelo padrão para análise de dados de contagem. É largamente empregada quando a variável resposta representa uma contagem do número de eventos de um certo tipo que ocorrem em um intervalo de tempo, superfície ou volume. Geralmente são eventos que apresentam pequena probabilidade de ocorrer em uma amostra particular, mas para um grande número de amostras, esta probabilidade é considerável.

Sejam $Y_{1}, \ldots, Y_{n} n$ v.a.'s independentes tais que $Y_{i} \sim \operatorname{Poisson}\left(\mu_{i}\right)$, ou seja, sua função de probabilidade é

$$
f_{Y_{i}}\left(y_{i} ; \mu_{i}\right)=\frac{e^{-\mu_{i}} \mu_{i}^{y_{i}}}{y_{i} !}, \quad y_{i}=0,1,2, \ldots
$$

Essa distribuição faz parte da família exponencial com $\phi=1, b\left(\theta_{i}\right)=e^{\theta_{i}}, \mu_{i}=e^{\theta_{i}}$, $c\left(y_{i}, \phi\right)=\log y_{i}$ ! e $V\left(\mu_{i}\right)=\mu_{i}$. Logo,

$$
\mathrm{E}\left(Y_{i}\right)=\operatorname{Var}\left(Y_{i}\right)=\mu_{i} .
$$

Assumimos que

$$
\mu_{i}=e^{\eta_{i}}=e^{x_{i}^{\top} \beta}
$$

ou seja,

$$
\eta_{i}=x_{i}^{\top} \beta=\log \mu_{i},
$$


com $x_{i}=\left(1, x_{i 2}, \ldots, x_{i p}\right)^{\top}$ representando um vetor de variáveis preditoras (não aleatórias) e $\beta=\left(\beta_{1}, \beta_{2}, \ldots, \beta_{p}\right)^{\top}$ um vetor de parâmetros desconhecidos.

Vale destacar que este modelo é muito utilizado para análise de dados de contagem, e é conhecido como modelo de regressão de Poisson, que portanto, faz parte da classe dos modelos lineares generalizados. Para um estudo mais detalhado do modelo de regressão de Poisson, ver McCullagh e Nelder (1989, Capítulo 6), Paula (2002, Capítulo 4) ou David (1999, Capítulo 3).

Os MLG's têm desempenhado um papel fundamental na análise estatísitca de dados pois englobam modelos de regressão importantes como o descrito acima, dispõem de um processo iterativo simples de estimação de parâmetros e possibilitam a definição de uma função chamada de função desvio (deviance) que generaliza a soma de quadrados dos resíduos dos modelos normais lineares e que permite a construção de testes de hipóteses de forma análoga aos testes baseados em análise de variância utilizados nos modelos normais lineares.

\subsection{Estimação dos Parâmetros}

Um estimador do vetor de parâmetros $\beta$ pode ser obtido pelo método de máxima verossimilhança. Admitindo que $\phi$ é conhecido, o logaritmo da função de verossimilhança de $\beta$ para a amostra $y=\left(y_{1}, \ldots, y_{n}\right)$ é dado por

$$
l(\beta ; y)=\sum_{i=1}^{n} \frac{y_{i} \theta_{i}}{\phi}-\sum_{i=1}^{n} \frac{b\left(\theta_{i}\right)}{\phi}+\sum_{i=1}^{n} c\left(y_{i}, \phi\right),
$$

lembrando que $g\left(\mu_{i}\right)=g\left(b^{\prime}\left(\theta_{i}\right)\right)=\eta_{i}=x_{i}^{\top} \beta$. A derivada de $l(\beta ; y)$ em relação a $\beta_{j}$ é dada por

$$
\frac{\partial l(\beta ; y)}{\partial \beta_{j}}=\sum_{i=1}^{n} x_{i j}\left(\frac{\partial \mu_{i}}{\partial \eta_{i}}\right) \frac{y_{i}-\mu_{i}}{\operatorname{Var}\left(\mathrm{Y}_{\mathrm{i}}\right)}
$$

O estimador de máxima verossimilhança $\widehat{\beta}$ do vetor de parâmetros $\beta$ corresponde à solução do sistema de equações escore gerado quando igualamos $\partial l(\beta ; y) / \partial \beta_{j}$ a zero, para $j=1, \ldots, p$. Essas equações são, em geral, não lineares e precisam ser resolvidas por algum procedimento iterativo.

O método scoring de Fisher é equivalente ao procedimento iterativo de mínimos quadrados reponderados, que leva a

$$
\beta^{(m+1)}=\left(X^{\top} W^{(m)} X\right)^{-1} X^{\top} W^{(m)} z^{(m)},
$$


onde $X$ representa uma matriz $n \times p$ de posto completo cujas linhas são denotadas por $x_{i}^{\top}, i=1, \ldots, n$, e $W^{(m)}$ e $z^{(m)}$ são as estimativas de $W$ e $z$ no passo $m$, sendo

$$
W=\operatorname{diag}\left(w_{1}, \ldots, w_{n}\right)
$$

com

e $z$, o vetor $n \times 1$ de componentes

$$
w_{i}=\left(\frac{d \mu_{i}}{d \eta_{i}}\right)^{2} \frac{1}{V\left(\mu_{i}\right)}
$$

$$
z_{i}=x_{i}^{\top} \beta+\left(y_{i}-\mu_{i}\right) \frac{d \eta_{i}}{d \mu_{i}} .
$$

Nota-se que em cada passo desse processo iterativo $\beta$ é estimado pelo método de mínimos quadrados ponderados em uma regressão de uma variável dependente modificada $z$ em $X$, com pesos $W$ que mudam a cada iteração.

Raramente ocorrem problemas na covergência do processo (2.4). É recomendável usar os próprios dados para obter uma primeira estimativa $\beta^{(0)}$ começando com os "valores ajustados" $\mu_{i}^{(0)}=y_{i}$. Esta é uma boa escolha para o valor inicial, pois reduz o número de passos necessários para que o processo (2.4) convirja. Em alguns casos, correções são necessárias para prevenir, por exemplo, a tentativa de avaliar $\log (0)$ como valor inicial para $\eta$ quando a função de ligação logarítmica é aplicada a contagens cujo valor é zero. Para mais detalhes ver, por exemplo, McCullagh e Nelder (1989, Seção 2.5).

Exemplo 2.1 (continuação): Como visto anteriormente, no caso da distribuição de Poisson a função de variância é dada por $V\left(\mu_{i}\right)=\mu_{i}$, e o modelo de regressão de Poisson log-linear usual assume que $g\left(\mu_{i}\right)=\log \mu_{i}=\eta_{i}$ (ligação canônica). Assim temos que os pesos são as próprias médias, ou seja, $w_{i}=\mu_{i}$, e a variável dependente modificada será

$$
z_{i}=\log \mu_{i}+\frac{\left(y_{i}-\mu_{i}\right)}{\mu_{i}}
$$

\subsection{Função Desvio}

Define-se a função desvio com fator de escala como a distância entre o loga ritmo da função de verossimilhança do modelo saturado (com $n$ parâmetros) e do modelo sob investigação (com $p$ parâmetros, sendo $p<n$ ) avaliado na estimativa de máxima verossimilhança $\widehat{\beta}$, ou seja,

$$
S_{p}=\frac{D_{p}}{\phi},
$$


onde

$$
D_{p}=D_{p}(y ; \widehat{\mu})=2 \sum_{i=1}^{n}\left\{y_{i}\left[\widetilde{\theta}_{i}-\widehat{\theta}_{i}\right]-b\left(\widetilde{\theta}_{i}\right)+b\left(\widehat{\theta}_{i}\right)\right\},
$$

e com $\widetilde{\theta}_{i}=\theta\left(y_{i}\right)$ e $\widehat{\theta}_{i}=\theta\left(\widehat{\mu}_{i}\right)$ representando respectivamente as estimativas de máxima verossimilhança de $\theta$ para o modelo saturado ( $n$ parâmetros) e com $p$ parâmetros $(p<n)$. Uma medida usual de discrepância entre o modelo saturado e o modelo sob investigação é o desvio sem fator de escala, dado por $D_{p}$.

Um valor pequeno para a função desvio $D_{p}$, assim como para $S_{p}$, indicam que, para um número menor de parâmetros, obtém-se um ajuste tão bom quanto o ajuste com o modelo saturado. Em geral, nos MLG's (exceto no modelo normal linear) o desvio (com ou sem escala) não tem distribuição conhecida. Muitas vezes, se usa a aproximação $S_{p} \sim \chi_{n-p}^{2}$, mesmo que esta possa ser muito ruim. Esta aproximação é razoável quando $\phi$ é pequeno. Sendo assim, é comum se comparar o valor observado do desvio com a média da distribuição $\chi_{n-p}^{2}$, ou seja, $n-p$. Se tal valor for muito superior a $n-p$, entende-se que há evidências de que o modelo não fornece um bom ajuste.

Exemplo 2.1 (continuação): Como já mencionado, no caso da distribuição de Poisson tem-se $\phi=1, \theta_{i}=\log \mu_{i}$ e $b\left(\theta_{i}\right)=e^{\theta_{i}}$, o que implica em $\widetilde{\theta}_{i}=\log y_{i}$ e $\widehat{\theta}_{i}=\log \widehat{\mu}_{i}$, e assim,

$$
S_{p}=D_{p}=2 \sum_{i=1}^{n}\left\{y_{i} \log \left(\frac{y_{i}}{\widehat{\mu}_{i}}\right)-\left(y_{i}-\widehat{\mu}_{i}\right)\right\} .
$$

Se $y_{i}=0$ o $i$-ésimo termo de $D_{p}$ vale $2 \widehat{\mu}_{i}$. Como admitimos que o modelo inclui intercepto $\left(x_{i 1} \equiv 1\right)$, temos $\sum_{i=1}^{n}\left(y_{i}-\widehat{\mu}_{i}\right)=0$ e o desvio torna-se

$$
D_{p}=2 \sum_{i=1}^{n} y_{i} \log \left(\frac{y_{i}}{\widehat{\mu}_{i}}\right) \text {. }
$$

Vale destacar que para a distribuição normal, o desvio coincide com a soma de quadrados dos resíduos, ou seja, $D_{p}=\sum_{i=1}^{n}\left(y_{i}-\widehat{\mu}_{i}\right)^{2}$.

\subsection{Análise Inferencial}

Quando um modelo está sendo construído, é preciso ter o cuidado de verificar se este é adequado para descrever um conjunto de dados. O objetivo é que o modelo final seja o mais simples possível e que forneça um bom ajuste. 
Na tentativa de simplificar um modelo, é preciso verificar se algumas variáveis preditoras, $x_{q+1}, \ldots, x_{p-1}, x_{p}$ digamos, podem ser eliminadas do modelo. Em outras palavras é preciso testar

$$
\mathrm{H}_{0}: \beta_{q+1}=\beta_{q+2}=\ldots=\beta_{p}=0
$$

versus

$$
\mathrm{H}_{a} \text { : não todos os } \beta_{k}^{\prime} s \text { em } H_{0} \text { são iguais a zero. }
$$

Para isto é feita a comparação dos valores do desvio deste modelo com e sem as variáveis em questão. A diferença de desvios é denominada desvio parcial e é dada por

$$
D_{q}-D_{p}
$$

onde $D_{q}$ e $D_{p}$ são, respectivamente, os desvios apresentados em (2.7) com $q$ e $p$ variáveis explicativas. Tem-se assintoticamente

$$
S_{q}-S_{p}=\frac{D_{q}-D_{p}}{\phi} \sim \chi_{p-q}^{2} .
$$

Note que $S_{q}-S_{p}$ iguala-se à estatística da razão de verossimilhanças de $\mathrm{H}_{0}$ versus $\mathrm{H}_{a}$ se $\phi$ for conhecido.

A análise inferencial do modelo também pode ser facilmente desenvolvida através da distribuição assintótica do vetor $\widehat{\beta}$

$$
\widehat{\beta} \sim \mathrm{N}_{p}\left(\beta, I(\beta)^{-1}\right),
$$

onde $I(\beta)$ é a matriz de informação de Fisher para o parâmetro $\beta$ dada por

$$
I(\beta)=\phi X^{\top} W X
$$

Temos então as estimativas de máxima verossimilhança de $\beta$ e $I(\beta)$ dadas por $\widehat{\beta}$ e $\phi X^{\top} \widehat{W} X$ (se $\phi$ for desconhecido deve ser estimado a partir dos dados, por exemplo, pelo método de máxima verossimilhança). Seja $s^{2}\left(\widehat{\beta}_{i}\right)$ o elemento $(i, i)$ da matriz $I(\widehat{\beta})^{-1}$, o estimador da variância de $\widehat{\beta}_{i}$. Logo, para $n$ grande,

$$
\frac{\widehat{\beta}_{i}-\beta_{i}}{s\left(\widehat{\beta}_{i}\right)} \sim \mathrm{N}(0,1),
$$

aproximadamente. Testes de hipóteses baseados nas estatísticas de razão de verossimilhanças, escore e Wald são facilmente aplicáveis (Demétrio, 2001, Seção 2.10 e Paula, 2002, Cap. 2). Se $\phi$ for conhecido, como citado acima, o teste do desvio parcial e o teste da razão de verossimilhanças coincidem.

Existem também vários procedimentos para a seleção de modelos de regressão, embora nenhum deles seja consistente, ou seja, mesmo para amostras grandes 
$(n \rightarrow \infty)$ selecione com probabilidade um as variáveis explicativas com coeficiente de regressão não nulo. Os procedimentos mais conhecidos são forward, backward, stepwise e o método de Akaike $(A I C)$, além de outros métodos de computação intensiva. Paula (2002) descreve brevemente cada um deles.

Aqui vamos apresentar o método proposto por Akaike (1974), que é muito utilizado na seleção de MLG's e se diferencia dos demais por ser um processo de minimização que não envolve testes estatísticos. A idéia básica é selecionar um modelo que seja parcimonioso, ou seja, que esteja bem ajustado e tenha um número reduzido de parâmetros. Como o máximo do logaritmo da função de verossimilhança $l(\beta ; y)$ cresce com o aumento do número de parâmetros do modelo, uma proposta razoável seria encontrar o modelo com menor valor para a função

$$
A I C=-2 l(\widehat{\beta} ; y)+2 p
$$

onde $p$ denota o número de parâmetros.

Exemplo 2.1 (continuação): Para a distribuição de Poisson, temos

$$
A I C=-2 \sum_{i=1}^{n}\left(y_{i} \log \widehat{\mu}_{i}-\widehat{\mu}_{i}-\log y_{i} !\right)+2 p .
$$

Independentemente do modelo em estudo, inferências sobre a resposta média também são necessárias, pois muitas vezes é preciso estimar a média $\mu_{h}$ para um conjunto particular de valores das variáveis explicativas $x_{h}$. A estimativa pontual da média é dada por $\widehat{\mu}_{h}=g^{-1}\left(x_{h}^{\top} \widehat{\beta}\right)=g^{-1}\left(\widehat{\eta}_{h}\right)$, e um intervalo de confiança para $\mu_{h}$ é obtido em dois estágios: primeiro é necessário calcular o intervalo de confiança para o preditor linear $\eta_{h}$, e depois fazer a conversão desse intervalo para um intervalo de confiança para a resposta média $\mu_{h}$ através da função de ligação utilizada $g(\cdot)$.

\subsection{Técnicas de Diagnóstico}

Após ajustar um modelo, é indispensável verificar se o mesmo é adequado para representar os dados em estudo. Técnicas de diagnóstico são de grande relevância para detectar problemas em modelos de regressão, tais como, inadequação do modelo utilizado e a presença de outliers e observações influentes. 
Como mencionamos na Seção 2.3, uma maneira informal de examinar o ajuste do modelo é comparar o valor observado do desvio dado em (2.6) com $n-p$. Se esse valor for muito superior a $n-p$, o ajuste do modelo é considerado inadequado.

Uma simples técnica para examinar o ajuste do modelo é a construção de um gráfico dos valores observados $\left(y_{1}, \ldots, y_{n}\right)$ versus os valores ajustados $\left(\widehat{\mu}_{1}, \ldots, \widehat{\mu}_{n}\right)$. Se o ajuste for coerente, espera-se que os pontos estejam próximos da reta $x=y$.

Uma outra maneira de avaliar um modelo é através das medidas que comparam os valores observados com os valores preditos pelo modelo, denominados resíduos. A análise de resíduos é feita através de métodos gráficos.

Um tipo de resíduo muito utilizado nos MLG's é o "resíduo do desvio" (ou "componente do desvio"). O resíduo do desvio para o caso $i$ é a contribuição da $i$-ésima observação na soma do desvio do modelo, e é definido como

$$
\operatorname{dev}_{i}=\operatorname{sinal}\left(y_{i}-\widehat{\mu}_{i}\right)\left\{2 y_{i}\left[\widetilde{\theta}_{i}-\widehat{\theta}_{i}\right]-2 b\left(\widetilde{\theta}_{i}\right)+2 b\left(\widehat{\theta}_{i}\right)\right\}^{1 / 2}
$$

Uma importante propriedade do resíduo do desvio é que

$$
\sum_{i=1}^{n}\left(\operatorname{dev}_{i}\right)^{2}=D_{p}
$$

Os resíduos de Pearson são muito utilizados para identificação de possíveis outliers e são definidos como

$$
r_{i}=\frac{y_{i}-\widehat{\mu}_{i}}{\sqrt{\widehat{\operatorname{Var}\left(y_{i}\right)}}}=\frac{y_{i}-\widehat{\mu}_{i}}{\sqrt{\phi V\left(\widehat{\mu}_{i}\right)}},
$$

onde $\widehat{\mu}_{i}$ e $\widehat{\operatorname{Var}\left(y_{i}\right)}$ são estimativas de máxima verossimilhança da média e da variância de $y_{i}$, obtidas com o ajuste do modelo em estudo.

Outros tipos de resíduos utilizados nos MLG's são os resíduos do desvio padronizados, resíduos ordinários, resíduos de Anscombe, entre outros. Para um estudo mais detalhado podem ser consultados Paula (2002, Seção 2.9), Demétrio (2001, Seção 3.4) e McCullagh e Nelder (1989, Capítulo 10) por exemplo.

Uma sugestão para identificação de possíveis outliers e verificação da suposição de independência entre as observações são os gráfico dos resíduos componentes do desvio $\left(d e v_{i}\right)$ e resíduos de Pearson $\left(r_{i}\right)$ contra alguma função dos dados, como por exemplo, as estimativas dos preditores lineares $\widehat{\eta}_{i}=x_{i}^{\top} \widehat{\beta}$ ou mesmo seu índice $i$. Espera-se uma distribuição aleatória dos resíduos em torno do eixo horizontal. Os pontos (resídūos) afastados dos demais devem ser retirados do modelo corrente para uma nova avaliação e se houver redução considerável no desvio do modelo, estes pontos devem ser avaliados com especial atenção. 
O $i$-ésimo elemento da diagonal da matriz $H=W^{1 / 2} X\left(X^{\top} W X\right)^{-1} X^{\top} W^{-1 / 2}$ pode ser visto como uma medida de alavanca $h_{i}$ (leverage) da observação correspondente. Um gráfico das estimativas de $h_{1}, \ldots, h_{n}$ contra os valores ajustados $\widehat{\mu}_{1}, \ldots, \widehat{\mu}_{n}$ pode ser útil para detectar observações com altos valores de alavanca. Essas observações podem ser potencialmente influentes no ajuste do modelo. A distância de Cook é freqüentemente utilizada como uma medida de influência (Cook, 1986), pois considera o efeito da $i$-ésima observação sobre todos os valores ajustados pelo modelo. Sua definição é dada por $2\left\{l(\widehat{\beta})-l\left(\widehat{\beta}_{(i)}\right)\right\}$, onde $l(\widehat{\beta})$ é o logaritmo da função de verossimilhança avaliado em $\widehat{\beta}$ e $\widehat{\beta}_{(i)}$ é a estimativa de $\beta$ obtida com a retirada da $i$-ésima observação. No caso dos MLG's, não é possível escrever essa medida em uma forma fechada, mas esta pode ser aproximada por $r_{i}^{2} h_{i} /\left(1-h_{i}\right)^{2}$. Paula (2002, Capítulo 2) apresenta um estudo mais abrangente sobre pontos de alavanca e influência nos MLG's.

Sempre que os dados são obtidos em uma seqüência de tempo, uma maneira informal de avaliar se a suposição de que, dados os valores das covariadas, as observações são independentes é através do cálculo das correlações entre os resíduos $r_{i}$ e $r_{i-L}$ para $L=1,2,3, \ldots$, denominadas autocorrelações de defasagem (lag) $L$ (Morettin e Toloi, 1985). A construção de um gráfico com as autocorrelações para diferentes defasagens (função de autocorrelação) é muito útil na análise de diagnóstico, onde espera-se que as autocorrelações estejam próximas de zero caso a independência entre as observações esteja satisfeita. Se a correlação entre os resíduos mais próximos no tempo é maior do que entre os resíduos mais distantes, dizemos que existe autocorrelação serial. Isto não acarretará viés nos coeficientes do modelo estimados, mas os erros padrão poderão ser sub ou superestimados. A adoção do modelo de Zeger (Zeger e Liang, 1986) é uma alternativa para a análise de dados correlacionados por apresentar uma estrutura de dependência incorporada ao modelo, cujos parâmetros são estimados através de equações de estimação generalizadas.

Exemplo 2.1 (continuação): No caso da distribuição de Poisson, o resíduo do desvio é dado por

$$
\operatorname{dev}_{i}=\operatorname{sinal}\left(y_{i}-\widehat{\mu}_{i}\right)\left\{2\left[y_{i} \log \left(\frac{y_{i}}{\widehat{\mu}_{i}}\right)-\left(y_{i}-\widehat{\mu}_{i}\right)\right]\right\}^{1 / 2},
$$

e o resíduo de Pearson por

$$
r_{i}=\frac{y_{i}-\widehat{\mu}_{i}}{\sqrt{\widehat{\mu}_{i}}}
$$

Para o modelo de regressão log-linear de Poisson temos $h_{i}=\mu_{i} x_{i}^{\top}\left(X^{\top} W X\right)^{-1} x_{i}$. 
Como nos MLG's a distribuição dos resíduos não é conhecida, gráficos de probabilidade meio-normal com envelope simulado são uma ferramenta útil para propósitos de diagnóstico (Atkinson, 1985, Neter et al., 1996, Seção 14.6). A idéia é acrescentar aos gráficos de probabilidade meio-normal um envelope simulado que pode ser utilizado para decidir se as respostas observadas são consistentes com o modelo ajustado. O resíduo do desvio, como definido em (2.10), é bastante utilizado na construção de gráficos de probabilidade meio-normal com envelope simulado ${ }^{1}$. Se o modelo for adequado espera-se que a grande maioria dos resíduos esteja dentro do envelope simulado. Esses gráficos são úteis não só para avaliar a adequação do preditor linear, da função de ligação, da distribuição escolhida para a variável resposta do modelo em estudo, como também para identificar outliers, caso os resíduos com maiores valores absolutos excedam os limites do envelope simulado.

Gráficos de probabilidade meio-normal com envelope simulado são construídos da seguinte forma:

1. ajuste o modelo e gere uma amostra simulada de $n$ observações independentes utilizando o modelo ajustado como se fosse o modelo verdadeiro;

2. ajuste o modelo para a nova amostra, e calcule os valores absolutos ordenados da medida de diagnóstico de interesse;

3. repita os passos acima 18 vezes;

4. considere os $n$ conjuntos de 19 estatísticas ordenadas; para cada conjunto, calcule as respectivas médias, valores mínimos e máximos;

5. faça o gráfico desses valores e da medida de diagnóstico ordenada da amostra original contra os escores meio-normais $\Phi^{-1}((i+n-1 / 8) /(2 n+1 / 2))$, onde $\Phi($.$) é a função distribuição acumulada normal padrão.$

Os valores mínimos e máximos das 19 estatísticas ordenadas constituem o envelope. Grandes desvios dos pontos em torno da média dos valores simulados ou a ocorrência de pontos próximos dos limites ou fora do envelope simulado são indicadores de que o modelo não é apropriado.

Exemplo 2.1 (continuação): Para a análise da qualidade do ajuste de um modelo de regressẩo de Poisson o gráfico de probabilidade meio-normal com en-

\footnotetext{
${ }^{1}$ Este gráfico também pode ser construído para os resíduos de Pearson, ou qualquer outra medida de diagnóstico.
} 
velope simulado dos resíduos do desvio deve ser construído como descrito acima, onde o passo (1) neste caso é dado por

1. ajuste o modelo de regressão de Poisson e gere uma amostra simulada $y_{1}^{*}, \ldots, y_{n}^{*}$ de $n$ observações independentes, onde $Y_{i}^{*} \sim \operatorname{Poisson}\left(\widehat{\mu}_{i}\right)$.

Um gráfico de probabilidade meio-normal com envelope simulado dos resíduos de Pearson ou resíduos do desvio do ajuste de um modelo de Poisson pode ser utilizado como uma verificação informal de superdispersão. Uma parcela considerável de pontos acima do envelope simulado indica que a variabilidade dos resíduos é maior que a esperada, e portanto um modelo de superdispersão pode ser mais apropriado para o conjunto de dados em estudo.

Estas e outras técnicas de diagnóstico para MLG's estão apresentadas detalhadamente em McCullagh e Nelder (1989, Cap. 10), Demétrio (2001, Cap. 3) e Paula (2002, Seção 2.9), por exemplo.

\subsection{Modelo Binomial Negativo}

Svetliza (2002) apresenta um breve histórico da distribuição binomial negativa, onde descreve que suas formas especiais foram discutidas preliminarmente por Pascal em 1679. A Associação Brasileira de Estatística (ver http://www.ime .usp. $\mathrm{br} / \sim a b e / c r o n o l o g i a j a n e i r o 02$.pdf) apresenta uma cronologia de alguns conceitos e fatos importantes da estatística que aponta a origem da distribuição binomial negativa no ano de 1713, dada por Montmort. Recentemente, a distribuição binomial negativa tem sido muito utilizada como uma primeira alternativa à de Poisson em caso de superdispersão. Como já comentado no Capítulo 1, uma consequiência importante de se ignorar a superdispersão é que os erros padrão obtidos através do modelo de regressão de Poisson são incorretos e subestimam a variabilidade dos estimadores de seus parâmetros. Assim, podemos dizer que a distribuição binomial negativa é útil para se definir um modelo de regressão para contagens superdispersas.

Se a variável aleatória $Y$ tem distribuição binomial negativa com parâmetros $\mu>0$ e $k>0$, sua função de probabilidade é dada por

$$
f_{Y}(y ; \mu, k)=\frac{\Gamma(y+k)}{\Gamma(y+1) \Gamma(k)}\left(\frac{k}{k+\mu}\right)^{k}\left(\frac{\mu}{k+\mu}\right)^{y}, y=0,1, \ldots,
$$

onde $\Gamma($.) é a função gama. Nesse caso, escrevemos $Y \sim \mathrm{BN}(k, \mu)$. 
Uma possível motivação para assumir que as contagens observadas procedem de uma distribuição binomial negativa é que (2.12) aparece como a distribuição marginal de $Y$ quando assumimos que $Y \mid Z \sim \operatorname{Poisson}(Z)$ e $Z \sim \operatorname{Gama}(\mu, k)$, com $\mu>0, k>0$ e $k$ independente de $\mu$. A função densidade de probabilidade da distribuição gama nesse caso assume a forma

$$
f_{Z}(z ; \mu, k)=\frac{1}{\Gamma(k)}\left(\frac{z k}{\mu}\right)^{k} \exp \left\{-\left(\frac{k z}{\mu}\right)\right\}, \quad z>0 .
$$

Assim, a esperança e variância de $Y$ são dadas por

$$
\mathrm{E}(Y)=\mathrm{E}[\mathrm{E}(Y \mid Z)]=\mu
$$

$\mathrm{e}$

$$
\operatorname{Var}(Y)=\mathrm{E}[\operatorname{Var}(Y \mid Z)]+\operatorname{Var}[\mathrm{E}(Y \mid Z)]=\mu+\frac{\mu^{2}}{k}
$$

Note-se, portanto, que $\operatorname{Var}(Y)>\mathrm{E}(Y)$ (para mais detalhes ver David, 2000).

Quando $k$ é inteiro a função de probabilidade da distribuição binomial negativa pode ser escrita como

$$
f_{Y}(y ; \mu, k)=\left(\begin{array}{c}
y+k-1 \\
y
\end{array}\right)\left(\frac{k}{k+\mu}\right)^{k}\left(\frac{\mu}{k+\mu}\right)^{y}, \quad y=0,1, \ldots
$$

Para mais detalhes sobre a distribuição de probabilidade binomial negativa ver, por exemplo, Johnson, Kotz e Kemp (1993).

A distribuição binomial negativa com o parâmetro $k$ conhecido faz parte da familia exponencial, pois sua função de probabilidade é da forma (2.1) com

$$
\begin{gathered}
\phi=1, \quad \theta=\log \frac{\mu}{\mu+k}, \quad \mu=\frac{k e^{\theta}}{1-e^{\theta}}, \\
b(\theta)=-k \log \left(1-e^{\theta}\right), \quad c(y, \phi)=\log \frac{\Gamma\left(y_{i}+k\right)}{\Gamma\left(y_{i}+1\right) \Gamma(k)},
\end{gathered}
$$

e função de variância

$$
V(\mu)=\mu+\frac{\mu^{2}}{k}
$$

O modelo de regressão binomial negativo assume que as variáveis aleatórias independentes $Y_{1}, \ldots, Y_{n}$ têm distribuição binomial negativa com médias $\mu_{1}, \ldots, \mu_{n}$ respectivamente, e um parâmetro comum $k$, e que as médias estão relacionadas com as covariadas $x_{1}, \ldots, x_{n}$ através da função de ligação (2.2), lembrando que $x_{i}=\left(1, x_{i 2}, \ldots, x_{i p}\right)$. Para $k$ conhecido, como visto na Seção 2.1, este modelo pertence à classe dos MLG's. No entanto, a suposição de que o parâmetro de 
dispersão $k$ da distribuição binomial negativa é conhecido é geralmente irreal e métodos adequados de inferência nesse modelo serão desenvolvidos a seguir.

A função de verossimilhança de $\left(\beta^{\top}, k\right)$ para a amostra $y=\left(y_{1}, \ldots, y_{n}\right)$ é dada por

$$
L(\beta, k ; y)=\prod_{i=1}^{n} \frac{\Gamma\left(y_{i}+k\right)}{\Gamma\left(y_{i}+1\right) \Gamma(k)}\left(\frac{k}{k+\mu_{i}}\right)^{k}\left(\frac{\mu_{i}}{k+\mu_{i}}\right)^{y_{i}}
$$

com $\mu_{i}=g^{-1}\left(x_{i}^{\top} \beta\right)$, e logo, o logaritmo da função de verossimilhança é

$$
\begin{aligned}
l(\beta, k ; y)= & \log L(\beta, k ; y)= \\
= & \sum_{i=1}^{n}\left\{y_{i} \log \mu_{i}+k \log k-\left(k+y_{i}\right) \log \left(k+\mu_{i}\right)+\log \Gamma\left(y_{i}+k\right)\right. \\
& \left.-\log \Gamma(k)-\log y_{i} !\right\} .
\end{aligned}
$$

As derivadas de primeira e segunda ordem de $l(\beta, k ; y)$ são dadas por

$$
\begin{gathered}
\frac{\partial l}{\partial \beta_{r}}=\sum_{i=1}^{n}\left\{\frac{y_{i}}{\mu_{i}}-\frac{k+y_{i}}{k+\mu_{i}}\right\} \frac{\partial \mu_{i}}{\partial \beta_{r}}=\sum_{i=1}^{n} \frac{\left(y_{i}-\mu_{i}\right)}{V\left(\mu_{i}\right) g^{\prime}\left(\mu_{i}\right)} x_{i r} \\
\frac{\partial^{2} l}{\partial \beta_{r} \partial \beta_{s}}=\sum_{i=1}^{n}\left\{\frac{-V\left(\mu_{i}\right) g^{\prime}\left(\mu_{i}\right)-\left(y_{i}-\mu_{i}\right)\left[V^{\prime}\left(\mu_{i}\right) g^{\prime}\left(\mu_{i}\right)+V\left(\mu_{i}\right) g^{\prime \prime}\left(\mu_{i}\right)\right]}{\left[V\left(\mu_{i}\right) g^{\prime}\left(\mu_{i}\right)\right]^{2}}\right\} \frac{x_{i s} x_{i r}}{g^{\prime}\left(\mu_{i}\right)} \\
r, s=1, \ldots, p, \mathrm{e} \\
\frac{\partial l}{\partial \beta_{r} \partial k}=\sum_{i=1}^{n} \frac{\left(y_{i}-\mu_{i}\right)}{\left(k+\mu_{i}\right)^{2}} \frac{1}{g^{\prime}\left(\mu_{i}\right)} x_{i r} \\
\frac{\partial k}{\partial k}\left\{\psi\left(y_{i}+k\right)-\psi(k)+\log \left(\frac{k}{\mu_{i}+k}\right)-\frac{k+y_{i}}{k+\mu_{i}}+1\right\} \\
\frac{\partial^{2} l}{\partial k^{2}}=\sum_{i=1}^{n}\left\{\psi^{\prime}\left(y_{i}+k\right)-\psi^{\prime}(k)+\frac{y_{i}-2 \mu_{i}-k}{\left(k+\mu_{i}\right)^{2}}+\frac{1}{k}\right\}
\end{gathered}
$$

onde $V\left(\mu_{i}\right)=\mu_{i}+\mu_{i}^{2} / k$ e $\psi(\cdot)=\Gamma^{\prime}(\cdot) / \Gamma(\cdot)$ representa a função digama. Assim, temos que a função escore $U(\beta, k)=\left(\partial l / \partial \beta_{1}, \ldots, \partial l / \partial \beta_{p}, \partial l / \partial k\right)^{\top}$ tem elementos dados por (2.14) e (2.15).

A matriz de informação de Fisher $I(\beta, k)$ é dada por

$$
I(\beta, k)=\left[i_{r s}(\beta, k)\right] \quad r=1, \ldots, p+1 ; s=1, \ldots, p+1
$$


com

$$
\begin{gathered}
i_{r s}(\beta, k)=-\mathrm{E}\left\{\frac{\partial^{2} l}{\partial \beta_{r} \partial \beta_{s}}\right\}=\sum_{i=1}^{n} \frac{x_{i r} x_{i s}}{V\left(\mu_{i}\right)\left[g^{\prime}\left(\mu_{i}\right)\right]^{2}}, \quad r, s=1, \ldots, p, \\
i_{r, p+1}(\beta, k)=i_{p+1, r}(\beta, k)=-\mathrm{E}\left\{\frac{\partial^{2} l}{\partial \beta_{r} \partial k}\right\}=0, \quad r=1, \ldots, p, \\
i_{p+1, p+1}(\beta, k)=-\mathrm{E}\left\{\frac{\partial^{2} l}{\partial k^{2}}\right\}=\sum_{i=1}^{n}\left\{-\mathrm{E}\left(\psi^{\prime}\left(Y_{i}+k\right)\right)+\psi^{\prime}(k)+\frac{1}{\mu_{i}+k}-\frac{1}{k}\right\} .
\end{gathered}
$$

Ou, na forma matricial,

$$
I(\beta, k)=\left[\begin{array}{cc}
X^{\top} W X & 0 \\
0^{\top} & i_{p+1, p+1}
\end{array}\right]
$$

onde $X^{\top}=\left(x_{1}, \ldots, x_{n}\right), 0$ representa o vetor de zeros de dimensão $p \times 1 \mathrm{e}$ $W=\operatorname{diag}\left(w_{1}, \ldots, w_{n}\right)$ é a matriz de pesos apresentada em (2.5) com

$$
w_{i}=\left(\frac{d \mu_{i}}{d \eta_{i}}\right)^{2} \frac{1}{\mu_{i}+\mu_{i}^{2} / k} .
$$

Observe que $I(\beta, k)$ é uma matriz bloco diagonal e, portanto, $\beta$ e $k$ são parâmetros ortogonais. A matriz de covariância assintótica do estimador de máxima verossimilhança de $\beta$ é $\left(X^{\top} W X\right)^{-1}$ se $k$ é conhecido ou mesmo se é estimado a partir dos dados.

O modelo de regressão binomial negativo mais usual assume a função de ligação logarítmica $g(\mu)=\log (\mu)=\eta$, e é mais conhecido como modelo de regresão log-linear binomial negativo, e nesse caso temos

$$
W=\operatorname{diag}\left(w_{1}, \ldots, w_{n}\right) \quad \operatorname{com} w_{i}=\frac{k \mu_{i}}{k+\mu_{i}} .
$$

A forma bloco diagonal da matriz de informação $I(\beta, k)$ permite que as estimativas de máxima verossimilhança para $\beta$ e $k$ sejam obtidas pela aproximação de Gauss-Seidel, da seguinte forma:

(i) para um valor fixo de $k$, obtenha a solução de $\partial l / \partial \beta_{r}=0$, para $r=1, \ldots, p$, aplicando o algoritmo dos mínimos quadrados reponderados ${ }^{2}$ em (2.14) com função de variância $\mathrm{V}(\mu)=\mu+\mu^{2} / k$ (ver McCullagh e Nelder, 1989);

\footnotetext{
${ }^{2}$ Método padrão para o ajuste de MLG's, como descrito na Seção 2.2.
} 
(ii) Para um valor fixo de $\beta$, e logo de $\mu$ (obtido no passo anterior), obtenha a solução de $\partial l / \partial k=0$ aplicando o método iterativo de Newton-Raphson em (2.15). Conforme descrito em Rustagi (1994), o algoritmo de NewtonRaphson consiste em solucionar iterativamente uma equação do tipo

$$
k^{(m)}=k^{(m-1)}-\frac{f\left(k^{(m-1)}\right)}{f^{\prime}\left(k^{(m-1)}\right)},
$$

onde $k^{(m)}$ e $k^{(m-1)}$ são estimativas de $k$ na $m$-ésima e (m-1)-ésima iteração, respectivamente. Neste caso temos

$$
f(k)=\frac{\partial l}{\partial k} \quad \text { e } \quad f^{\prime}(k)=\frac{\partial^{2} l}{\partial k^{2}},
$$

descritos em (2.15) e (2.16), respectivamente. Podemos então escrever o processo (2.20) como

$$
k^{(m)}=k^{(m-1)}-\left.\left(\frac{\partial l}{\partial k} / \frac{\partial^{2} l}{\partial k^{2}}\right)\right|_{k=k^{(m-1)}} .
$$

(iii) repita os passos (i) e (ii) alternando a estimação de $\beta$ e $k$ até que este processo convirja, obtendo assim as estimativas de máxima verossimilhança $\widehat{\beta}$ e $\widehat{k}$.

Um valor inicial para $k$ é

$$
k^{(0)}=\frac{\sum_{i=1}^{n} \widehat{\mu}_{i}\left(1-c_{i} \widehat{\mu}_{i}\right)}{\sum_{i=1}^{n} \frac{\left(y_{i}-\hat{\widehat{A}}_{i}\right)^{2}}{\widehat{\mu}_{i}}-(n-p)},
$$

onde aqui $\widehat{\mu}_{i}$ é o estimador de máxima verossimilhança de $\mu_{i}$ obtido pelo modelo de regressão de Poisson e $c_{i}=x_{i}^{\top}\left(X^{\top} \widehat{W} X\right)^{-1} x_{i}$, com $\widehat{W}=W(\widehat{\mu})$, que é a estimativa da variância assintótica de $\widehat{\eta}_{i}=x_{i}^{\top} \widehat{\beta}$. A idéia vem da comparação da estatística de Pearson do ajuste do modelo de Poisson, isto é, $X^{2}=\sum_{i=1}^{n}\left(y_{i}-\widehat{\mu}_{i}\right)^{2} / \widehat{\mu}_{i}$ com o valor esperado sob o modelo binomial negativo (ver Breslow, 1984, para detalhes).

O software estatístico S-Plus 4.5 possui a função glm.nb na biblioteca $M A S S$ (Venables e Ripley, 1997) que ajusta este modelo com distribuição binomial negativa fornecendo as estimativas de máxima verossimilhança conjunta $\widehat{\beta}$ e $\widehat{k}$ e respectivos desvios padrão. Ver Hardin e Hilbe (2001, Capítulo 13) e Pedan (2001) para detalhes sobre o ajuste desse modelo usando o STATA e o SAS respectivamente.

Assumindo $k>0$, em grandes amostras podemos construir intervalos de confiança e testes de hipóteses admitindo que $\left(\widehat{\beta}^{\top}-\beta^{\top}, k-\widehat{k}\right)^{\top}$ assume distribuição normal com média zero e matriz de variância-covariância

$$
s^{2}\{\widehat{\beta}, \widehat{k}\}=I(\beta, k)^{-1},
$$


onde $I(\beta, k)$ é a matriz de informação de Fisher dada em (2.18).

Logo, quando a amostra é grande, inferências sobre os coeficientes do modelo binomial negativo podem ser baseadas nas seguintes aproximações

$$
\frac{\widehat{\beta}_{r}-\beta_{r}}{\sqrt{\widehat{s}_{r r}^{2}\{\hat{\beta}, \widehat{k}\}}} \sim N(0,1), \quad r=1, \ldots, p,
$$

$\mathrm{e}$

$$
\frac{\widehat{k}-k}{\sqrt{\widehat{s}_{p+1, p+1}^{2}\{\widehat{\beta}, \widehat{k}\}}} \sim N(0,1)
$$

onde $\widehat{s}_{r r}^{2}\{\widehat{\beta}, \widehat{k}\}$ é o elemento $(r, r)$ da matriz $s^{2}\{\widehat{\beta}, \widehat{k}\}$, dada em (2.21), avaliada em $(\widehat{\beta}, \widehat{k})$. Observe que $\widehat{s}_{p+1, p+1}^{2}\{\widehat{\beta}, \widehat{k}\}$ é o inverso do elemento $(p+1, p+1)$ da matriz $s^{2}\{\widehat{\beta}, \widehat{k}\}$, dada em (2.21), avaliada em $(\widehat{\beta}, \widehat{k})$.

A partir deste resultado, obtém-se diretamente um intervalo de confiança para $\beta_{r}$ com coeficiente de confiança aproximado $\gamma=1-\alpha$ e limites

$$
\widehat{\beta}_{r} \mp z_{\alpha / 2} \sqrt{\widehat{s}_{r r}^{2}\{\hat{\beta}, \widehat{k}\}}
$$

onde $z_{\alpha}$ é o quantil de ordem $1-\alpha$ da distribuição normal padrão. Um intervalo de confiança com coeficiente de confiança aproximado $\gamma=1-\alpha$ para $k$ tem limites

$$
\widehat{k} \mp z_{\alpha / 2} \sqrt{\widehat{s}_{p+1, p+1}^{2}\{\widehat{\beta}, \widehat{k}\}} .
$$

O procedimento de Bonferroni fornece um intervalo de confiança simultâneo para vários parâmetros do modelo com distribuição binomial negativa. Se $g$ parâmetros são estimados com coeficiente de confiança conjunto (maior ou igual a) $\gamma=1-\alpha$, o intervalo de confiança para $\beta_{r}$ terá limites dados por

$$
\widehat{\beta}_{r} \mp z_{\alpha /(2 g)} \sqrt{\widehat{s}_{r r}^{2}\{\widehat{\beta}, \widehat{k}\}} \text {. }
$$

Baseado no resultado (2.22), um teste para grandes amostras com um único parâmetro de interesse $\beta_{r}$ pode ser construído para as seguintes hipóteses

$$
\mathrm{H}_{0}: \beta_{r}=0 \text { e } \mathrm{H}_{a}: \beta_{r} \neq 0 \text {. }
$$

Uma estatística apropriada para o teste é dada por

$$
z^{*}=\frac{\widehat{\beta_{r}}}{\sqrt{\widehat{s}_{r r}^{2}\{\widehat{\beta}, \widehat{k}\}}},
$$


e tem, aproximadamente, distribuição $N(0,1)$ sob $\mathrm{H}_{0}$. O teste rejeita $\mathrm{H}_{0}$ a um nível de significância $\alpha$ se e somente se $\left|z^{*}\right|>z_{\alpha / 2}$. Da mesma forma, podemos também construir testes unilaterais.

Outro procedimento bastante utilizado para o ajuste do modelo binomial negativo é obter uma estimativa, $\widetilde{k}$, para $k$ pelo método dos momentos, e ajustar um modelo linear generalizado com distribuição binomial negativa e $k=\widetilde{k}$, pois como já descrito no início dessa seção, para valores fixos (conhecidos) de $k$, esta distribuição faz parte da família exponencial.

Breslow (1984) sugere que a estimativa de $k$ seja obtida solucionando a seguinte equação

$$
\sum_{i=1}^{n} \frac{\left(y_{i}-\widetilde{\mu}_{i}\right)^{2}}{V\left(\widetilde{\mu}_{i}\right)}=\sum_{i=1}^{n} \frac{\left(y_{i}-\widetilde{\mu}_{i}\right)^{2}}{\left(\widetilde{\mu}_{i}+\frac{\widetilde{\mu}_{i}^{2}}{k}\right)}=n-p,
$$

onde $\widetilde{\mu}_{i}$ 's são os valores estimados pelo modelo de regressão de Poisson $(k=\infty)$, $n$ o tamanho da amostra e $p$ o número de variáveis explicativas. Para obtenção da estimativa $\widetilde{k}$, o método de Newton-Raphson (apresentado em (2.20)) pode ser aplicado em (2.23), com

$$
f(k)=\sum_{i=1}^{n} \frac{\left(y_{i}-\widetilde{\mu}_{i}\right)^{2}}{\left(\widetilde{\mu}_{i}+\frac{\widetilde{\mu}_{i}^{2}}{k}\right)}-n+p \text { e } f^{\prime}(k)=\sum_{i=1}^{n}\left(\frac{y_{i}-\widetilde{\mu}_{i}}{k+\widetilde{\mu}_{i}}\right)^{2},
$$

que leva ao seguinte processo iterativo

$$
k^{(m)}=k^{(m-1)}-\left.\frac{f(k)}{f^{\prime}(k)}\right|_{k=k^{(m-1)}} .
$$

(Se não houver solução tal que $\widetilde{k}>0$, admitir $\widetilde{k}=\infty$ ).

Uma vez obtida a estimativa $\widetilde{k}$, o ajuste e características do modelo binomial negativo se encaixam perfeitamente nos resultados dos MLG's apresentados nas Seções 2.1 a 2.5. Venables e Ripley (1997) apresentam a função neg.bin(k) que ajusta o modelo linear generalizado com distribuição binomial negativa ( $k$ conhecido).

Podemos dizer então que, para amostras grandes, o estimador de máxima verossimilhança $\widehat{\beta}$ de $\beta$ apresenta distribuição aproximadamente normal com média zero e matriz de variância-covariância $X^{\top} \widehat{W} X$, apresentada em (2.17), que corresponde à matriz de informação de Fisher avaliada em $\widehat{\beta}, \operatorname{com} k=\widetilde{k}$. A partir deste resultado, obtém-se diretamente intervalos de confiança e testes de hipóteses para $\beta$, como já descrito anteriormente. Se o método dos momentos é utilizado para obtenção de $k$, na prática a variabilidade de $k$ é ignorada. Moore (1986) e Lawless (1987) apresentam alguns resultados distribucionais complexos para $\widetilde{k}$, que não serão considerados neste estudo. 
A utilização do método dos momentos para a obtenção de uma estimativa para $k$ é mais robusta que o método de máxima verossimilhança, no entanto é menos eficiente se o modelo binomial negativo estiver correto. Lawless (1987) examina este tópico com detalhes.

A partir dos valores ajustados $\widehat{\mu_{i}}$ 's pelo modelo binomial negativo com função de ligação $g\left(\mu_{i}\right)=\eta_{i}=x_{i}^{\top} \widehat{\beta}$, pode-se facilmente calcular o desvio do modelo, definido em (2.6), que é dado por

$$
S_{p}=D_{p}=2 \sum_{i=1}^{n}\left\{y_{i}\left[\log \left(\frac{y_{i}}{y_{i}+k}\right)-\log \left(\frac{\widehat{\mu_{i}}}{\widehat{\mu}_{i}+k}\right)\right]+k \log \left(\frac{\widehat{\mu_{i}}+k}{y_{i}+k}\right)\right\} .
$$

Se $k$ é conhecido, assim como apresentado na Seção 2.4, reduções no modelo podem ser baseadas no desvio parcial. Seja $\mathrm{H}_{0}: \beta_{q+1}=\beta_{q+2}=\ldots=\beta_{p}=0 \mathrm{a}$ hipótese nula a ser testada contra a hipótese alternativa bilateral. Sob a hipótese nula, o desvio parcial tem distribuição assintótica qui-quadrado com $p-q$ graus de liberdade. Esse teste equivale ao teste da razão de verossimilhanças. Se $k$ é desconhecido, reduções no modelo podem ser baseadas no teste $\mathrm{F}$ aproximado

$$
F=\frac{\left(D_{q}-D_{p}\right) /(p-q)}{D_{p} /(n-p)} \sim F_{p-q, n-p},
$$

lembrando que $p$ e $q$ representam o número de covariadas em cada modelo $(p>q)$, e que os desvios $D_{p}$ e $D_{q}$ devem ser calculados considerando a estimativa de $k$ sob o modelo irrestrito (com $p$ covariadas).

Da mesma forma, se $k$ for conhecido, podemos obter facilmente a função de Akaike $(A I C)$ para o modelo binomial negativo, que pela sua definição apresentada em (2.9), é dada por

$$
\begin{aligned}
A I C= & -2 \sum_{i=1}^{n}\left\{y_{i} \log \widehat{\mu}_{i}+k \log k-\left(k+y_{i}\right) \log \left(k+\widehat{\mu}_{i}\right)+\log \Gamma\left(y_{i}+k\right)\right. \\
& \left.-\log \Gamma(k)-\log y_{i} !\right\}+2 p,
\end{aligned}
$$

$\operatorname{com} \widehat{\mu}_{i}=g^{-1}\left(x_{i}^{\top} \widehat{\beta}\right)$.

Independentemente do método de ajuste adotado, inferências sobre a resposta média também são necessárias, pois muitas vezes é preciso estimar a média $\mu$ para um conjunto particular de valores das variáveis explicativas. Utilizaremos como exemplo para o estudo da análise inferencial da resposta média o modelo log-linear binomial negativo, onde temos que $\mu=\exp \left(x^{\top} \beta\right)$.

$O$ vetor de valores das variáveis preditoras para o qual $\mu$ será estimado é denotado por $x_{h}=\left(1, x_{h 2}, \ldots, x_{h p}\right)^{\top}$, e a resposta média de interesse será $\mu_{h}=$ $\exp \left(x_{h}^{\top} \beta\right)$. A estimativa pontual de $\mu_{h}$, denotada por $\widehat{\mu}_{h}$ é dada por

$$
\widehat{\mu}_{h}=\exp \left(x_{h}^{\top} \widehat{\beta}\right) \text {. }
$$


Um intervalo de confiança para $\mu_{h}$ é obtido em dois estágios:

1) Primeiro, é necessário calcular o intervalo de confiança para o preditor linear $\eta_{h}$. A estimativa pontual de $\eta_{h}=x_{h}^{\top} \beta$ é $\widehat{\eta}_{h}=x_{h}^{\top} \widehat{\beta}$ e então a estimativa da variância de $\widehat{\eta}_{h}$ é dada por

$$
\widehat{s}^{2}\left\{\widehat{\eta}_{h}\right\}=\widehat{s}^{2}\left\{x_{h}^{\top} \widehat{\beta}\right\}=x_{h}^{\top} \widehat{s}^{2}\{\widehat{\beta}\} x_{h},
$$

onde $\widehat{s}^{2}\{\widehat{\beta}\}$ é uma estimativa da matriz de variância-covariância de $\beta$. Os limites de confiança inferior e superior de $\eta_{h}$, com coeficiente de confiança $\gamma=1-\alpha$ em grandes amostras, são dados por

$$
I=\widehat{\eta}_{h}-z_{\alpha / 2} \sqrt{\widehat{s}^{2}\left\{\widehat{\eta}_{h}\right\}}
$$

$\mathrm{e}$

$$
S=\widehat{\eta}_{h}+z_{\alpha / 2} \sqrt{\widehat{s}^{2}\left\{\widehat{\eta}_{h}\right\}}
$$

2) Aqui será feita a conversão do intervalo de confiança de $\eta_{h}$ para um intervalo de confiança para a resposta média $\mu_{h}$ e, para tal, será usada a seguinte relação da função de ligação,

$$
\mu_{h}=E\left(Y_{h}\right)=\exp \left(x_{h}^{\top} \beta\right)=\exp \left(\eta_{h}\right) .
$$

Assim, um intervalo de confiança para $\mu_{h}$ com coeficiente de confiança $\gamma=1-\alpha$ tem seus limites inferior e superior, respectivamente, dados por

$$
I^{*}=\exp (I) \text { e } \quad S^{*}=\exp (S)
$$

Vale destacar que os limites do intervalo de confiança de $\mu_{h}$ não são simétricos em torno da estimativa pontual $\widehat{\mu}_{h}$, isto porque $\mu_{h}$ não é uma função linear de $\eta_{h}$.

Se o objetivo é estimar várias respostas médias $\mu_{h}$ 's que correspondem a diferentes vetores $x_{h}$ 's com coeficiente de confiança conjunto $\gamma=1-\alpha$, o procedimento de Bonferroni deve ser usado. O procedimento para obter $g$ intervalos de confiança é o mesmo usado para obter um único intervalo de confiança (como explicado acima), a única diferença é que $z_{\alpha / 2}$ deve ser substituído por $z_{\alpha /(2 g)}$.

Como já destacado anteriormente, após ajustar um modelo, é indispensável verificar se o mesmo é adequado para representar os dados em estudo. A análise de diagnóstico do modelo binomial negativo é muito similar à apresentada na Seção 2.5. Deve-se observar, por exemplo, se os valores ajustados por esse modelo são próximos dos observados, se ocorrem observações discrepantes em relação às demais, e principalmente verificar se a suposição de distribuição binomial negativa feita com relação à variável resposta $Y$ está satisfeita. 
Os resíduos de Pearson do modelo binomial negativo, pela definição apresentada em (2.11), são dados por

$$
r_{i}=\frac{y_{i}-\widehat{\mu_{i}}}{\sqrt{\widehat{\mu}_{i}+\widehat{\mu}_{i}^{2} / k}}
$$

E o resíduo do desvio, como definido em (2.10), será

$$
\operatorname{dev}_{i}=\operatorname{sinal}\left(y_{i}-\widehat{\mu_{i}}\right) 2\left\{y_{i}\left[\log \left(\frac{y_{i}}{y_{i}+k}\right)-\log \left(\frac{\widehat{\mu_{i}}}{\widehat{\mu}_{i}+k}\right)\right]+k \log \left(\frac{\widehat{\mu_{i}}+k}{y_{i}+k}\right)\right\}^{1 / 2} .
$$

Uma medida de alavanca para o modelo de regressão log-linear binomial negativo é dada por $h_{i}=k \mu_{i} /\left(k+\mu_{i}\right) x_{i}^{\top}\left(X^{\top} W X\right)^{-1} x_{i}$. Com os resultados apresentados acima, uma aproximação da distância de Cook, dada por $r_{i}^{2} h_{i} /\left(1-h_{i}\right)^{2}$, pode ser facilmente calculada. Svetliza e Paula (2001) apresentam outras medidas de influência parà o modelo log-linear binomial negativo. Se $k$ for desconhecido, este pode ser substituído por $\widehat{k}$.

A construção do gráfico de probabilidade meio-normal com envelope simulado para os resíduos do desvio do modelo binomial negativo é feita como descrito na Seção 2.5, onde o passo (1) nesse caso é

1. ajuste o modelo de regressão binomial negativo para obter $\widehat{\mu}_{1}, \ldots, \widehat{\mu}_{n}$ e $\widehat{k}$, e gere uma amostra simulada $y_{1}^{*}, \ldots, y_{n}^{*}$ de $n$ observações independentes, onde $Y_{i}^{*} \sim \mathrm{BN}\left(\widehat{k}, \widehat{\mu}_{i}\right)$. Se $k$ for conhecido, ajuste um MLG com distribuição binomial negativa para obter $\widehat{\mu}_{1}, \ldots, \widehat{\mu}_{n}$, e gere uma amostra simulada $y_{1}^{*}, \ldots, y_{n}^{*}$ de $n$ observações independentes, onde $Y_{i}^{*} \sim \mathrm{BN}\left(k, \widehat{\mu}_{i}\right)$.

Os demais passos são iguais aos apresentados na Seção 2.5 .

Uma técnica gráfica para detectar superdispersão é discutida por Lambert e Roeder (1995), e uma verificação formal é descrita por Lawless (1987). Assuma que o modelo de regressão binomial negativo é correto e seja $\delta=1 / k$. Se $\delta=0$, não há presença de superdispersão e os dados procedem de uma distribuição de Poisson. A estatística da razão de verossimilhanças de $\mathrm{H}_{0}: \delta=0$ contra $\mathrm{H}_{1}: \delta \neq 0$ é $\omega=-2\left(l_{P}-l_{N B}\right)$, onde $l_{P}$ e $l_{N B}$ são os logaritmos das funções de verossimilhança maximizadas dos modelos de Poisson e binomial negativo, respectivamente. Observe que o valor de $\delta$ estabelecido em $\mathrm{H}_{0}$ encontra-se na borda do espaço paramétrico e, portanto, as propriedades usuais do teste da razão de verossimilhanças não são válidas. Lawless (1987) mostra que, sob $\mathrm{H}_{0}$, a função distribuição acumulada de $\omega$ é $F_{\omega}(z)=1 / 2+P\left(\chi_{1}^{2} \leq z\right)$, para $z \geq 0$, onde $\chi_{1}^{2}$ representa uma variável aleatória de distribuição qui-quadrado com um grau de liberdade.

Paul et al. (1989) e Dean (1992) apresentam outras técnicas de diagnóstico mais complexas. 
Vale destacar que outra alternativa para a análise de contagens superdispersas são os modelos de quase-verossimilhança (Wedderburn, 1974 ou David, 2000).

\subsection{Risco Relativo}

O risco relativo, que também é conhecido como razão de riscos (ambos abreviados por RR), é uma medida de grande interesse e efeito prático, principalmente em estudos epidemiológicos. No contexto dos MLG's, o risco relativo expressa o impacto de cada uma das covariadas na variável resposta, e os parâmetros $\beta$ do modelo de regressão estão associados ao risco relativo de cada variável explicativa.

A média da variável resposta e os parâmetros de regressão estão relacionados pela função de ligação $g(\cdot)$. Pode haver mais do que uma função de ligação aparentemente apropriada a uma particular aplicação. A função de ligação logarítmica $g\left(\mu_{i}\right)=\log \mu_{i}$ tem uma vantagem sobre outras possíveis funções de ligação usuais pois permite uma interpretação simples para os parâmetros de regressão.

Sejam $x_{h}=\left(1, \ldots, x_{h j}, \ldots, x_{h p}\right)^{\top}$ um vetor de valores das covariadas e $x_{h^{*}}=$ $\left(1, \ldots, x_{h j}+c, \ldots, x_{h p}\right)^{\top}$. Sob um modelo log-linear, as médias da variável resposta dados $x_{h}$ e $x_{h^{*}}$ são $\mu_{h}=\exp \left(x_{h}^{\top} \beta\right)$ e $\mu_{h^{*}}=\exp \left(x_{h^{*}}^{\top} \beta\right)$ respectivamente. O risco relativo para a $j$-ésima covariada é dado por

$$
R R_{j}=\frac{\mu_{h^{*}}}{\mu_{h}}=\frac{\exp \left(x_{h^{*}}^{\top} \beta\right)}{\exp \left(x_{h}^{\top} \beta\right)}=\exp \left(c \beta_{j}\right)
$$

e, portanto, $c \beta_{j}$ é o logaritmo desse risco quando o valor da $j$-ésima covariada é acrescido de $c$ unidades e as outras covariadas permanecem inalteradas. Note que $R R_{j}$ não depende de $x_{h j}$. Assim, uma estimativa desse risco é dada por

$$
\widehat{R R}_{j}=\frac{\widehat{\mu}_{h^{*}}}{\widehat{\mu}_{h}}=\exp \left(c \widehat{\beta}_{j}\right) \text {. }
$$

Logo, temos que $\exp \left(\widehat{\beta}_{j}\right)$ é uma estimativa do risco relativo ao aumento de uma unidade no valor da $j$-ésima covariada, mantendo as outras covariadas constantes.

Pelos resultados apresentados na Seção 2.4 podemos construir um intervalo de confiança assintótico para o risco relativo ao aumento de $c$ unidades na $j$-ésima covariada quando as outras covariadas permanecem inalteradas, que será dado por

$$
\exp \left[c\left(\widehat{\beta}_{j} \mp z_{\alpha / 2} \sqrt{\widehat{s}_{j j}^{2}\{\hat{\beta}, \widehat{k}\}}\right)\right],
$$

onde $z_{\alpha}$ é o quantil de ordem $1-\alpha$ de distribuição normal padrão e $\widehat{s}_{j j}^{2}\{\widehat{\beta}, \widehat{k}\}$ é o elemento $(j, j)$ da matriz $s^{2}\{\widehat{\beta}, \widehat{k}\}$, dada em $(2.21)$, avaliada em $(\widehat{\beta}, \widehat{k})$. Se o valor um não pertencer a esse intervalo temos evidências, com um nível de significância 
$\alpha$, de que o aumento de $c$ unidades na $j$-ésima covariada altera a resposta média, indicando que essa covariada causa impacto na variável resposta, considerando constante as demais covariadas. Vale destacar que essa interpretação é equivalente ao teste de hipótese nula $\beta_{j}$ igual a zero.

A apresentação dos resultados do ajuste de um modelo log-linear através do risco relativo é muito comum em estudos de associação entre poluição atmosférica e saúde humana. Vários trabalhos têm sido publicados em diversos países e a comparação desses resultados torna-se difícil uma vez que cada autor apresenta o risco relativo a um determinado incremento do poluente (medida $c$ dada em (2.24)). Singer et al. (2002) propõem a construção de uma curva de risco relativo com bandas de confiança, onde a estimativa do $R R$ e respectivo intervalo de confiança são calculados continuamente para diferentes incrementos $c$ do poluente, e a amplitude de variação de $c$ é determinada por valores plausíveis do ponto de vista prático. Vale destacar que essa forma de apresentação dos resultados de um modelo log-linear através do risco relativo pode ser empregada em diversas outras áreas e apresenta maior facilidade de interpretação, mesmo para pessoas sem muito conhecimento de modelagem estatística. 


\section{Capítulo 3}

\section{Modelos Aditivos Generalizados}

\subsection{Definição}

Os modelos aditivos generalizados (MAG) estendem a classe dos MLG's permitindo não linearidade na relação entre uma função da média da variável resposta e as covariáveis (Hastie e Tibshirani, 1990). Se $x_{1}, \ldots, x_{n}$ são um conjunto de $n$ covariadas de dimensão $p \operatorname{com} x_{i 1} \equiv 1$ para todo $n$ e se $\mu_{i}$ indica a média da variável resposta correspondente à $i$-ésima observação, como visto no Capítulo 2 , os MLG's impõem que

$$
\eta_{i}=g\left(\mu_{i}\right)=\beta_{1}+\sum_{j=2}^{p} \beta_{j} x_{i j}
$$

enquanto que os MAG's admitem que

$$
\eta_{i}=g\left(\mu_{i}\right)=\beta_{1}+\sum_{j=2}^{p} f_{j}\left(x_{i j}\right)
$$

onde cada $f_{j}(\cdot)$ é uma função contínua não paramétrica estimada através de curvas de alisamento, também chamadas de alisadores ou suavizadores (Hastie e Tibshirani, 1990). Um caso particular desse último é o modelo aditivo generalizado parcialmente linear, também conhecido como semi-paramétrico

$$
\eta_{i}=g\left(\mu_{i}\right)=\beta_{1}+\sum_{j=2}^{p_{1}} \beta_{j} x_{i j}+\sum_{j=p_{1}+1}^{p} f_{j}\left(x_{i j}\right),
$$

cujo preditor linear combina termos paramétricos para $p_{1}$ covariadas e termos não paramétricos para as demais $\left(p-p_{1}\right)$ covariadas. Daqui por diante denotaremos por $f_{j}$ o vetor de valores da função $f_{j}(\cdot)$ nos $n$ valores da $j$-ésima covariada, isto é, $f_{j}=\left(f_{j}\left(x_{1 j}\right), \ldots, f_{j}\left(x_{n j}\right)\right)^{\top}$. 
Na prática, o interesse pelo estudo de associações entre variáveis que não apresentam relação linear é muito comum. Quando essa relação não é linear mas apresenta uma forma conhecida (paramétrica), a utilização da função de ligação $g\left(\mu_{i}\right)$ e a aplicação dos MLG's pode ser muito útil. Caso contrário, os MAG's são uma boa alternativa como técnica de análise. A vantagem dos MAG's é que nesses modelos a relação entre as variáveis resposta e explicativas não apresenta forma paramétrica; os dados indicam qual a forma da relação funcional mais apropriada. Por outro lado, os MAG's apresentam como desvantagem um método de ajuste mais complexo e uma forma de apresentação dos resultados mais difícil de ser interpretada. Os MLG's e os MAG's podem ser aplicados em situações similares, apesar de apresentarem propósitos analíticos diferentes. Os MLG's enfatizam a estimação e análise inferencial dos parâmetros do modelo, enquanto os MAG's focalizam a exploração dos dados de forma não paramétrica, e são mais adequados para a exploração de conjuntos de dados e a visualização da relação entre as variáveis resposta e explicativas.

Todos os resultados e procedimentos apresentados no decorrer desse capítulo serão, em geral, ilustrados em conjuntos de dados simulados. No diagrama de dispersão da Figura 3.1 temos 600 observações independentes onde cada $y_{i}$ foi gerada de forma aleatória segundo uma distribuição binomial negativa com parâmetro de dispersão $k=10$ e média $\mu_{i}=e^{4}\left(2+\operatorname{sen} x_{i}\right)$, e $x_{i}$ foi gerado de forma independente segundo uma distribuição Uniforme $(0,4 \pi)$.

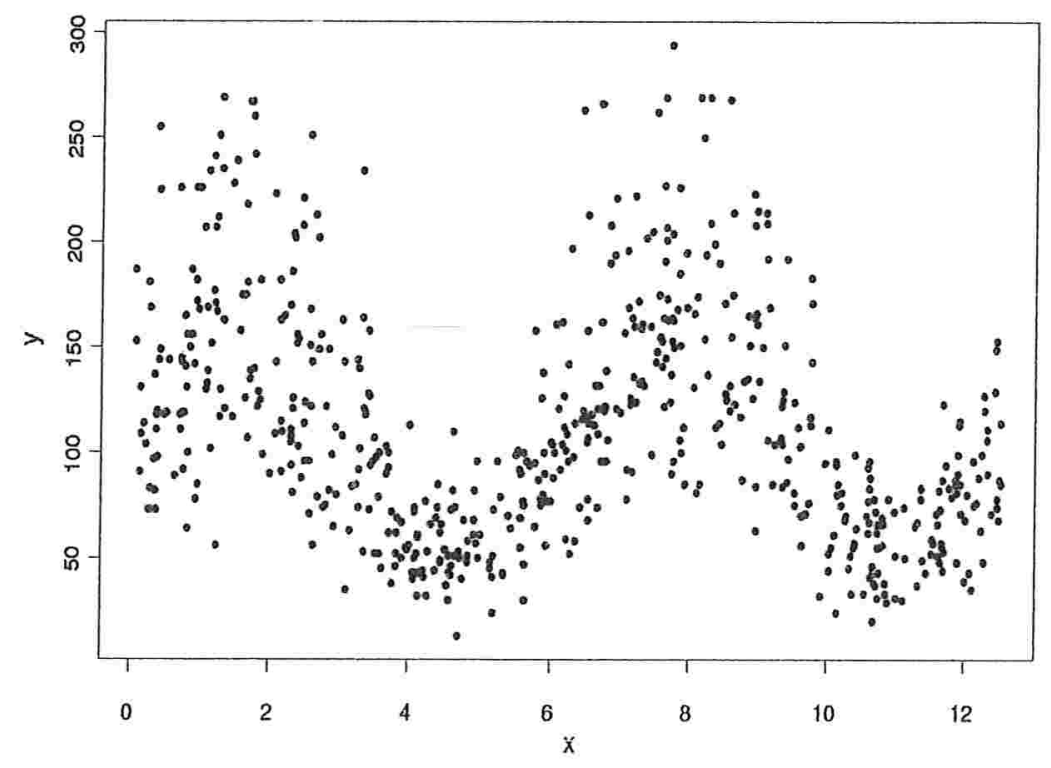

Figura 3.1: Diagrama de dispersão para dados simulados. 
Assumir que a relação entre as variáveis $x$ e $y$ apresentadas na Figura 3.1 é linear parece absurdo. A Figura 3.2 apresenta os resultados dos ajustes dos modelos de regressão com distribuições de Poisson e binomial negativa, ambos com função de ligação logarítmica (todos os detalhes sobre esses ajustes estão apresentados no Capítulo 2). Os valores ajustados pelos dois modelos são praticamente iguais (curvas de ajuste sobrepostas), e ambos os resultados não representam de forma adequada a relação real entre as variáveis $x$ e $y$. Temos aqui um caso típico onde o ajuste de um MAG é adequado para o estudo da associação entre as variáveis $x$ e $y$.

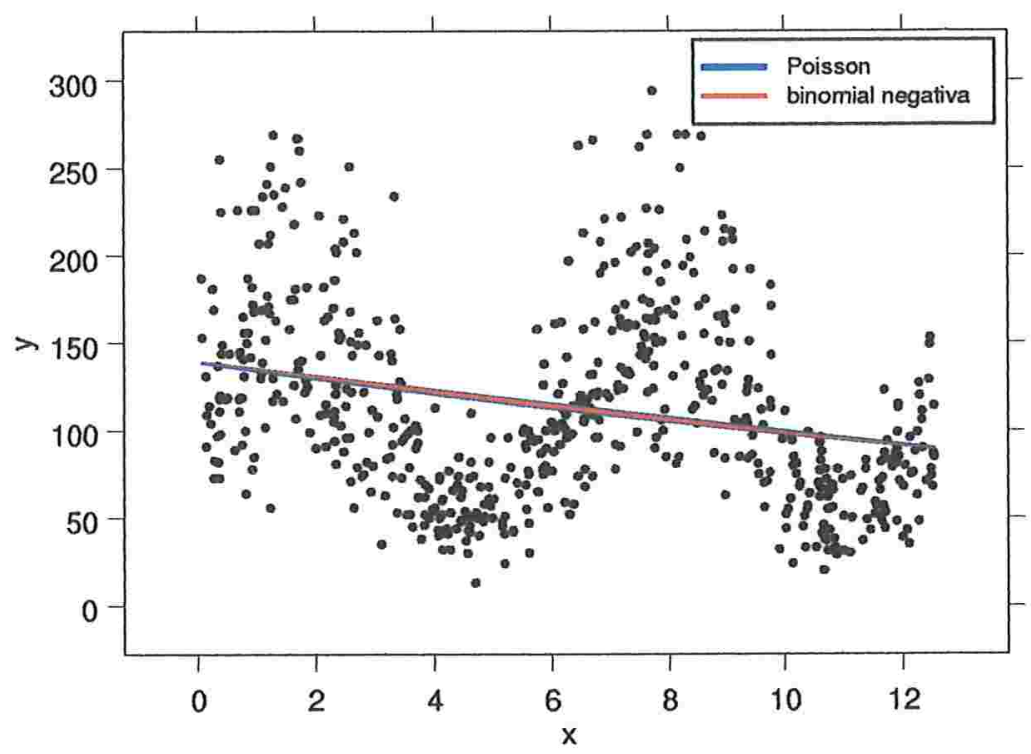

Figura 3.2: Diagrama de dispersão e ajuste dos modelos de regressão com distribuições de Poisson e binomial negativa, ambos com função de ligação logarítmica.

Tanto o ajuste dos MAG's quanto a análise inferencial sobre seus componentes foram desenvolvidos em analogia aos procedimentos utilizados com esses objetivos nos MLG's, modificando-os de forma que $f_{2}, \ldots, f_{p}$ em (3.2) sejam estimadas através dos alisadores (ou suavizadores). Por este motivo, os métodos de suavização são de extrema importância no processo de ajuste dos MAG's e serão apresentados na Seção 3.2.

O estudo geral dos MAG's do ponto de vista teórico pode ser encontrado em Hastie e Tibshirani (1990). Já do ponto de vista prático, esses modelos apresentam um custo computacional elevado (Chambers e Hastie, 1993), e os resultados apresentados na literatura são escassos. Todos esses conceitos serão apresentados 
nas Seções 3.3 a 3.5, e são válidos quando a distribuição da variável resposta pertence à família exponencial. No entanto, a distribuição binomial negativa, como mencionada no Capítulo 2, só pertence à família exponencial quando o parâmetro $k$ é conhecido, o que na prática é uma situação irreal. Recentemente, Thurston, Wand e Wiencke (2000) estenderam os MAG's para englobar respostas binomiais negativas. Eles indicam os passos para a implementação de um algoritmo iterativo para o ajuste do modelo aditivo binomial negativo e mostram como inferências devem ser realizadas com base nesse modelo. Na Seção 3.6 esses pontos serão apresentados e discutidos detalhadamente.

De forma análoga ao capítulo anterior, na seção final (Seção 3.7) destaque será dado à medida risco relativo, que também apresenta grande importância na interpretação dos resultados dos MAG's com função de ligação logarítmica.

\subsection{Alisadores}

Um alisador (ou suavizador) é uma ferramenta que descreve a tendência de uma variável $y$ como função de uma ou mais variáveis. Quando a tendência de $y$ é descrita em função de apenas uma variável $x$, o alisador é denominado unidimensional, e quando $p$ variáveis são consideradas, diz-se que o alisador é multidimensional. Aqui vamos trabalhar apenas com alisadores unidimensionais, e assumir, a princípio, que não ocorrem empates em $x$.

Formalmente, um alisador é uma função de $x=\left(x_{1}, \ldots, x_{n}\right)^{\top}$ e $y=\left(y_{1}, \ldots\right.$, $\left.y_{n}\right)^{\top}$, digamos $f\left(x_{i}\right)=f\left(x_{i} \mid y_{i}\right)$, com mesmo domínio de $x$ ou, algumas vezes, definida apenas para os $x_{i}$ da amostra (neste caso algum tipo de interpolação é necessário para obter estimativas associadas a outros valores de $x$ ). Assim, temos que para cada valor $x_{i}$ o alisador associa um valor $f\left(x_{i}\right)$ cuja estimativa $\widehat{f}\left(x_{i}\right)$ é obtida no procedimento de suavização com base em $v$ observações vizinhas. Os pontos $\left(x_{i}, \widehat{f}\left(x_{i}\right)\right)$ são apresentados graficamente resultando em uma curva suavizada ou curva de alisamento, e os valores desta função devem, por definição, ser mais "suaves" do que os valores de $y$, ou seja, devem ter menor variabilidade do que os valores de $y$. Esta é a razão do nome suavizador ou alisador.

As curvas suavizadas podem ser utilizadas simplesmente para descrever a tendência dos dados em diagramas de dispersão (Schwartz, 1994), para verificar a adequação de um modelo de regressão linear simples (Neter et al., 1996) ou para estudar a dependência da média de uma variável resposta $Y$ em função de covariadas, que é o nosso objetivo com o ajuste dos MAG's. Lima (2001) descreve as aplicações dos alisadores com maiores detalhes. 
Para ilustração dos alisadores, vamos assumir o seguinte modelo

$$
y_{i}=f\left(x_{i}\right)+\varepsilon_{i}, \quad i=1, \ldots, n,
$$

onde $f\left(x_{i}\right)$ é uma função arbitrária desconhecida e os $\varepsilon_{i}$ 's são erros não correlacionados com média zero e variância comum $\sigma^{2}$. Este modelo pode ser visto como uma generalização de um modelo de regressão linear simples (Neter et al., 1996).

Um alisador é dito linear quando o vetor de valores previstos $\widehat{y}=\widehat{f}=$ $\left(\widehat{f}\left(x_{1}\right), \ldots, \widehat{f}\left(x_{n}\right)\right)^{\top}$ pode ser escrito como $\widehat{f}=S y$, onde $S$ é uma matriz de dimensão $n \times n$ chamada matriz alisadora, que não depende de $y$. Assim, temos que

$$
\widehat{y}_{i}=\sum_{j=1}^{n} s_{i j} y_{j}
$$

onde $s_{i j}$ são os pesos da matriz alisadora linear

$$
S=\left(\begin{array}{cccccc}
s_{11} & s_{12} & \ldots & s_{1 i} & \ldots & s_{1 n} \\
s_{21} & s_{22} & \ldots & s_{2 i} & \ldots & s_{2 n} \\
\vdots & \vdots & \ddots & \vdots & & \vdots \\
s_{i 1} & s_{i 2} & \ldots & s_{i i} & \ldots & s_{i n} \\
\vdots & \vdots & & \vdots & \ddots & \vdots \\
s_{n 1} & s_{n 2} & \ldots & s_{n i} & \ldots & s_{n n}
\end{array}\right)
$$

Nesse caso a matriz $S$ desempenha papel semelhante à matriz chapéu (hat) dos modelos de regressão linear, e algumas condições impostas a $S$ (Hoaglin e Welsch, 1978) são

$$
\begin{aligned}
& \text { i. } 0 \leq s_{i i} \leq 1, \\
& \text { ii. }-1 \leq s_{i j} \leq 1, \text { para } i \neq j, \\
& \text { iii. } \sum_{j=1}^{n} s_{i j}=1,
\end{aligned}
$$

dentre outras, porém $S$ não é simétrica nem idempotente.

Uma estimativa bastante simples para $f\left(x_{i}\right)$ é a média dos valores de $y$ correspondentes a valores próximos de $x_{i}$, chamada de média móvel. Esses "valores próximos" podem ser definidos, por exemplo, a partir de um conjunto que inclui o próprio $x_{i}$ além de $m$ pontos à sua direita e $m$ pontos à sua esquerda, quando existirem $(v=2 m+1)$. A Figura 3.3 apresenta a função média móvel estimada com $m=20$, para os conjuntos de dados apresentados na Figura 3.1. Essa função 
alisadora é linear e, como ilustração, temos que a matriz alisadora para a média móvel com $m=2(v=5)$ e $n=10$ é dada por

$$
S=\left(\begin{array}{cccccccccc}
\frac{1}{3} & \frac{1}{3} & \frac{1}{3} & 0 & 0 & 0 & 0 & 0 & 0 & 0 \\
\frac{1}{4} & \frac{1}{4} & \frac{1}{4} & \frac{1}{4} & 0 & 0 & 0 & 0 & 0 & 0 \\
\frac{1}{5} & \frac{1}{5} & \frac{1}{5} & \frac{1}{5} & \frac{1}{5} & 0 & 0 & 0 & 0 & 0 \\
0 & \frac{1}{5} & \frac{1}{5} & \frac{1}{5} & \frac{1}{5} & \frac{1}{5} & 0 & 0 & 0 & 0 \\
0 & 0 & \frac{1}{5} & \frac{1}{5} & \frac{1}{5} & \frac{1}{5} & \frac{1}{5} & 0 & 0 & 0 \\
0 & 0 & 0 & \frac{1}{5} & \frac{1}{5} & \frac{1}{5} & \frac{1}{5} & \frac{1}{5} & 0 & 0 \\
0 & 0 & 0 & 0 & \frac{1}{5} & \frac{1}{5} & \frac{1}{5} & \frac{1}{5} & \frac{1}{5} & 0 \\
0 & 0 & 0 & 0 & 0 & \frac{1}{5} & \frac{1}{5} & \frac{1}{5} & \frac{1}{5} & \frac{1}{5} \\
0 & 0 & 0 & 0 & 0 & 0 & \frac{1}{4} & \frac{1}{4} & \frac{1}{4} & \frac{1}{4} \\
0 & 0 & 0 & 0 & 0 & 0 & 0 & \frac{1}{3} & \frac{1}{3} & \frac{1}{3}
\end{array}\right)
$$

Note que a vizinhança é truncada próxima aos limites da matriz, quando as $m$ observações à direita ou as $m$ observações à esquerda de um ponto não estão disponíveis.

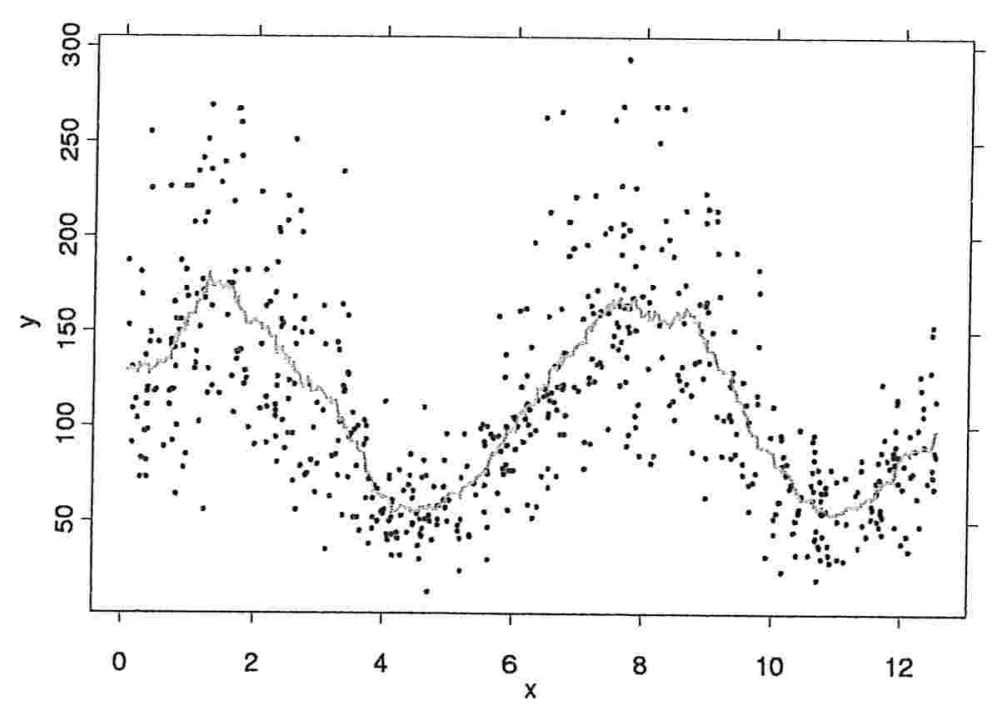

Figura 3.3: Curva de alisamento: média móvel para $m=20$.

As duas considerações mais importantes para a escolha do alisador envolvem a técnica utilizada para obtenção de $\widehat{f}$ e o tamanho da vizinhança $v$. Este último está associado ao parâmetro de alisamento (ou suavização) $\lambda$ do processo, mais conhecido como span, que corresponde à proporção do número total de observações a ser utilizado no cálculo de cada $\widehat{f}\left(x_{i}\right)$, ou seja, temos que $0<\lambda \leq 1$ e $\lambda=v / n$. O parâmetro de alisamento $\lambda$ determina o compromisso entre o viés e 
e

$$
\mathrm{gl}=\operatorname{tr}\left(2 S-S S^{\top}\right)
$$

Assim, temos que quanto maior o número de parâmetros ou graus de liberdade de um alisador, menor o grau de suavização, menor o valor de $\lambda$.

No modelo de regressão linear temos que $\sum \operatorname{Var}\left(Y_{i}\right)=p \sigma^{2}$, onde $p$ é o número de parâmetros do modelo, que é igual aos graus de liberdade do mesmo. Uma definição análoga para os modelos aditivos seria a dada em (3.4), que é mais utilizada para a escolha de parâmetros de algumas funções alisadoras, como por exemplo a cubic spline (para mais detalhes sobre esse alisador ver Buja et al., 1989, Seção 2, ou Marsh e Cormier, 2001). Outra motivação é que na regressão linear a matriz chapéu $H$ apresenta as seguintes propriedades

$$
\operatorname{tr}\left(H H^{\top}\right)=\operatorname{tr}(H)=\operatorname{posto}(H)=p,
$$

e como já comentado anteriormente, a matriz $S$ desempenha papel semelhante à matriz chapéu dos modelos lineares, e daí decorrem as definições (3.4) e (3.5). A motivação para a definição (3.6) é que no modelo (3.3) a soma de quadrados dos resíduos, isto é $S Q R=(y-\widehat{y})^{\top}(y-\widehat{y})$, tem esperança

$$
\mathrm{E}(S Q R)=\left[n-\operatorname{tr}\left(2 S-S S^{\top}\right)\right] \sigma^{2}+f^{\top}(I-S)^{\top}(I-S) f,
$$

onde o último termo mede viés. Como no modelo de regressão linear o termo $\operatorname{tr}\left(2 S-S S^{\top}\right)$ é igual a $p$, se estamos alisando um ruído ( $f$ é um vetor de zeros) esses valores são correspondentes. Buja et al. (1989, Seção 2.7.3) mostram que essa definição é útil para a comparação de dois alisadores e apresenta um estudo mais detalhado dos graus de liberdade de um alisador linear.

A utilização de um alisador requer a fixação de um parâmetro de suavização ou alisamento $\lambda$. A escolha do parâmetro $\lambda$ é um problema de grande importância, e pode ser resolvido, por exemplo, após o teste de vários valores de $\lambda$ para um mesmo conjunto de dados avaliados graficamente, ou então pelo procedimento conhecido como validação cruzada (cross-validation). A validação cruzada é um método automático de alto custo computacional, pois para cada $\lambda$ esse método consiste em retirar o ponto $\left(x_{i}, y_{i}\right)$ e calcular $\widehat{f}\left(x_{i}\right)$ com base nos $n-1$ pontos restantes, para $i=1, \ldots, n$. A função validação cruzada para o modelo (3.3) é definida como

$$
\mathrm{VC}(\lambda)=\frac{1}{n} \sum_{i=1}^{n}\left(y_{i}-\widehat{f}_{\lambda}^{-(i)}\left(x_{i}\right)\right)^{2}
$$

onde $\widehat{f}_{\lambda}^{-(i)}\left(x_{i}\right)$ é a estimativa de $f\left(x_{i}\right)$ obtida com parâmetro de suavização $\lambda$ quando o ponto $\left(x_{i}, y_{i}\right)$ é omitido (Hastie e Tibshirani, 1990, Seção 3.4̣.3). A 
função (3.7) é calculada para um conjunto pré-fixado de $\lambda$ 's, sendo selecionado o valor de $\lambda$ que a minimiza. Uma outra forma de escolher o parâmetro de suavização é avaliar através de técnicas de diagnóstico, para qual valor de $\lambda$ o modelo proposto apresenta melhor qualidade de ajuste.

Existem diversas técnicas de suavização propostas na literatura e o que difere uma das outras é a forma de cálculo das estimativas $\widehat{f}\left(x_{i}\right)$. Hastie e Tibshirani (1990), Buja et al. (1989) e Lima (2001) descrevem uma série de alisadores e apresentam bibliografia adicional sobre o assunto. A Figura 3.4 ilustra algumas curvas suavizadas por diferentes técnicas com o auxílio do software S-Plus. A escolha de um alisador é subjetiva, e pode ser baseada em considerações teóricas ou mesmo no grau de facilidade para sua obtenção. Aqui vamos descrever com maiores detalhes apenas o método de alisamento locally weighted regression smoother, conhecido como loess, que será utilizado posteriormente no ajuste dos MAG's e do modelo binomial negativo. No entanto, os demais alisadores propostos na literatura podem da mesma forma ser utilizados no ajuste desses modelos.

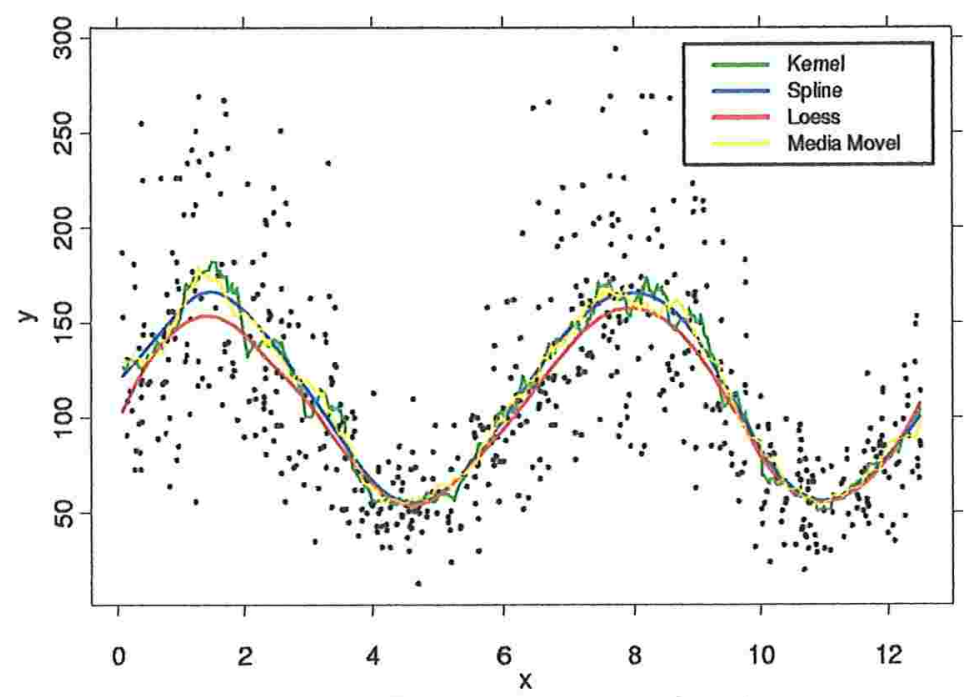

Figura 3.4: Diferentes curvas de alisamento.

Apesar de assumirmos que os dados não apresentam réplicas em $x$, na prática esse fato é muito comum. Nesse caso, Lima (2001, Seção 2.6) apresenta uma solução simples que pode ser usada para todos os alisadores. Essa solução consiste na formação de um novo conjunto de dados com $n^{\prime}<n$ observações determinadas pelos $n^{\prime}$ valores distintos de $x$, onde deve ser aplicado um procedimento de suavização ponderada.

O loess, proposto por Cleveland (1979), é um alisador baseado no ajuste sucessivo de modelos de regressão pelo método dos mínimos quadrados ponderados. 
Cada modelo de regressão é ajustado considerando observações com valores de $x$ pertencentes a uma vizinhança da coordenada $x_{i}$ de uma observação $\left(x_{i}, y_{i}\right)$ fixada e denominada ponto alvo, $i=1, \ldots, n$. O valor ajustado é $\widehat{y}_{i}=\widehat{f}\left(x_{i}\right)$. Portanto, considerando sucessivamente as $n$ observações $\left(x_{i}, y_{i}\right)$ como ponto alvo, obtêm-se os pontos $\left(x_{i}, \widehat{f}\left(x_{i}\right)\right), i=1, \ldots, n$, que geram a curva suavizada.

Para cada ponto alvo $\left(x_{i}, y_{i}\right)$ define-se uma vizinhança formada por $v$ pares de observações $\left(x_{j}, y_{j}\right)$ que possuem as coordenadas $x_{j}$ mais próximas de $x_{i}$. Assim, o parâmetro de alisamento da função loess é dado por $\lambda=v / n$. Em seguida é ajustado um polinômio de grau $d, y_{j}=\alpha+\beta_{1} x_{j}+\ldots+\beta_{d} x_{j}^{d}+e_{j}$, com $\mathrm{E}\left(e_{j}\right)=0$ e $\operatorname{Var}\left(e_{j}\right)=\sigma^{2}, j=1, \ldots, n$, pelo método dos mínimos quadrados ponderados, sendo $U_{x_{i}}$ a matriz de pesos associada a cada ajuste local.

O grau do polinômio $d$ deve ser fixado com base no padrão apresentado pelos dados num diagrama de dispersão. De uma forma geral, se a nuvem de pontos sugere uma tendência sem máximos ou mínimos locais, então um ajuste linear $(d=1)$, é adequado. Mas se existirem regiões com máximos ou mínimos locais, o ajuste quadrático $(d=2)$ normalmente produz uma curva que melhora a descrição do padrão dos dados localmente.

A matriz de pesos associada ao ponto alvo $\left(x_{i}, y_{i}\right)$ em cada ajuste local é dada por

$$
U_{x_{i}}=\operatorname{diag}\left(K\left(\frac{x_{1}-x_{i}}{h_{i}}\right), \ldots, K\left(\frac{x_{n}-x_{i}}{h_{i}}\right)\right),
$$

onde $h_{i}$ é a distância entre $x_{i}$ e o seu $v$-ésimo vizinho mais próximo e $K(\cdot)$ é uma função simétrica positiva conhecida como função kernel tricúbica, que é dada por

$$
K(z)= \begin{cases}\left(1-|z|^{3}\right)^{3} & \text { para }|z|<1 \\ 0 & \text { para }|z| \geq 1\end{cases}
$$

Assim, temos que o peso do ponto alvo $\left(x_{i}, y_{i}\right)$ em seu ajuste local é 1 , os pesos dos pontos que formam sua vizinhança diminuem à medida que se afastam de $x_{i}$, e pesos nulos são dados aos pontos fora de sua vizinhança. Lima (2001, Seção 2.2) e Fox (2000, Seção 3) apresentam um estudo mais detalhado dessa função.

Como o loess é um alisador linear, assumindo o modelo (3.3) com uma função alisadora loess de parâmetros $\lambda$ e $d$, sua matriz alisadora $S$ depende somente de $x$ e cada linha $s_{i}$ de $S, i=1, \ldots, n$, é dada pela $i$-ésima linha da matriz

$$
S_{x_{i}}=\widetilde{X}\left(\tilde{X}^{\top} U_{x_{i}} \widetilde{X}\right)^{-1} \tilde{X}^{\top} U_{x_{i}}
$$

onde $\widetilde{X}$ é uma matriz com linhas $\left(1, x_{i}, x_{i}^{2}, \ldots, x_{i}^{d}\right)$.

As Figuras 3.5a e 3.5b ilustram a obtenção da função $\widehat{f}$ pelo método de alisamento loess com parâmetros $d=1$ e $\lambda=30 \%$, e $d=2$ e $\lambda=50 \%$, respectivamente. Nessas figuras, a linha vertical laranja indica a ordenada do ponto 
alvo $\left(x_{i}, y_{i}\right)$, os círculos azuis representam os pesos relacionados a cada ponto da vizinhança calculados pela função kernel tricúbica. A vizinhança está delimitada pelas linhas tracejadas verticais vermelhas, com base nos respectivos parâmetros de alisamento $\lambda$. O modelo ajustado para o ponto alvo $\left(x_{i}, y_{i}\right)$ pelo método dos mínimos quadrados ponderados está representado pela linha (Figuras 3.5.a) e curva (Figuras 3.5.b) vermelha, e as curvas pretas representam a curva de alisamento loess gerada após a obtenção de $\widehat{f}\left(x_{i}\right)$ para $i=1, \ldots, n$. Lima (2001, Seção 2.2) apresenta os cálculos para o ajuste de uma curva loess passo a passo.

(a) $d=1$ e $\lambda=50 \%$

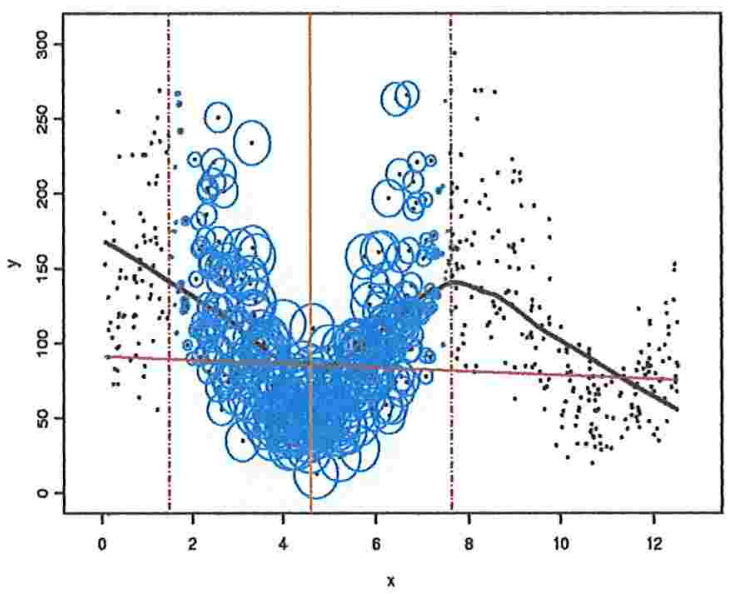

(b) $d=2$ e $\lambda=30 \%$

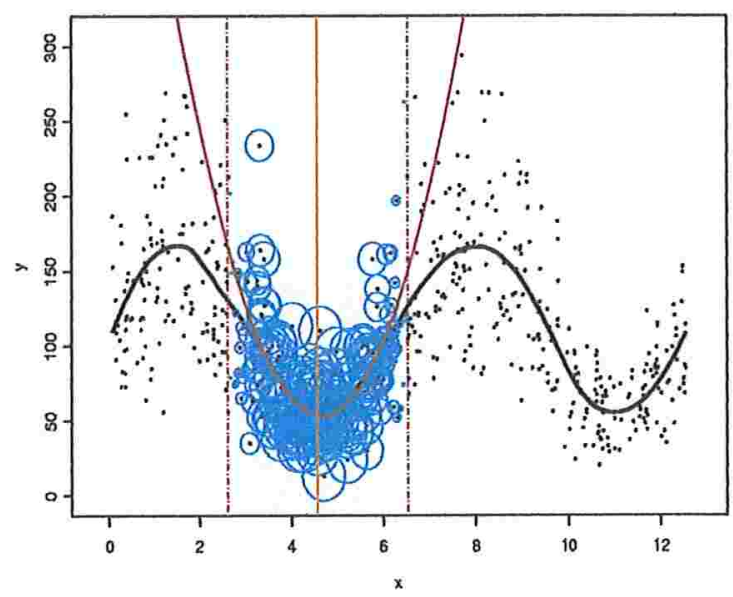

Figura 3.5: Figuras para demonstração do método de alisamento loess.

O efeito da escolha dos parâmetros $\lambda$ e $d$ está ilustrado na Figura 3.6. Como já comentado anteriormente, essa figura ilustra que o parâmetro de alisamento $\lambda$ tem influência fundamental na variância e no viés da curva estimada, e pela natureza dos dados que o ajuste do polinômio de segundo grau parece mais adequado. Comparando visualmente os gráficos da Figura 3.6, podemos dizer que o melhor ajuste foi obtido com o polinômio de segundo grau $(d=2)$ e $\lambda=30 \%$, pois nessa curva não há superajustamento, oscilações aparentemente insignificantes, e nem a perda de padrões locais. O comando loess do software S-Plus realiza o ajuste dessas curvas.

O loess apresenta também uma versão robusta que pode ser utilizada para diminuir o peso de observações discrepantes no ajuste da curva de alisamento. Cleveland (1979) e Lima (2001, Seção 2.2) apresentam maiores detalhes desse ajuste. Hastie e Tibshirani (1990), Lima (2001) e Fox (2000) apresentam um estudo mais detalhado do alisador loess. 
$\lambda=10 \%$ e $d=1$

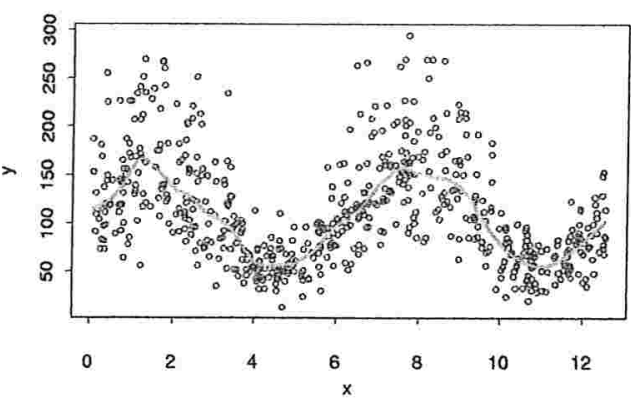

$\lambda=30 \%$ e $d=1$

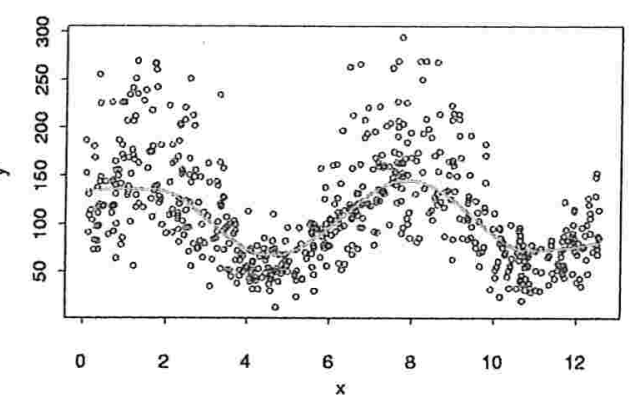

$\lambda=50 \%$ e d $=1$

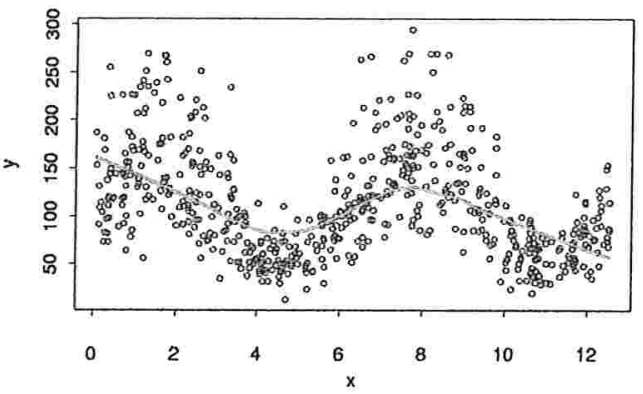

$\lambda=70 \%$ e $d=1$

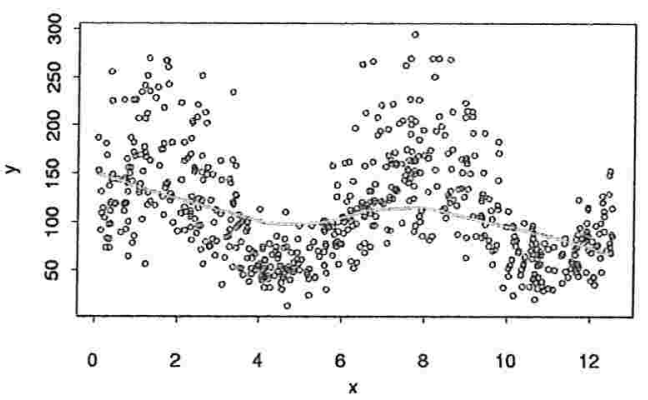

$\lambda=10 \%$ e $d=2$

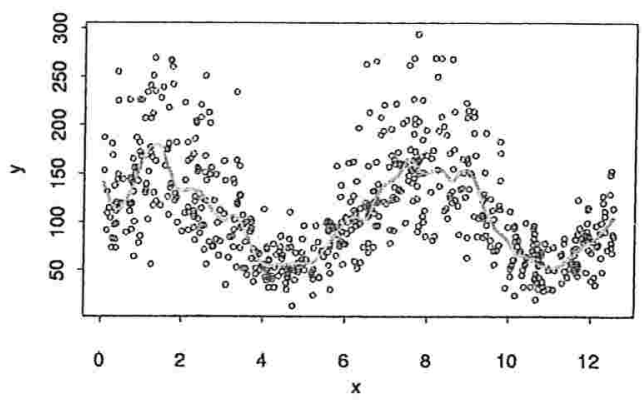

$\lambda=30 \%$ e $d=2$

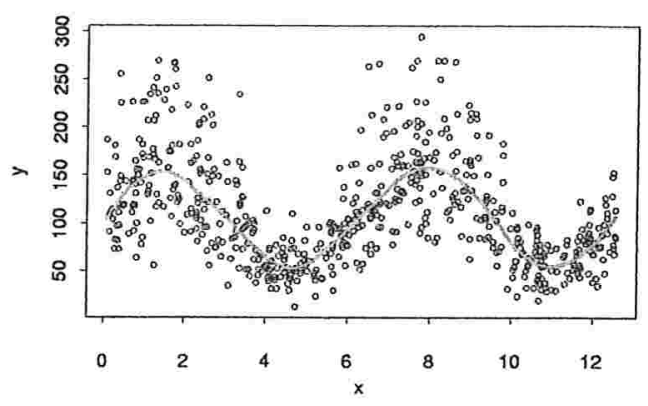

$\lambda=50 \%$ e $d=2$

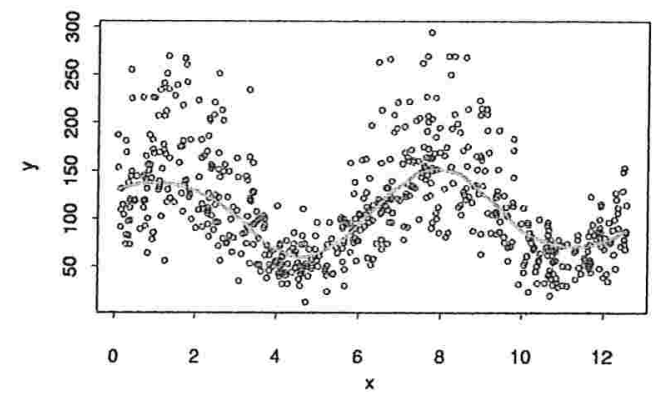

$\lambda=70 \%$ e $d=2$

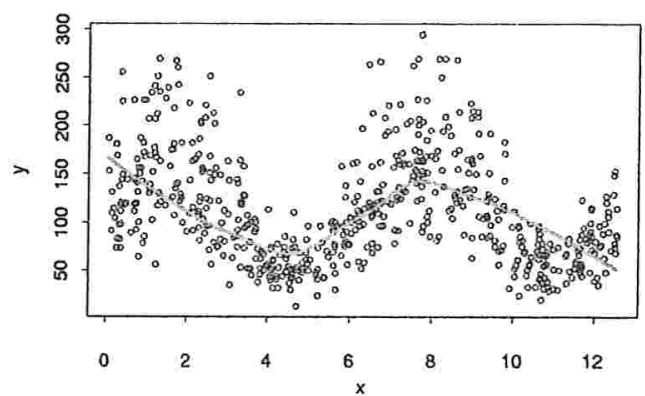

Figura 3.6: Curvas alisadas pelo método loess com diferentes valores de $\lambda$ e $d$. 


\subsection{Estimação}

A base para a estimação dos MAG's é o algoritmo de retroajuste (backfitting) utilizado também no ajuste de modelos aditivos. Assim, vamos apresentar primeiramente o procedimento de estimação dos modelos aditivos com o objetivo de facilitar a compreensão do processo de estimação dos MAG's, que será apresentado em seguida.

\subsubsection{Modelo Aditivo}

Vamos considerar primeiramente o seguinte modelo de regressão aditivo com $p$ covariadas

$$
y_{i}=\sum_{j=1}^{p} f_{j}\left(x_{i j}\right)+\varepsilon_{i}
$$

onde as $f_{j}(\cdot)$ 's são funções arbitrárias desconhecidas e os $\varepsilon_{i}$ 's são erros não correlacionados com média zero e variância comum $\sigma^{2}$. Assim, temos que

$$
\mathrm{E}\left(Y_{i} \mid x_{i}\right)=\mu_{i}=\sum_{j=1}^{p} f_{j}\left(x_{i j}\right)
$$

Para tornar única cada uma das funções $f_{j}=\left(f_{j}\left(x_{1 j}\right), \ldots, f_{j}\left(x_{n j}\right)\right)^{\top}$, o modelo (3.9) assume que $\sum_{i=1}^{n} f_{j}\left(x_{i j}\right)=0$, para $j=1, \ldots, p$.

O cálculo de esperanças condicionais estabelece uma motivação simples e intuitiva para o algoritmo de estimação desse modelo que, como já citado acima, é conhecido como algoritmo de retroajuste (backfitting). Suponha que o modelo (3.9) é de fato correto, e assuma que conhecemos $f_{1}, \ldots, f_{j-1}, f_{j+1}, \ldots, f_{p}$. Se definirmos os resíduos parciais

$$
r_{i j}=y_{i}-\sum_{k \neq j} f_{k}\left(x_{i k}\right)
$$

para $i=1, \ldots, n$, temos então que

$$
\mathrm{E}\left(Y-\sum_{k \neq j} f_{k} \mid X\right)=f_{j}
$$

que minimiza $\mathrm{E}\left(Y-\sum_{k=1}^{p} f_{k}\right)^{2}$, onde $Y$ é o vetor resposta $\left(Y_{1}, \ldots, Y_{n}\right)^{\top}$ e $X$ a matriz de covariadas. Evidentemente não conhecemos $f_{1}, \ldots, f_{j-1}, f_{j+1}, \ldots, f_{p}$, mas essa idéia estabelece um caminho para estimar cada $\widehat{f}_{j}$ dadas as estimativas $\left\{\widehat{f}_{k}, k \neq j\right\}$. Assim, precisamos substituir as esperanças condicionais em (3.10) 
por alisadores que forneçam as estimativas $\widehat{f}_{1}, \ldots, \widehat{f}_{p}$ de $f_{1}, \ldots, f_{p}$. Na prática, precisamos resolver o seguinte sistema de $n p$ equações lineares

$$
\left(\begin{array}{cccc}
I & S_{1} & \ldots & S_{1} \\
S_{2} & I & \ldots & S_{2} \\
\vdots & \vdots & \ddots & \vdots \\
S_{p} & S_{p} & \ldots & I
\end{array}\right)\left(\begin{array}{c}
\widehat{f}_{1} \\
\hat{f}_{2} \\
\vdots \\
\hat{f}_{p}
\end{array}\right)=\left(\begin{array}{c}
S_{1} \\
S_{2} \\
\vdots \\
S_{p}
\end{array}\right) y
$$

onde $y=\left(y_{1}, \ldots, y_{n}\right)^{\top}, I$ é a matriz identidade de dimensão $n \times n$, e $S_{j}$ é a matriz alisadora centralizada $n \times n$ referente à $j$-ésima covariada, para $j=1, \ldots, p$. As matrizes alisadoras lineares descritas na Seção 2.2, quando aplicadas em modelos aditivos, devem ser centralizadas de modo que satisfaçam a suposição desses modelos de que $\sum_{i=1}^{n} f_{j}\left(x_{i j}\right)=0$, para $j=1, \ldots, p$, ou seja, as matrizes $S_{j}$ devem ser substituídas por $\left(I-11^{\top} / n\right) S_{j}$, onde aqui $I$ é matriz identidade $n \times n$ e 1 é o vetor unitário $n \times 1$. Essa operação também facilita a realização de cálculos matriciais, evitando a singularidade em muitos resultados. Assim, temos que a soma de cada coluna da matriz alisadora centralizada é igual a zero, e lembrando que a soma de cada linha das matrizes alisadoras lineares é igual a 1 (propriedade ( iii) apresentada na Seção 2.2), logo, temos que a soma de cada linha das matrizes alisadoras centralizadas é também igual a zero. Vale destacar que as matrizes alisadoras podem corresponder a diferentes métodos de suavização, e no caso do loess essa matriz está apresentada na Seção 2.2 .

De (3.11) temos que

$$
\widehat{f_{j}}+S_{j}\left(\sum_{k \neq j} \widehat{f_{k}}\right)=S_{j} y
$$

e logo,

$$
\widehat{f_{j}}=S_{j}\left(y-\sum_{k \neq j} \widehat{f_{k}}\right), \quad \text { para } j=1, \ldots, p .
$$

Temos então que os valores de $f_{j}$ devem ser obtidos por meio de um procedimento de suavização dos resíduos parciais, ou seja, quando $f_{j}$ é estimado o efeito das demais covariadas é removido de $y$. Assim, podemos esquematizar o algoritmo iterativo de retroajuste (backfitting) para calcular as estimativas das funções $f_{j}$ da seguinte forma

(i) Inicialize $f_{j}=0$, para $j=1, \ldots, p$.

(ii) Calcule seqiiencialmente para $j=1, \ldots, p$

$$
f_{j}=S_{j}\left(y-\sum_{k \neq j} f_{k}\right)
$$


(iii) Repita o passo (ii) até que todas as funções individuais convirjam, ou seja, até que $\left\|f_{j}^{(m)}-f_{j}^{(m-1)}\right\| \leq \epsilon$ para todo $j$ e um valor $\epsilon$ pré-estabelecido, com $\left\|f_{j}\right\|=\left(\sum_{i=1}^{n} f_{j}^{2}\left(x_{i j}\right)\right)^{1 / 2}$ representando a norma do vetor $f_{j}$.

Vale lembrar que no caso do modelo aditivo com apenas uma função não especificada, dado em (3.3), o algoritmo de retroajuste não é necessário, pois $f$ é obtida diretamente utilizando-se um alisador, como descrito na Seção 2.

O algoritmo de retroajuste corresponde ao método de Gauss-Seidel para resolver o sistema de equações (3.11). No entanto, a partir de (3.11), estimativas para $f_{1}, \ldots, f_{p}$ podem também ser obtidas diretamente pela relação

$$
\left(\begin{array}{c}
\widehat{f_{1}} \\
\widehat{f}_{2} \\
\vdots \\
\hat{f}_{p}
\end{array}\right)=\left(\begin{array}{cccc}
I & S_{1} & \ldots & S_{1} \\
S_{2} & I & \ldots & S_{2} \\
\vdots & \vdots & \ddots & \vdots \\
S_{p} & S_{p} & \ldots & I
\end{array}\right)^{-1}\left(\begin{array}{c}
S_{1} \\
S_{2} \\
\vdots \\
S_{p}
\end{array}\right) y=P^{-1} Q y
$$

se a inversa de $P$ existe, onde $P$ e $Q$ representam as matrizes de dimensão $n p \times n p$ e $n p \times n$, respectivamente, compostas de matrizes alisadoras lineares centralizadas. Pelo menos conceitualmente essa seria uma solução direta para (3.11), mas na prática não é utilizada como uma alternativa ao algoritmo de retroajuste, uma vez que este algoritmo é mais eficiente do ponto de vista computacional. Essa solução direta apenas facilita a obtenção de expressões para as funções $\widehat{f}_{j}$ que tornam mais simples o estudo de suas propriedades e cálculos de seus erros-padrão. Logo, a partir de (3.12) temos que a matriz que produz $\widehat{f}_{j}$ a partir de $y$ pode ser definida como

$$
R_{j}=E_{j} P^{-1} Q, \text { para } j=1, \ldots, p
$$

onde $E_{j}$ é uma matriz de dimensão $n \times n p$ composta por $p$ blocos de dimensão $n \times n$, sendo o $j$-ésimo bloco a matriz identidade, e os demais elementos iguais a zero, de tal maneira que

$$
\widehat{f_{j}}=R_{j} y, \text { para } j=1, \ldots, p .
$$

Seja $f=f_{1}+\ldots+f_{p}$, então temos que

$$
\widehat{f}=R_{1} y+\ldots+R_{p} y=R y,
$$

onde $R=R_{1}+\ldots+R_{p}$ é a matriz alisadora que produz $\widehat{f}$ a partir de $y$. Note que, para funções alisadoras lineares, $R$ não depende de $y$. Mesmo assim a obtenção de $R_{j}$, e logo, de $R$ não é uma tarefa computacional simples. Hastie e Tibshirani (1990) fornecem expressões mais simples de $R$ somente para modelos com duas 
funções alisadoras. Para modelos envolvendo mais de duas funções alisadoras esses autores apresentam como sugestão para a obtenção das matrizes $R_{j}$ o ajuste de $n$ modelos aditivos com o mesmo preditor dado em (3.9), considerando como variável resposta cada uma das colunas $e_{i}$ da matriz identidade $I$ de dimensão $n \times n$. Seja $R_{i k}^{j}$ o elemento da $i$-ésima linha e $k$-ésima coluna da matriz $R_{j}$. Assim, em cada ajuste com variável resposta $e_{i}$ obtemos $\widehat{f}_{j}^{i}$ que corresponde à $i$-ésima coluna de $R_{j}$, pois $R_{j} e_{i}=\left(R_{i 1}^{j}, \ldots, R_{i n}^{j}\right)^{\top}=\widehat{f}_{j}$, para $j=1, \ldots, p$. Repetindo esse procedimento para $i=1, \ldots, n$ temos as matrizes $R_{j}$, e logo, $R=R_{1}+\ldots+R_{p}$.

Se considerarmos o modelo de regressão aditivo com intercepto, ou seja, com $p$ covariadas sendo $x_{i 1} \equiv 1$ para todo $i=1, \ldots, n$, temos

$$
y_{i}=\beta_{1}+\sum_{j=2}^{p} f_{j}\left(x_{i j}\right)+\varepsilon_{i} .
$$

Nesse caso o parâmetro desconhecido $\beta_{1}$ é estimado no primeiro passo do algoritmo de retroajuste por $\bar{y}$, e logo, o resíduo parcial para estimação das $f_{j}$ 's será

$$
S_{j}\left(y-1 \bar{y}-\sum_{k \neq j} f_{k}\right),
$$

onde aqui 1 é o vetor unitário de dimensão $n \times 1$. Mas como as matrizes alisadoras são centralizadas, temos que

$$
S_{j} 1 \bar{y}=S_{j} \frac{1}{n} 11^{\top} y=\frac{1}{n} S_{j} 11^{\top} y=0,
$$

onde aqui 0 representa um vetor $n \times 1$ de zeros. Logo, temos que

$$
S_{j}\left(y-1 \bar{y}-\sum_{k \neq j} f_{k}\right)=S_{j}\left(y-\sum_{k \neq j} f_{k}\right) .
$$

Assim, concluímos que a presença do intercepto não altera as estimativas das funções $f_{j}$, e todos os resultados apresentados para o modelo sem intercepto (3.13) podem ser aplicados ao modelo (3.9). Aqui temos também que

$$
\widehat{f}=R_{1} y+\ldots+R_{p} y=R y,
$$

onde $R_{1}=11^{\top} / n$ e os demais $R_{j}, j=2, \ldots, p$, podem ser obtidos como descrito anteriormente, mas aqui a partir de um sistema de $n(p-1)$ equações.

No caso do ajuste de um modelo de regressão aditivo ponderado com peso $W$, por exemplo, esse peso deverá ser considerado na ponderação dada pela função alisadora, ou seja, deverá ser incorporado à matriz alisadora. 


\subsubsection{Modelo Aditivo Generalizado}

O processo de estimação dos MAG's envolve a combinação de dois procedimentos iterativos:

- Procedimento de ponderação local (local scoring): similar ao procedimento iterativo de mínimos quadrados reponderados utilizado no ajuste dos MLG's (apresentado na Seção 2.2), considerando o preditor aditivo (3.2) no lugar do preditor linear (3.1);

- Retroajuste (backfitting): algoritmo iterativo responsável pela estimação seqüencial de cada função alisadora $f_{j}$, apresentado na Seção 3.3.1. No caso dos MAG's esse ajuste é realizado de forma ponderada para uma variável dependente modificada.

Alguns autores descrevem o algoritmo de ponderação local como um ciclo externo e o algoritmo de retroajuste como um ciclo interno na estimação dos MAG's, uma vez que cada iteração do primeiro algoritmo requer a implemetação do segundo.

Primeiramente vamos considerar o modelo aditivo generalizado

$$
\eta_{i}=g\left(\mu_{i}\right)=\beta_{1}+\sum_{j=2}^{p} f_{j}\left(x_{i j}\right)
$$

já apresentado no início desse capítulo. Vale lembrar que os resultados aqui apresentados são válidos quando a distribuição da variável resposta pertence à família exponencial. Assim como nos modelos aditivos, assumimos que $\sum_{i=1}^{n} f_{j}\left(x_{i j}\right)=0$ para, tornar o modelo identificável, facilitando também a realização de cálculos matriciais.

Assim como nos MLG's o processo de estimação de $\beta$ pode ser visto como uma regressão pelo método dos mínimos quadrados reponderados de uma variável dependente modificada $z$ em $X$, com pesos $W$, o algoritmo de retroajuste no ajuste dos MAG's deve ser aplicado à mesma variável dependente $z$ com pesos $W$, substituindo (3.1) por (3.2). O procedimento de estimação do modelo aditivo generalizado (3.3.2) pode ser esquematizado da seguinte forma

1. Inicialize o processo com

$$
\beta_{1}^{(0)}=g(\bar{y}) \quad \text { e } \quad f_{2}^{(0)}=\ldots=f_{p}^{(0)}=0, \text { para } m=0 .
$$

2. Considere $m=m+1$ e calcule a variável dependente modificada $z^{(m)}$ e a matriz de pesos $W^{(m)}$ da seguinte forma

$$
z_{i}^{(m)}=\eta_{i}^{(m-1)}+\left(y_{i}-\mu_{i}^{(m-1)}\right)\left(\frac{\partial \eta_{i}}{\partial \mu_{i}}\right)_{(m-1)},
$$


onde

$$
\begin{gathered}
\eta_{i}^{(m-1)}=\beta_{1}^{(m-1)}+\sum_{j=2}^{p} f_{j}^{(m-1)}\left(x_{i j}\right), \\
\mu_{i}^{(m-1)}=g^{-1}\left(\eta_{i}^{(m-1)}\right),
\end{gathered}
$$

e

$$
W^{(m)}=\operatorname{diag}\left(w_{1}^{(m)}, \ldots, w_{n}^{(m)}\right)
$$

com

$$
w_{i}^{(m)}=\left(\frac{\partial \mu_{i}}{\partial \eta_{i}}\right)_{(m-1)}^{2} \frac{1}{V\left(\mu_{i}^{(m-1)}\right)},
$$

onde $V(\cdot)$ é a função de variância apresentada na Seção 2.1 .

3. Para obter $f_{2}^{(m)}, \ldots, f_{p}^{(m)}$, ajuste um modelo aditivo ponderado com variável dependente $z^{(m)}$ e peso $W^{(m)}$ através do algoritmo de retroajuste. Ou seja, considere $\beta_{1}^{(m)}=\bar{z}^{(m)}$ e calcule seqüencialmente para $j=2, \ldots, p, 2, \ldots, p, \ldots$

$$
f_{j}=S_{j}^{(m)}\left(z^{(m)}-\beta_{1}^{(m)}-\sum_{k \neq j} f_{k}\left(x_{k}\right)\right),
$$

até que cada função individual convirja obtendo assim $f_{2}^{(m)}, \ldots, f_{p}^{(m)}$, onde $S_{j}^{(m)}, j=2, \ldots, p$, são as matrizes alisadoras ponderadas por $W^{(m)}$.

4. Para um valor de $\epsilon$ pré-estabelecido, repita os passos 2 e 3 até que

$$
\frac{\sum_{j=2}^{p}\left\|f_{j}^{(m)}-f_{j}^{(m-1)}\right\|}{\sum_{j=2}^{p}\left\|f_{j}^{(m-1)}\right\|} \leq \epsilon,
$$

ou

$$
\sum_{j=1}^{n}\left|D_{p}\left(y_{i}, \mu_{i}^{(m)}\right)-D_{p}\left(y_{i}, \mu_{i}^{(m-1)}\right)\right| \leq \epsilon
$$

lembrando que $\left\|f_{j}\right\|=\left(\sum_{i=1}^{n} f_{j}^{2}\left(x_{i j}\right)\right)^{1 / 2}$ é a norma do vetor $f_{j}$, e $D_{p}$ é a função desvio definida na Seção 2.3 .

Basicamente, temos o algoritmo de ponderação local no passo 2 descrito acima, e o algoritmo de retroajuste no passo 3. Hastie e Tibshirani (1986, Seção 5.2) mostram que esse procedimento para estimação dos MAG's também pode ser visto como um método empírico para maximizar o valor esperado do logaritmo da função de verossimilhança de $y$ dadas as $p$ covariadas em $\eta$, ou seja, determinar a aproximação aditiva $\widehat{\eta}$ tal que $\mathrm{E}(\mathrm{d} l / \mathrm{d} \eta \mid X)_{\hat{\eta}}=0$. Vale destacar que no caso dos MAG's com apenas uma função não especificada, o algoritmo de retroajuste não 
é necessário pois $f$ é obtida diretamente, utilizando-se um alisador ponderado aplicado diretamente aos resíduos $r_{i}=z_{i}-\bar{z}$ em função de $x_{i}, i=1, \ldots, n$, com matriz de pesos $W$.

Assim como no modelo aditivo, podemos dizer que cada passo do algoritmo de ajuste do MAG pode ser visto como a solução de Gauss-Seidel para o seguinte sistema de $n(p-1)$ equações lineares

$$
\left(\begin{array}{cccc}
I & S_{2}^{(m)} & \ldots & S_{2}^{(m)} \\
S_{3}^{(m)} & I & \ldots & S_{3}^{(m)} \\
\vdots & \vdots & \ddots & \vdots \\
S_{p}^{(m)} & S_{p}^{(m)} & \ldots & I
\end{array}\right)\left(\begin{array}{c}
\widehat{f}_{2}^{(m)} \\
\hat{f}_{3}^{(m)} \\
\vdots \\
\hat{f}_{p}^{(m)}
\end{array}\right)=\left(\begin{array}{c}
S_{2}^{(m)} \\
S_{3}^{(m)} \\
\vdots \\
S_{p}^{(m)}
\end{array}\right) z^{(m)}
$$

onde $S_{j}^{(m)}$ é a j-ésima matriz alisadora centralizada ponderada por $W^{(m)}$. No caso do alisador loess ser utilizado, essa matriz alisadora centralizada é dada por $\left(I-11^{\top} / n\right) \widetilde{S}_{j}^{(m)}$, onde $\widetilde{S}_{j}^{(m)}$ é a matriz cuja $i$-ésima linha corresponde à $i$-ésima linha da matriz alisadora ponderada

$$
S_{x_{j}}^{(m)}=\widetilde{X}_{j}\left(\widetilde{X}_{j}^{\top} A_{x_{j}}^{(m)} \widetilde{X}_{j}\right)^{-1} \widetilde{X}_{j}^{\top} A_{x_{j}}^{(m)}
$$

onde a $i$-ésima linha de $\tilde{X}_{j}$ é $\left(1, x_{i j}, x_{i j}^{2}, \ldots, x_{i j}^{d}\right)$ e $A_{x_{j}}^{(m)}=U_{x_{j}} W^{(m)}$, com $U_{x_{j}}$ e $W^{(m)}$ dados por (3.8) e (3.15), respectivamente.

Mais uma vez podemos dizer que o retroajuste é um método eficiente para resolver o sistema (3.16), pois apresenta economia computacional (principalmente quando o número de parâmetros é grande) em relação à solução direta, que nesse caso seria dada por

$$
\left(\begin{array}{c}
\widehat{f_{2}} \\
\widehat{f_{3}} \\
\vdots \\
\widehat{f}_{p}
\end{array}\right)=\left(\begin{array}{cccc}
I & S_{2} & \ldots & S_{2} \\
S_{3} & I & \ldots & S_{3} \\
\vdots & \vdots & \ddots & \vdots \\
S_{p} & S_{p} & \ldots & I
\end{array}\right)^{-1}\left(\begin{array}{c}
S_{2} \\
S_{3} \\
\vdots \\
S_{p}
\end{array}\right) z=M^{-1} C z
$$

se a inversa de $M$ existe, onde $M$ e $C$ representam as matrizes de dimensão $n(p-1) \times n(p-1)$ e $n(p-1) \times n$, respectivamente, compostas de matrizes alisadoras centralizadas ponderadas por $W$. Como comentado na seção anterior, essa solução é útil para obter expressões simplificadas para as funções $\widehat{f}_{j}$, pois a partir de (3.17) temos que a matriz que produz $\widehat{f}_{j}$ a partir de $z$ pode ser definida como

$$
G_{j}=E_{j} \bar{M}^{-1} C, \text { para } j=2, \ldots, p,
$$

onde $E_{j}$ é uma matriz de dimensão $n \times n(p-1)$ composta por $p$ blocos de dimensão $n \times n$, sendo o $j$-ésimo bloco a matriz identidade, e os demais elementos iguais a 
zero, de tal maneira que

$$
\widehat{f_{j}}=G_{j} z, \text { para } j=2, \ldots, p .
$$

Seja $f=f_{2}+\ldots+f_{p}$ a parte não paramétrica do modelo aditivo generalizado, então temos que

$$
\widehat{f}=G_{2} z+\ldots+G_{p} z=G_{N P} z
$$

onde $G_{N P}=G_{2}+\ldots+G_{p}$ é a matriz suavizadora ponderada que produz $\widehat{f}$ a partir de $z$. Assim, o vetor de estimativas do preditor dado em (3.14) pode ser escrito como

$$
\widehat{\eta}=1 \widehat{\beta_{1}}+\widehat{f}=1 \bar{z}+G_{2} z+\ldots+G_{p} z=G_{1} z+G_{2} z+\ldots+G_{p} z=G z
$$

onde aqui 1 representa o vetor $n \times 1$ de uns, $G=G_{1}+G_{2}+\ldots+G_{p}$ e $G_{1}=11^{\top} / n$.

Note que para os MAG's temos que $\eta=G z$, mas $G$ não é um operador linear pela sua dependência com $\widehat{\mu}$, e logo $y$, através dos pesos $W$, que são considerados nas matrizes alisadoras $S_{i}$, como já comentado anteriormente. Como as matrizes $G_{j}$ dependem de $y$ através de $W$, esse peso deve ser considerado no procedimento de obtenção das matrizes $G_{j}$, para $j=2, \ldots, p$. A idéia é exatamente a mesma descrita na Seção 3.3.1, mas aqui os $n$ ajustes de modelos aditivos são ponderados com peso $W$, dado por (3.15) na convergência do processo de ajuste do MAG original (3.14), as variáveis resposta são também as colunas $e_{i}$ da matriz identidade $I$ de dimensão $n \times n$, e o preditor é sempre o mesmo do MAG original (3.14). Hastie e Tibshirani (1990) fornecem expressões mais simples de $G$ para modelos com duas funções alisadoras, onde temos que

$$
\begin{aligned}
& G_{2}=I-\left(I-S_{2} S_{3}\right)^{-1}\left(I-S_{2}\right), \\
& G_{3}=I-\left(I-S_{3} S_{2}\right)^{-1}\left(I-S_{3}\right),
\end{aligned}
$$

e nesse caso

$$
G=G_{1}+G_{N P}=G_{1}+G_{2}+G_{3}=11^{\top} / n+I-\left(I-S_{3}\right)\left(I-S_{2} S_{3}\right)^{-1}\left(I-S_{2}\right) .
$$

Considere agora o modelo aditivo generalizado semi-paramétrico

$$
\eta_{i}=g\left(\mu_{i}\right)=\sum_{j=1}^{p_{1}} \beta_{j} x_{i j}+\sum_{j=p_{1}+1}^{p} f_{j}\left(x_{i j}\right)
$$

com $x_{i 1}=1$ para todo $i=1, \ldots, n$, também já apresentado no início desse capítulo. Os parâmetros $\beta_{1}, \ldots, \beta_{p_{1}}$ e as funções $f_{p_{1}+1}, \ldots, f_{p}$ podem da mesma 
forma ser estimados pela combinação dos algoritmos de ponderação local e retroajuste seguindo os 4 passos descritos anteriormente, com o acréscimo de informações que levem em conta a parte paramétrica do modelo acima. Em analogia aos processos de estimação dos MLG's e dos MAG's apresentados anteriormente, podemos dizer que o ajuste do MAG semi-paramétrico (3.19) está condicionado à solução das seguintes equações

$$
\beta=\left(X_{P}^{\top} W X_{P}\right)^{-1} X_{P}^{\top} W\left(z-\sum_{j=p_{1}+1}^{p} f_{j}\right)
$$

e

$$
f_{j}=S_{j}\left(z-X_{P} \beta-\sum_{k \neq j} f_{k}\right), \text { para } j=p_{1}+1, \ldots, p
$$

onde $\beta=\left(\beta_{1}, \ldots, \beta_{p_{1}}\right)^{\top}, X_{P}$ é a matriz de covariadas da parte paramétrica do modelo, ou seja, é a matriz de linhas $x_{i}^{\top}=\left(1, x_{i 2}, \ldots, x_{i p_{1}}\right)$, e as matrizes $S_{j}$ são as matrizes alisadoras centralizadas ponderadas. Este procedimento é análogo ao de um modelo não paramétrico com $p$ alisadores, onde um alisador é o operador projeção $S_{1}=X_{P}\left(X_{P}^{\top} W X_{P}\right)^{-1} X_{P}^{\top} W$, que produz o valor $X_{P} \widehat{\beta}$, e os $\left(p-p_{1}\right)$ alisadores restantes produzirão $f_{p_{1}+1}, \ldots, f_{p}$. Assim, o ajuste desse modelo pode ser obtido iterativamente da seguinte forma

1. Inicialize o processo com

$$
\beta^{(0)}=\left(\beta_{1}^{(0)}, \ldots, \beta_{p_{1}}^{(0)}\right)^{\top} \quad \text { e } \quad f_{p_{1}+1}^{(0)}=\ldots=f_{p}^{(0)}=0, \text { para } m=0
$$

$\operatorname{com} \beta_{i}^{(0)}=g(\bar{y})$, por exemplo.

2. Considere $m=m+1$ e calcule a variável dependente modificada $z^{(m)}$ e a matriz de pesos $W^{(m)}$ da seguinte forma

$$
z_{i}^{(m)}=\eta_{i}^{(m-1)}+\left(y_{i}-\mu_{i}^{(m-1)}\right)\left(\frac{\partial \eta_{i}}{\partial \mu_{i}}\right)_{(m-1)},
$$

onde

$$
\begin{gathered}
\eta_{i}^{(m-1)}=X_{P} \beta^{(m-1)}+\sum_{j=p_{1}+1}^{p} f_{j}^{(m-1)}\left(x_{i j}\right), \\
\mu_{i}^{(m-1)}=g^{-1}\left(\eta_{i}^{(m-1)}\right)
\end{gathered}
$$

e

$$
W^{(m)}=\operatorname{diag}\left(w_{1}^{(m)}, \ldots, w_{n}^{(m)}\right)
$$


com

$$
w_{i}^{(m)}=\left(\frac{\partial \mu_{i}}{\partial \eta_{i}}\right)_{(m-1)}^{2} \frac{1}{V\left(\mu_{i}^{(m-1)}\right)}
$$

onde $V(\cdot)$ é a função de variância apresentada na Seção 2.1. Em seguida calcule as estimativas do vetor de parâmetros do modelo

$$
\beta^{(m)}=\left(X_{P}^{\top} W^{(m)} X_{P}\right)^{-1} X_{P}^{\top} W^{(m)}\left(z^{(m)}-\sum_{j=p_{1}+1}^{p} f_{j}^{(m-1)}\right) .
$$

3. Para obter $f_{p_{1}+1}^{(m)}, \ldots, f_{p}^{(m)}$, ajuste um modelo aditivo ponderado com variável dependente $\left(z^{(m)}-X_{P} \beta^{(m)}\right)$ e peso $W^{(m)}$ através do algoritmo de retroajuste. Ou seja, calcule seqüencialmente para $j=p_{1}+1, \ldots, p, p_{1}+1, \ldots, p$,

$$
f_{j}=S_{j}^{(m)}\left(z^{(m)}-X_{P} \beta^{(m)}-\sum_{k \neq j} f_{k}\right),
$$

até que cada função individual convirja obtendo assim $f_{p_{1}+1}^{(m)}, \ldots, f_{p}^{(m)}$, onde $S_{j}^{(m)}, j=p_{1}+1 \ldots, p$, são as matrizes alisadoras centralizadas ponderadas por $W^{(m)}$.

4. Para um valor de $\epsilon$ pré-estabelecido, repita os passos 2 e 3 até que

$$
\frac{\sum_{j=p_{1}+1}^{p}\left\|f_{j}^{(m)}-f_{j}^{(m-1)}\right\|}{\sum_{j=p_{1}+1}^{p}\left\|f_{j}^{(m-1)}\right\|} \leq \epsilon
$$

$\mathrm{e}$

$$
\frac{\left\|\beta^{(m)}-\beta^{(m-1)}\right\|}{\left\|\beta^{(m-1)}\right\|} \leq \epsilon
$$

ou

$$
\sum_{j=1}^{n}\left|D_{p}\left(y_{i}, \mu_{i}^{(m)}\right)-D_{p}\left(y_{i}, \mu_{i}^{(m-1)}\right)\right| \leq \epsilon .
$$

De forma similar à apresentada no sistema de equações (3.17), a partir de (3.21) podemos escrever que

$$
f_{j}=M^{-1} C\left(z-X_{P} \beta\right)=\widetilde{G}_{j}\left(z-X_{P} \beta\right)=G_{j} z, \text { para } j=p_{1}+1, \ldots, p,
$$

onde $M$ e $C$ representam as matrizes de dimensão $n\left(p-p_{1}\right) \times n\left(p-p_{1}\right)$ e $n\left(p-p_{1}\right) \times$ $n$, respectivamente, compostas de matrizes alisadoras centralizadas ponderadas por $W$. No entanto, no caso dos modelos aditivos semi-paramétricos as matrizes $G_{j}$ não apresentam forma explícita, o que na prática dificulta ainda mais sua obtenção e o estudo das propriedades das funções não paramétricas $f_{j}$. 
Seja $f=f_{p_{1}+1}+\ldots+f_{p}$, temos então que

$$
f=G_{p_{1}+1} z+\ldots+G_{p} z=G_{N P} z
$$

ou

$$
f=\widetilde{G}_{p_{1}+1}\left(z-X_{P} \beta\right)+\ldots+\widetilde{G}_{p}\left(z-X_{P} \beta\right)=\widetilde{G}_{N P}\left(z-X_{P} \beta\right),
$$

onde $G_{N P}=G_{p_{1}+1}+\ldots+G_{p}$ é a matriz que produz $f$ a partir de $z$, e $\widetilde{G}_{N P}=$ $\widetilde{G}_{p_{1}+1}+\ldots+\widetilde{G}_{p}$ é a matriz que produz $f$ a partir de $z-X_{P} \beta$.

Hastie e Tibshirani (1990, Seção 6.7) e Thurston, Wand e Wiencke (2000) apresentam uma solução direta para as equações (3.20) e (3.21) que evita a aplicação do algoritmo de retroajuste. Para isso $f$ em (3.20) é substituida por $\widetilde{G}_{N P}(z-X \widehat{\beta})$, dada em (3.22), que fornece

$$
\widehat{\beta}^{(m)}=\left\{\left(X_{P}^{\top} \widehat{W}^{(m-1)}\left(I-\widetilde{G}_{N P}^{(m-1)}\right) X_{P}\right)\right\}^{-1} X_{P}^{\top} \widehat{W}^{(m-1)}\left(I-\widetilde{G}_{N P}^{(m-1)}\right) z^{(m-1)} .
$$

Aplicando somente o algoritmo de ponderação local (passo 2 do esquema descrito anteriormente) obtemos novos valores para $\widehat{\eta}^{(m)}, \widehat{\mu}^{(m)}, z^{(m)}$ e $\widehat{W}^{(m)}$. Então, após obter essas estimativas, uma nova estimativa $\widehat{f}^{(m)}=\widetilde{G}_{N P}^{(m)}\left(z-X \widehat{\beta}^{(m)}\right)$ é obtida a partir de (3.22). Esse processo deve ser repetido até a convergência.

Assim, o vetor de estimativas do preditor dado em (3.19) pode ser escrito como

$$
\widehat{\eta}=X_{P} \widehat{\beta}+\widehat{f}=X_{P} \widehat{\beta}+\widetilde{G}_{N P}\left(z-X_{P} \widehat{\beta}\right)=G_{P} z+G_{N P} z=G z .
$$

onde, pela expressão de $\widehat{\beta}$ dada em (3.23), temos que a matriz que produz a parte paramétrica do modelo (3.19) é dada por

$$
G_{P}=X_{P}\left(X_{P}^{\top} W\left(I-\widetilde{G}_{N P}\right) X_{P}\right)^{-1} X_{P}^{\top} W\left(I-\widetilde{G}_{N P}\right) .
$$

Evidentemente que nos MAG's semi-paramétricos também temos que $G$ depende de $y$, como já comentado anteriormente para os MAG's não paramétricos.

No entanto, a obtenção das matrizes alisadoras ponderadas $\widetilde{G}_{N P}=\widetilde{G}_{p_{1}+1}+$ $\ldots+\widetilde{G}_{p}$, e principalmente de $G_{N P}=G_{p_{1}+1}+\ldots+G_{p}$, apresenta um custo computacional muito elevado. No caso da presença de apenas um termo não paramétrico no modelo, podemos utilizar o resultado apresentado por Hastie e Tibshirani (1990), válido para duas funções alisadoras e já apresentado anteriormente, onde aqui $S_{1}$ seria a matriz $X_{P}\left(X_{P}^{\top} W X_{P}\right)^{-1} X_{P}^{\top} W$ que vai produzir $X_{P} \widehat{\beta}$ e $S_{2}$ a matriz alisadora centralizada ponderada que vai produzir a função não paramétrica do modelo $\hat{f}$. Nesse caso temos que

$$
\begin{aligned}
& G_{1}=I-\left(I-S_{1} S_{2}\right)^{-1}\left(I-S_{1}\right), \\
& G_{2}=I-\left(I-S_{2} S_{1}\right)^{-1}\left(I-S_{2}\right),
\end{aligned}
$$


e logo,

$$
G=G_{1}+G_{2}=I-\left(I-S_{2}\right)\left(I-S_{1} S_{2}\right)^{-1}\left(I-S_{1}\right) .
$$

Para os demais casos, Opsomer (2000) deduz expressões recursivas para a obtenção de $G_{j}, j=p_{1}, \ldots, p$, que apresentam um custo computacional muito elevado. Por outro lado, esse cálculo pode ser realizado seguindo a mesma idéia apresentada para o MAG não paramétrico, dado em (3.14), ou seja, podemos obtê-las também pelo ajuste de $n$ modelos aditivos ponderados com peso $W$, dado por (3.15) na convergência do processo de ajuste do MAG original (3.19), as variáveis resposta são também as colunas $e_{i}$ da matriz identidade $I$ de dimensão $n \times n$, e o preditor é o mesmo do MAG original (3.19). O cálculo das matrizes $\widetilde{G}_{j}, j=p_{1}, \ldots, p$, pode ser realizado dessa mesma forma, mas aqui o preditor dos $n$ modelos aditivos ponderados com peso $W$, é somente a parte não paramétrica do MAG original apresentado em (3.19).

A convergência do procedimento de ajuste dos MAG's de uma forma geral está condicionada à convergência do retroajuste, uma vez que, como nos MLG's, o procedimento de ponderação local não apresenta problemas de convergência. Buja et al. (1989) e Opsomer e Ruppert (1999) apresentam condições sob as quais o algoritmo de retroajuste converge, e de acordo com essa teoria, o maior problema está relacionado à existência de dependência não linear entre covariáveis do modelo aditivo, um fenômeno análogo à multicolinearidade, e conhecido como concurvidade. Hastie e Tibshirani (1990, Seção 5.4.2) propõem o ajuste de um algoritmo de retroajuste modificado que é mais eficiente quando as covariáveis são correlacionadas. No entanto, este procedimento não tem sido explorado na literatura.

Para avaliar a necessidade da aplicação de um MAG ao invés de um MLG no estudo da associação entre variáveis, a construção de gráficos de dispersão do preditor $\eta=g(y)$ contra as covariáveis pode ser muito útil, até para a escolha do parâmetro de suavização no caso em que a relação entre essas variáveis não apresentar uma forma definida, ou seja, quando assumirmos uma função não paramétrica para o estudo da relação entre $g(y)$ e $x$.

O comando gam do software S-Plus realiza o ajuste de modelos aditivos generalizados, logicamente, para as distribuições que pertencem à família exponencial, com diferentes funções de ligação.

Exemplo 3.1: Considerando o conjunto de dados simulados apresentado na Figura 3.1, vamos assumir a distribuição de Poisson para $y$ e considerar a função de ligação logarítmica. Nesse caso temos a função de ligação $g\left(\mu_{i}\right)=\log \mu_{i}=\eta_{i}$ e a função de variância $V\left(\mu_{i}\right)=\mu_{i}$. Logo, os pesos são dados por $w_{i}=\mu_{i}$, e a 
variável dependente modificada será

$$
z_{i}=\log \mu_{i}+\frac{\left(y_{i}-\mu_{i}\right)}{\mu_{i}} .
$$

O gráfico de $x$ contra $\log (y)$ está apresentado na Figura 3.7, onde observamos mais uma vez que assumir uma relação linear entre $x$ e $\log (y)$ não é adequado. Assim, propomos o ajuste do seguinte MAG com distribuição de Poisson

$$
\mathrm{E}\left(Y_{i}\right)=\mu_{i}=\exp \left(\beta_{1}+\text { loess }\left(x_{i}\right)\right)
$$

com os parâmetros da função loess dados por $\lambda=30 \%$ e $d=2$. Esse ajuste foi realizado com o auxílio do S-Plus pelo comando gam ( $\mathrm{y} \sim \mathrm{lo}(\mathrm{x}, \mathrm{span}=0.30, \mathrm{~d}=2$ ), data=dados, family=poisson). Os valores ajustados estão apresentados em azul na Figura 3.8.

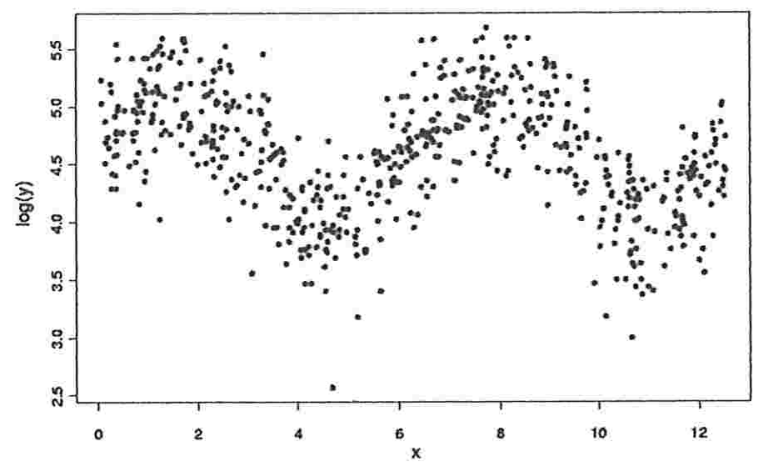

Figura 3.7: Diagrama de dispersão do logaritmo de $y$ contra $x$.

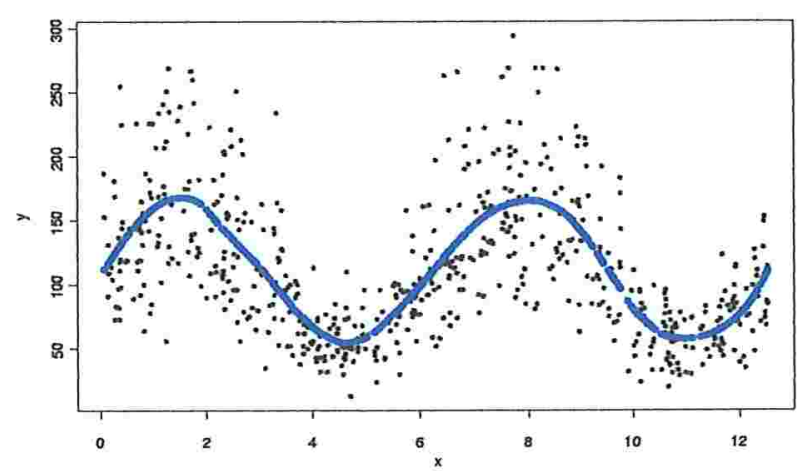

Figura 3.8: Valores ajustados pelo MAG com distribuição de Poisson. 


\subsection{Análise Inferencial}

Os testes de hipóteses para avaliar a importância de cada covariada no ajuste de um MAG, assim como nos MLG's, são baseados no desvio do modelo $S_{p}$, definido em (2.6), lembrando que aqui temos

$$
\widehat{\mu}_{i}=g^{-1}\left(\widehat{\eta}_{i}\right)=g^{-1}\left(\widehat{\beta}_{1}+\sum_{j=2}^{p} \widehat{f}_{j}\left(x_{i j}\right)\right),
$$

ou

$$
\widehat{\mu}_{i}=g^{-1}\left(\widehat{\eta}_{i}\right)=g^{-1}\left(\sum_{j=1}^{p_{1}} \widehat{\beta}_{j} x_{i j}+\sum_{j=p_{1}+1}^{p} \widehat{f}_{j}\left(x_{i j}\right)\right) .
$$

E como apresentado em (3.18) e (3.24), na convergência do processo de estimação dos MAG's, temos também que

$$
\widehat{\eta}=G z
$$

Apesar de nem mesmo a distribuição assintótica do desvio dos MAG's ter sido determinada, essa medida também pode ser utilizada informalmente para avaliar a qualidade do ajuste do modelo. Hastie e Tibshirani (1990) mostraram através de simulações que a distribuição qui-quadrado com graus de liberdade

$$
\mathrm{gl}=n-\operatorname{tr}\left(2 G-G^{\top} W G W^{-1}\right)
$$

é uma boa aproximação para sua distribuição, lembrando que $W$ é a matriz de pesos associada ao ajuste dos MAG's. Os cálculos para a obtenção dos graus de liberdade definidos acima apresentam elevado custo computacional por envolver a matriz alisadora ponderada $G$. Assim, uma aproximação para os graus de liberdade do desvio pode ser dada por

$$
\mathrm{gl}=n-1-\sum_{i=1}^{p}\left(\operatorname{tr}\left(S_{j}\right)-1\right),
$$

lembrando que as matrizes $S_{j}$ representam matrizes alisadoras centralizadas ponderadas associadas a cada covariada (ver Hastie e Tibshirani, 1990, Seção 6.8.3).

Agora suponha que $G_{1}$ e $G_{2}$ sejam matrizes operadoras do ajuste dos MAG's que produzem $\widehat{\eta}_{1}=G_{1} z_{1}$ com $q$ covariadas e $\widehat{\eta}_{2}=G_{2} z_{2}$ com $p>q$ covariadas, respectivamente, sendo

$$
\widehat{\eta}_{2}=\widehat{\eta}_{1}+\widehat{\eta}^{\prime}
$$

onde $\widehat{\eta}^{\prime}$ é o preditor que contém $(p-q)$ covariadas. Se o parâmetro de dispersão $\phi$ da família exponencial for conhecido, o teste do desvio parcial, apresentado na Seção 2.4, pode da mesma forma ser realizado para avaliar a contribuição 
das $p-q$ covariadas presentes somente em $\eta_{2}$, ou seja, a contribuição de $\eta^{\prime}$ no ajuste do MAG. Nesse caso Hastie e Tibshirani (1990, Seção 6.8.3) mostram que podemos aproximar a distribuição do desvio parcial $D_{1}-D_{2}$ por uma distribuição qui-quadrado com graus de liberdade dados por

$$
\mathrm{gl}_{\widehat{\eta}_{2}}-\mathrm{gl}_{\widehat{\eta}_{1}}=\operatorname{tr}\left(2 G_{1}-G_{1}^{\top} W_{1} G_{1} W_{1}^{-1}\right)-\operatorname{tr}\left(2 G_{2}-G_{2}^{\top} W_{2} G_{2} W_{2}^{-1}\right) .
$$

Quando o parâmetro de dispersão $\phi$ da família exponencial for desconhecido, um teste $F$ aproximado é mais apropriado para avaliar a contribuição de $\eta^{\prime}$. Por analogia ao modelo Gaussiano, onde o desvio é igual à soma de quadrados dos resíduos, temos a seguinte aproximação assintótica para a estatística desse teste

$$
F=\frac{\left(D_{1}-D_{2}\right) /\left(\mathrm{gl}_{\hat{\eta}_{2}}-\mathrm{gl}_{\widehat{\eta}_{1}}\right)}{D_{2} /\left(n-\mathrm{gl}_{\widehat{\eta}_{2}}\right)} \sim F_{\mathrm{g}_{\hat{\eta}_{2}}-\mathrm{gl}_{\hat{\eta}_{1}}, n-\mathrm{gl}_{\hat{l}_{2}}},
$$

ver Hastie e Tibshirani (1990, Seções 6.8.1 e 3.9) para maiores detalhes. Chambers e Hastie (1993) utilizam esse teste para avaliar se a melhor contribuição de cada covariada no ajuste do modelo se dá de forma linear ou não paramétrica, ou seja, para uma determinada covariada $X_{j}$, comparam o ajuste do modelo com parâmetro linear $\widehat{\beta}_{j}$ com o ajuste de outro modelo com a função não paramétrica $\widehat{f_{j}}$, através da estatística $F$ aproximada apresentada acima.

A matriz de variância e covariância assintótica de $\widehat{\eta}=G z$ pode ser obtida a partir da aproximação da variável dependente $z$ por uma quantidade assintoticamente equivalente $z_{0}$, de média $\eta_{0}$ e matriz de variância e covariância $\phi W_{0}^{-1}$. Como a matriz $G$ não é um operador linear devido à sua dependência em $y$ através de $W$, devemos também considerar sua versão assintótica $G_{0}$. Logo, temos que

$$
\operatorname{Var}(\widehat{\eta})=\operatorname{Var}(G z) \approx \operatorname{Var}\left(G z_{0}\right) \approx \phi G_{0} W_{0}^{-1} G_{0}^{\top} \approx \phi G W^{-1} G^{\top} .
$$

Similarmente,

$$
\operatorname{Var}\left(\widehat{f_{j}}\right) \approx \phi G_{j} W^{-1} G_{j}^{\top},
$$

lembrando que $G_{j}$ é a matrix que produz $\widehat{f}_{j}$ a partir de $z$.

Assumindo válidas as condições de regularidade usuais, inclusive consistência, pode-se mostrar que $\widehat{\eta}$ tem distribuição assintótica $\mathrm{N}\left(\eta_{0}, \phi G_{0} W_{0}^{-1} G_{0}\right.$ ) (Hastie e Tibshirani, 1990, Seção 3.8.2).

Thurston, Wand e Wiencke (2000) apresentam uma aproximação para a matriz de variância e covariância de $\widehat{\beta}$, que é dada por

$$
\widehat{\operatorname{Var}(\widehat{\beta})}=\left(X_{P}^{\top} W\left(I-\widetilde{G}_{N P}\right) X_{P}\right)^{-1} \text {. }
$$

Essa variância é exata somente se a matriz alisadora ponderada $G_{N P}$, que produz a parte não paramétrica do modelo, puder ser escrita como o produto de uma 
matriz alisadora $S$ com uma matriz de pesos $W$, sendo $S$ uma matriz simétrica e idempotente.

Apesar da relação entre as covariadas e a variável resposta apresentar forma não paramétrica, assim como nos modelos de regressão linear, podemos examinar o efeito de cada uma das covariadas sobre a resposta, condicionando esses resultar dos à presença das demais covariadas. Assim, gráficos de $\widehat{f_{j}}$ contra $x_{i j}$, para todo $i$, podem ser úteis para a interpretação dos resultados do modelo, que no caso, correspondem à intepretação dos parâmetros $\beta$ no modelo de regressão linear. Uma aproximação do intervalo de confiança para essa curva com coeficiente de confiança igual a $\gamma=1-\alpha$ pode ser obtida a partir de (3.29), e é dada por

$$
\widehat{f_{j}} \mp z_{\alpha / 2} \operatorname{diag}\left(\phi G_{j} W^{-1} G_{j}^{\top}\right)^{1 / 2} \text {. }
$$

onde $z_{\alpha}$ é o quantil de ordem $1-\alpha$ de distribuição normal padrão.

Hastie e Tibshirani $(1990,1987)$ apresentam a análise inferencial dos MAG's de uma forma mais detalhada.

Exemplo 3.1 (continuação): O desvio do modelo de Poisson (3.25), ajustado na seção anterior, é igual a 7101,43 com 589,08 graus de liberdade, indicando que esse ajuste não parece adequado. Pelo teste do desvio parcial, concluímos que a contribuição de $x$ no modelo (3.25) é significativa $(p<0,001)$. O teste F para comparação do modelo não paramétrico dado em (3.25) com o modelo de regressão linear de Poisson $\mu_{i}=\exp \left(\beta_{0}+\beta_{1} x\right)$ apresenta estatística igual a $71,96$ ( $p<0,001)$, indicando que a associação entre $x$ e $y$ é melhor representada por uma função não paramétrica, como esperado pela Figura 3.1. A função não paramétrica estimada e respectiva banda de confiança estão representadas na Figura 3.9. As linhas pontilhadas são uma aproximação para o intervalo de confiança da curva de alisamento ajustada, e as barras verticais no eixo das abscissas representam as observações, ou seja, os valores de $x$ efetivamente observados na amostra.

\subsection{Seleção dos Parâmetros de Alisamento}

A seleção do parâmetro de alisamento $\lambda_{j}$ de cada $f_{j}$ é um passo muito importante no ajuste de um MAG, pois existe uma forte dependência entre a seleção de MAG's e a seleção dos parâmetros de alisamento. E assim como já comentado na 


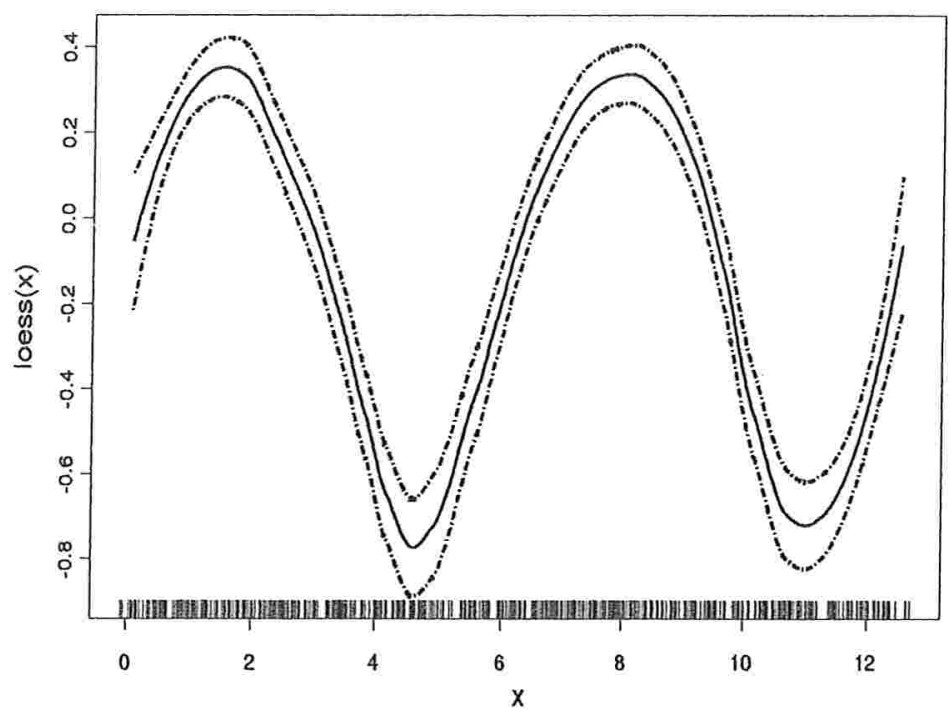

Figura 3.9: Gráfico da função loess estimada com $\lambda=30 \%$ e $d=2$ no modelo de regressão de Poisson.

Seção 3.2, o parâmetro $\lambda$ de um alisador é de grande importância para sua aplicação.

Consideremos, por exemplo, um modelo com $p$ termos não paramétricos. Neste caso, temos que escolher os parâmetros $\lambda_{1}, \ldots, \lambda_{p}$ das funções $f_{1}, \ldots, f_{p}$. Se considerarmos como critério de escolha dos $\lambda$ 's simplesmente a minimização da função desvio $D(y, \mu)$, chegaremos ao desvio nulo com $\widehat{\mu}_{i}=y_{i}$, para todo $i$. Podemos evitar esse problema utilizando o método de validação cruzada, descrito na Seção 3.2, aplicado ao desvio do modelo, ou seja, aqui temos

$$
V C_{D}\left(\lambda_{1}, \ldots, \lambda_{p}\right)=\frac{1}{n} \sum_{i=1}^{n} D\left(y_{i}, \widehat{\mu}^{-(i)}\right),
$$

onde $\widehat{\mu}^{-(i)}$ é o valor ajustado para a $i$-ésima observação quando o ponto $\left(x_{i}, y_{i}\right)$ é omitido na amostra. A idéia é encontrar os valores de $\lambda_{1}, \ldots, \lambda_{p}$ que minimizam $V C_{D}$, mas esse procedimento apresenta um custo computacional muito elevado, uma vez que necessita de $n$ ajustes do MAG sob investigação para cada conjunto de valores pré-fixados para $\lambda_{1}, \ldots, \lambda_{p}$.

De modo alternativo, podemos utilizar também a estatística baseada na informação de Akaike, já apresentada na Seção 2.4, que para os MAG's apresenta a seguinte definição

$$
A I C=\frac{1}{n} \sum_{i=1}^{n} D\left(y_{i}, \widehat{\mu}_{i}\right)+\frac{2 \phi \operatorname{tr}(G)}{n} .
$$


Na prática, essa estatística é muito utilizada pela sua facilidade computacional em relação à $V C_{D}$, pois o cálculo da estatística $A I C$ para cada conjunto de valores pré-fixados $\lambda_{1}, \ldots, \lambda_{p}$ é obtido a partir de um único ajuste do MAG sob investigação.

Como o valor do parâmetro de alisamento pode afetar a autocorrelação dos resíduos (Schwartz, 1999), esse fato também pode ser utilizado como um critério na seleção desses parâmetros, buscando um modelo com $\lambda_{1}, \ldots, \lambda_{p}$ que satisfaça a suposição de independência, ou seja, que apresente autocorrelações baixas.

Como já comentado na Seção 3.2, os parâmetros de alisamento do modelo podem também ser determinados pela natureza dos dados que estão sendo avaliados.

Como não existem estudos práticos suficientes para comprovar a eficiência de um determinado método na escolha dos parâmetros de alisamento no ajuste dos MAG's, a construção de gráficos com a curva alisada sobreposta aos dados são ainda de grande importância para visualizar o grau de alisamento mais adequado para cada função alisadora. Nesse caso, essa avaliação gráfica deve ser feita em função do preditor, ou seja, de $g(y)$, para visualizar qual a melhor forma não paramétrica que representa a relação de cada covariada com a resposta.

Exemplo 3.1 (continuação): A seleção de modelos aditivos está diretamente ligada à seleção dos parâmetros de alisamento de cada função alisadora no ajuste dos MAG's. Para ilustrar esse procedimento, consideramos o ajuste dos modelos aditivos $\mu_{i}=\exp \left(\beta_{0}+\operatorname{loess}\left(x_{i}, \lambda, d\right)\right)$ com distribuição de Poisson e calculamos a função de Akaike $(A I C)$, a validação cruzada $\left(V C_{D}\right)$ e a soma dos quadrados das autocorrelações dos resíduos com defasagem de 1 a $30\left(\sum_{i=1}^{30} F A C_{i}^{2}\right)$ para um pequeno conjunto de valores de $\lambda \operatorname{com} d=1$ e $d=2$. Essas medidas estão apresentadas na Tabela 3.1. A avaliação gráfica desses parâmetros está apresentada na Figura 3.10, onde temos as estimativas do preditor de cada modelo sobrepostas aos dados, ou seja, gráficos de $\log (y)$ contra $x$ e $\widehat{\eta}$ contra $x$. Avaliando esses resultados, podemos observar que, entre os modelos estudados, o modelo com parâmetros $\lambda=15 \%$ e $d=2$ minimiza a função $A I C$ e a soma dos quadrados das autocorrelações dos resíduos com defasagem de 1 a 30 , mas pela avaliação gráfica da Figura 3.10 observamos que esse modelo apresenta exagerado ajuste aos dados (a curva estimada é pouco lisa e com grande variabilidade). O modelo com parâmetros $\lambda=30 \%$ e $d=2$ minimiza o valor de $V C_{D}$, apresenta $A I C$ relativamente baixo quando comparado aos demais e graficamente parece reproduzir muito bem a tendência de $y$ em relação a $x$. Vale destacar que a soma dos quadrados das autocorrelações dos resíduos com defasagem de 1 a 30 apresenta pequena variabilidade entre os modelos estudados. Assim, de uma forma geral, podemos 
concluir que o modelo com parâmetros $\lambda=30 \%$ e $d=2$ parece adequado para a análise desses dados.

Tabela 3.1: Cálculo da função de Akaike $(A I C)$, da validação cruzada $\left(V C_{D}\right)$ e da soma dos quadrados das autocorrelações dos resíduos com defasagem de 1 a $30\left(\sum_{i=1}^{30} F A C_{i}^{2}\right)$ para um pequeno conjunto de valores de $\lambda \operatorname{com} d=1$ e $d=2$ no ajuste de modelos aditivos com distribuição de Poisson.

\begin{tabular}{ccccc}
\hline$\lambda$ & $d$ & $A I C$ & $\sum_{i=1}^{30} F A C_{i}^{2}$ & $V C_{D}$ \\
\hline $15 \%$ & 1 & 11,845 & 1,045 & 12,343 \\
$30 \%$ & 1 & 13,126 & 1,044 & 13,416 \\
$45 \%$ & 1 & 15,962 & 1,045 & 16,216 \\
$60 \%$ & 1 & 18,273 & 1,043 & 18,492 \\
$15 \%$ & 2 & 11,620 & 1,043 & 12,435 \\
$30 \%$ & 2 & 11,882 & 1,046 & 12,308 \\
$45 \%$ & 2 & 12,484 & 1,046 & 12,790 \\
$60 \%$ & 2 & 14,572 & 1,048 & 14,851 \\
\hline
\end{tabular}

\subsection{Técnicas de Diagnóstico}

As técnicas de diagnóstico para os MAG's são bastante similares às técnicas apresentadas na Seção 2.5, para os MLG's.

Como comentado na Seção 3.4, o desvio do modelo é também uma medida informal de qualidade de ajuste dos MAG's, de forma que valores grandes do desvio quando comparados aos seus graus de liberdade indicam que o modelo ajustado não está adequado.

Os resíduos são, da mesma forma, medidas de grande importância para a verificação das suposições do modelo, e os mais utilizados são os resíduos componentes do desvio e os resíduos de Pearson. Assim, podemos dizer que as definições apresentadas na Seção 2.5 são válidas para os MAG's, lembrando que aqui $\widehat{\mu}_{i}$ é dado por (3.26) ou (3.27):

Em geral, a análise gráfica dos resíduos do modelo ajustado é de grande importância, destacando algumas sugestões já comentadas na Seção 2.5: 

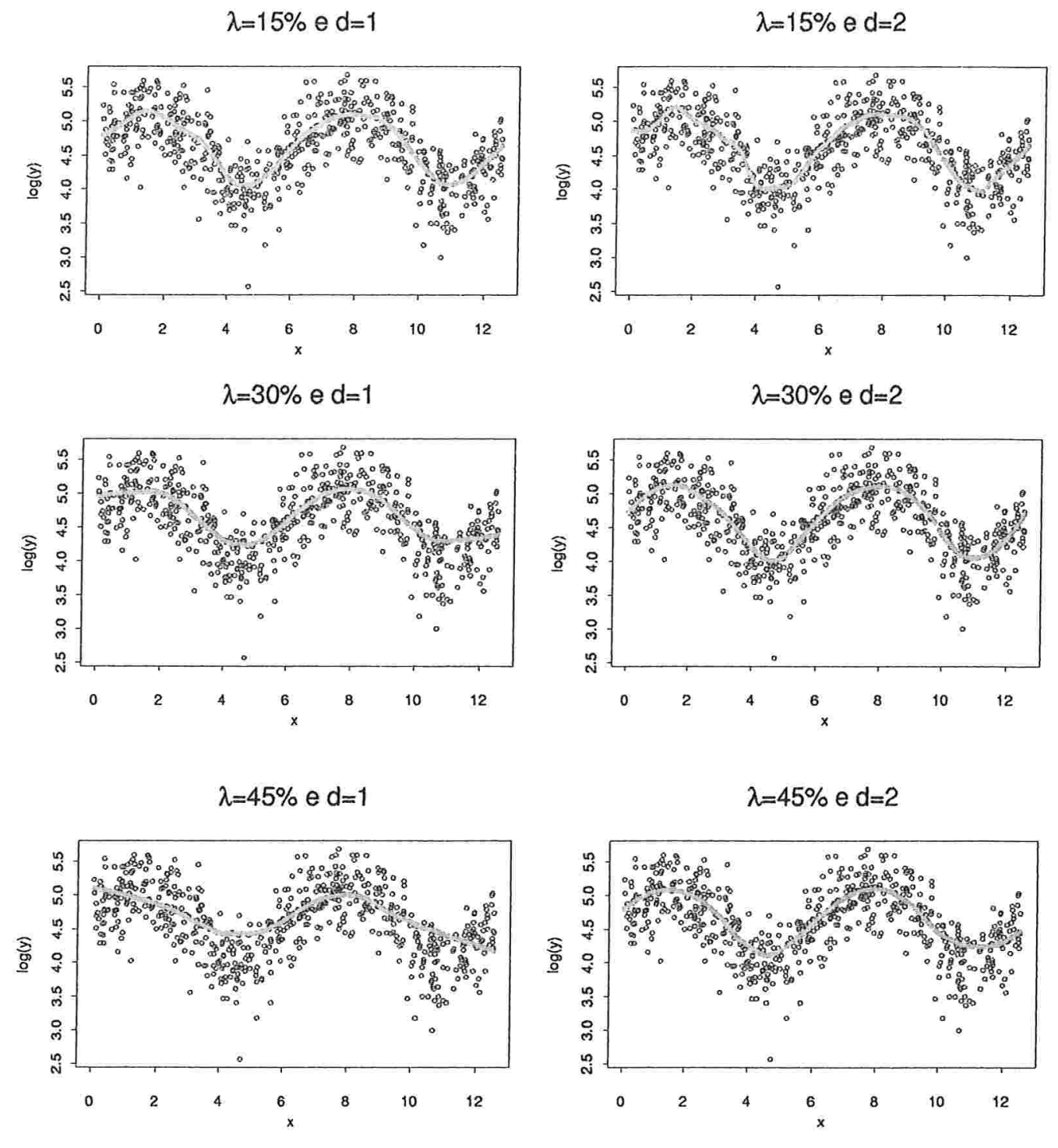

$\lambda=60 \%$ e $d=1$
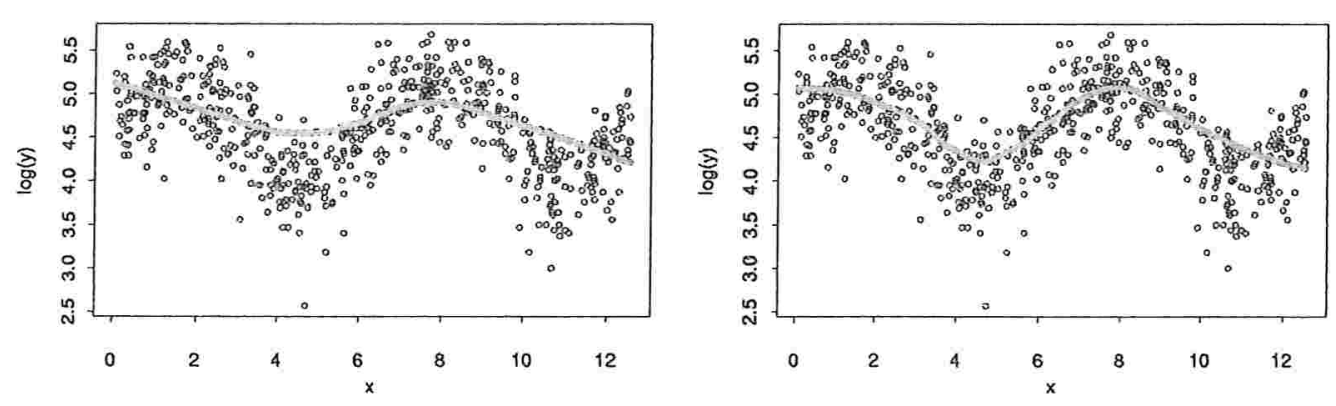

Figura 3.10: Estimativas do preditor do modelo aditivo de Poisson com curvas alisadas do tipo loess com diferentes valores de $\lambda$ e $d$, sobrepostas aos dados. 
- gráfico de probabilidade meio normal com envelope simulado para os resíduos componentes do desvio, onde espera-se que, se o modelo ajustado estiver adequado, a grande maioria dos pontos esteja dentro da banda simulada;

- gráfico de valores observados contra valores ajustados, onde espera-se que os pontos estejam próximos da reta $x=y$;

- gráfico dos resíduos componentes do desvio e resíduos de Pearson contra alguma função dos valores ajustados, como por exemplo contra o preditor linear $\widehat{\eta}_{i}$ ou mesmo seu índice $(i)$, onde espera-se uma distribuição aleatória dos resíduos em torno do eixo zero;

- gráfico da função de autocorrelação dos resíduos, onde esperam-se baixos valores das autocorrelações para todas as defaseagens,

entre outros.

Uma desvantagem dos MAG's é que resultados sobre a análise de diagnóstico específica para funções não paramétricas são muito raros na literatura. Recentemente alguns trabalhos estão sendo publicados com medidas de influência para algumas funções alisadoras aplicadas em modelos aditivos, mas estes não tratam do caso generalizado. Kim et. al (2002) apresentam esse estudo para o modelo semi-paramétrico com apenas uma função alisadora. Esses autores destacam que a influência de uma observação em cada estimador $\widehat{\beta}, \widehat{f}$ e $\widehat{\mu}$ deve ser avaliada separadamente, com medidas adequadas à definição de cada um deles. Isso porque uma observação que é influente em $\widehat{\beta}$ pode não ser em $\widehat{f}$, e vice-versa. Também pode acontecer de nenhuma observação apresentar influência em $\widehat{\beta}$ ou $\widehat{f}$, mas sim na reposta média $\widehat{\mu}$.

Exemplo 3.1 (continuação): O gráfico de probabilidade meio normal com envelope simulado do valor absoluto dos resíduos componentes do desvio, o gráfico dos resíduos de Pearson contra os valores ajustados, o gráfico dos valores observados contra os valores ajustados e o gráfico da função de autocorrelação dos resíduos de Pearson foram construídos para verificar a qualidade do ajuste do modelo aditivo de Poisson dado em (3.25). Estes gráficos estão apresentados na Figura 3.11, onde observamos que esse modelo não parece adequado pelo fato de que todos os pontos do gráfico de probabilidade meio normal estão fora do envelope simulado. Vale destacar que esse resultado já era esperado, uma vez que, como descrito na Seção 3.1, esses dados foram gerados através da distribuição binomial negativa. 
(a)

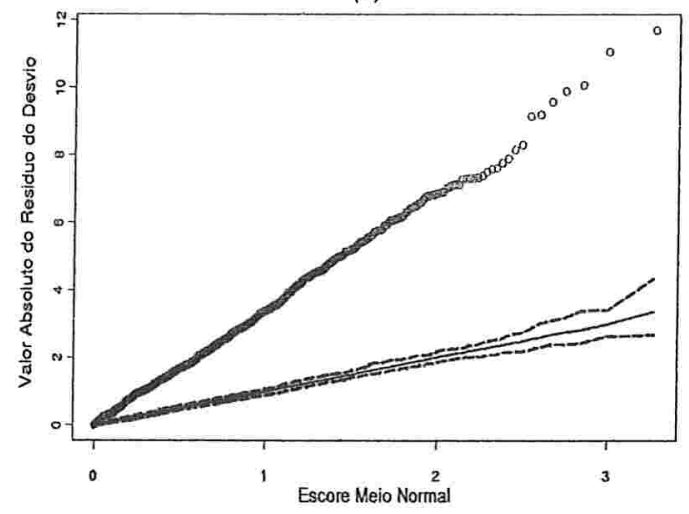

(c)

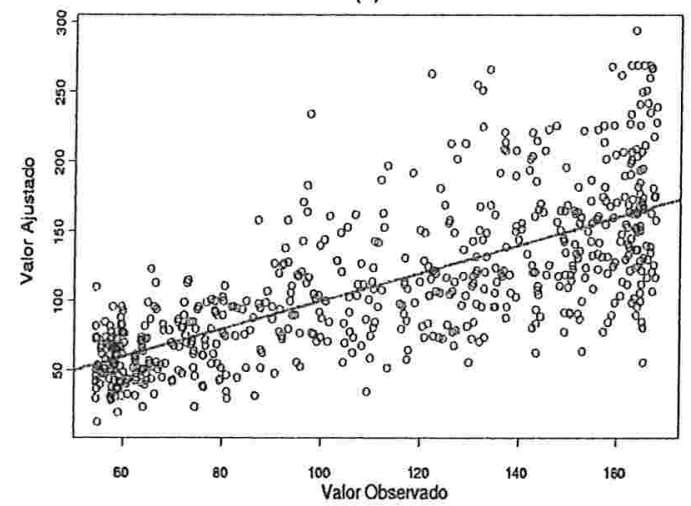

(b)

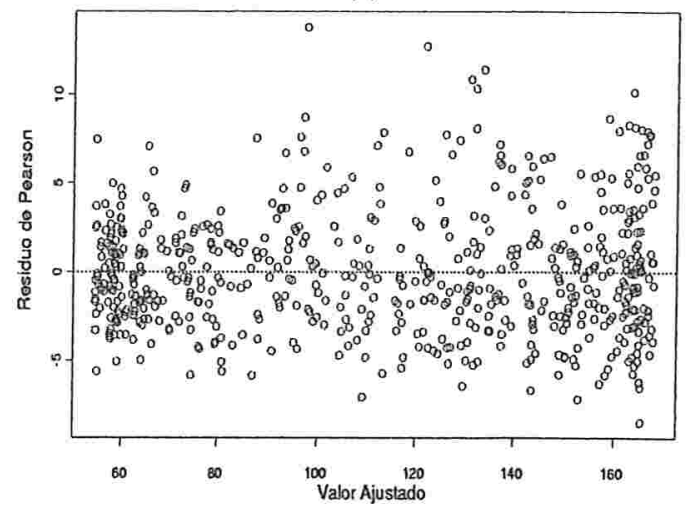

(d)

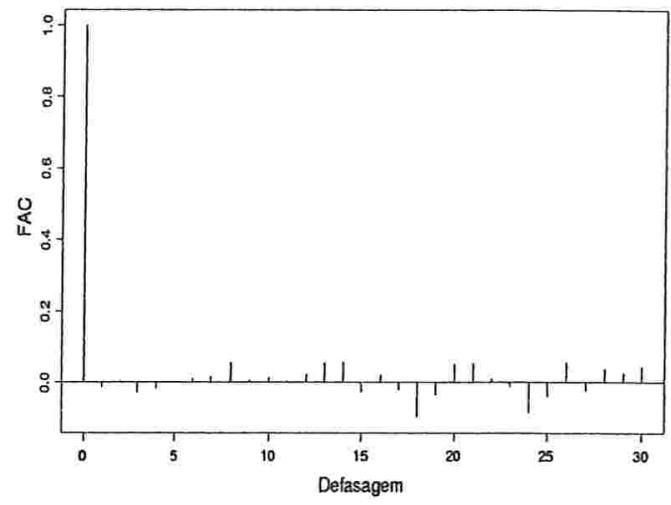

Figura 3.11: Gráfico de probabilidade meio normal com envelope simulado do valor absoluto dos resíduos componentes do desvio (a), gráfico dos resíduos de Pearson contra os valores ajustados (b), gráfico dos valores observados contra os valores ajustados (c) e gráfico da função de autocorrelação dos resíduos de Pearson (d) para o modelo aditivo de Poisson.

\subsection{Modelo Aditivo Binomial Negativo}

Sejam $Y_{1}, \ldots, Y_{n} n$ variáveis aleatórias independentes com distribuição binomial negativa de média $\mu_{i}$ e parâmetro de dispersão $k$. Se $x_{1}, \ldots, x_{n}$ são um conjunto de $n$ covariadas de dimensão $p$, suponha que a média $\mu_{i}$ de $Y_{i}$ esteja relacionada com $x_{i}$ através de (3.26) ou (3.27).

Em geral não conhecemos a distribuição da variável resposta, mas quando analisamos dados de contagem através de um MAG com distribuição de Poisson e esse ajuste não é adequado, isto pode ser devido à presença de superdispersão 
nos dados. Aqui surge a necessidade do ajuste de um MAG com distribuição binomial negativa como proposta de análise para dados superdispersos.

Como o modelo binomial negativo só pertence à família exponencial quando o parâmetro de dispersão $k$ é conhecido, Thurston, Wand e Wiencke (2000) propõem a aplicação da metodologia de análise apresentada por Hastie e Tibshirani (1990) para um determinado $k$ fixo. Reciprocamente, se o vetor de médias $\mu=\left(\mu_{1}, \ldots, \mu_{n}\right)$ é conhecido, a estimação de $k$ se reduz a um simples problema de obtenção de estimador de máxima verossimilhança de um parâmetro escalar. A expressão do logaritmo da função de verossimilhança dada em (2.14), aqui denotada por $l(k ; \mu, y)$, deve ser vista como uma função apenas de $k$, para $\mu_{i} \mathrm{e}$ $y_{i}$ fixados, $i=1, \ldots, n$, e que pode ser facilmente maximizada com a aplicação do algoritmo de Newton-Raphson, já descrito em (2.19). Os autores propõem o ajuste de $k$ e $\mu$ alternadamente até a convergência de ambos.

Dessa forma, os passos para a implementação de um algoritmo iterativo para o ajuste do modelo aditivo binomial negativo são

1. Inicialize $\widehat{k}$, por exemplo, a partir da maximização de $l(k ; \tilde{\mu}, y)$ com o auxílio do algoritmo de Newton-Raphson, onde $\tilde{\mu}$ são os valores preditos a partir de um MAG com distribuição de Poisson.

2. Ajuste um MAG com distribuição binomial negativa e parâmetro $k=\widehat{k}$, e obtenha $\widehat{\mu}$. Como nesse caso temos $k$ "conhecido", podemos considerar que a distribuição binomial negativa pertence à família exponencial (para mais detalhes ver Seção 2.6), e dessa forma o procedimento de estimação dos MAG's apresentado na Seção 3.3.2, através dos procedimentos de ponderação local e retroajuste, é válido. Lembrando que a função de variância da distribuição binomial negativa é $\mathrm{V}(\mu)=\mu+\mu^{2} / k$, no algoritmo de ponderação local, a matriz de pesos $W$ será a mesma já apresentada na Seção 2.6 , ou seja, temos que $W=\operatorname{diag}\left(w_{1}, \ldots, w_{n}\right)$ com $w_{i}$ dado por (2.19).

3. Obtenha $\widehat{k}=\operatorname{argmax}_{k} l(k ; \widehat{\mu}, y)$ com o auxílio do algoritmo de NewtonRaphson, já descrito em (2.20).

4. Repita os passos 2 e 3 até convergência.

A implementação computacional desse algoritmo, passo a passo, no software S-Plus, foi baseada no comando glm.nb da biblioteca MASS (Venables e Ripley, 1997) e na função interna gam.nbut do pacote mgcv do software $R$ (Wood e Lonergan, 2002). Algumas funções da biblioteca MASS (Venables e Ripley, 1997) foram utilizadas para facilitar o processo de estimação do modelo aditivo binomial negativo: 
- Igamma (x): calcula $\log (\Gamma(x))$.

- digamma (x): calcula $\psi(x)=\Gamma^{\prime}(x) / \Gamma(x)$.

- trigamma $(\mathrm{x})$ : calcula $\psi^{\prime}(x)$.

-theta.mI (y, $\mu$, limit, trace): calcula a estimativa de máxima verossimilhança do parâmetro $k$, em $l(k ; \mu, y)$, com o critério de convergência dado pelo parâmetro da função limit e a impressão de cada passo do processo iterativo controlada pelo parâmetro da função trace. Essa função também calcula a estimativa do erro padrão de $\widehat{k}$, dado pela raiz do inverso da informação observada, ou seja, $\sqrt{\left(-\partial^{2} l(k ; \mu, y) / \partial k^{2}\right)^{-1}}$, avaliada nas estimativas dos parâmetros.

-negative.binomial(k): definição da distribuição binomial negativa com parâmetro conhecido $k$ para o ajuste de modelos de regressão. Apresenta também outros parâmetros, como por exemplo a especificação da função de ligação (link).

Com todas essas ferramentas, a implementação do algoritmo para o ajuste do modelo aditivo binomial negativo foi feita como uma função do S-Plus denominada gam.nb. Os comandos para construção dessa função estão apresentados no Apêndice A, e seus parâmetros são:

- G: especificação do modelo;

- link: função de ligação, que pode ser a função logarítmica ("log"') identidade ("identity") ou raiz quadrada ("sqrt" );

- control: parâmetro para critério de convergência; recomenda-se o uso do critério padrão do S-Plus, dado por gam. control();

- data: nome do banco de dados que será analisado.

Essa função apresenta os principais resultados passo a passo e, quando atinge a convergência, apresenta todos os resultados finais e a saída padrão do comando gam do S-Plus.

Exemplo 3.1 (continuação): Uma vez definida a função gam.nb, vamos ilustrar sua aplicação considerando o o conjunto de dados simulados apresentado na Figura 3.1 e o ajuste do modelo proposto em (3.25), mas supondo aqui que a variável resposta apresenta distribuição binomial negativa. Para tal, devemos utilizar o comando gam. $n b(y \sim 10(x, \operatorname{span}=0.30, d=2), 1$ ink $=$ "log", control $=$ gam. $\operatorname{control}()$, data $=$ dados $)$, que apresenta os resultados da forma apresentada a seguir. A Figura 3.12 ilustra os valores ajustados por esse modelo. 


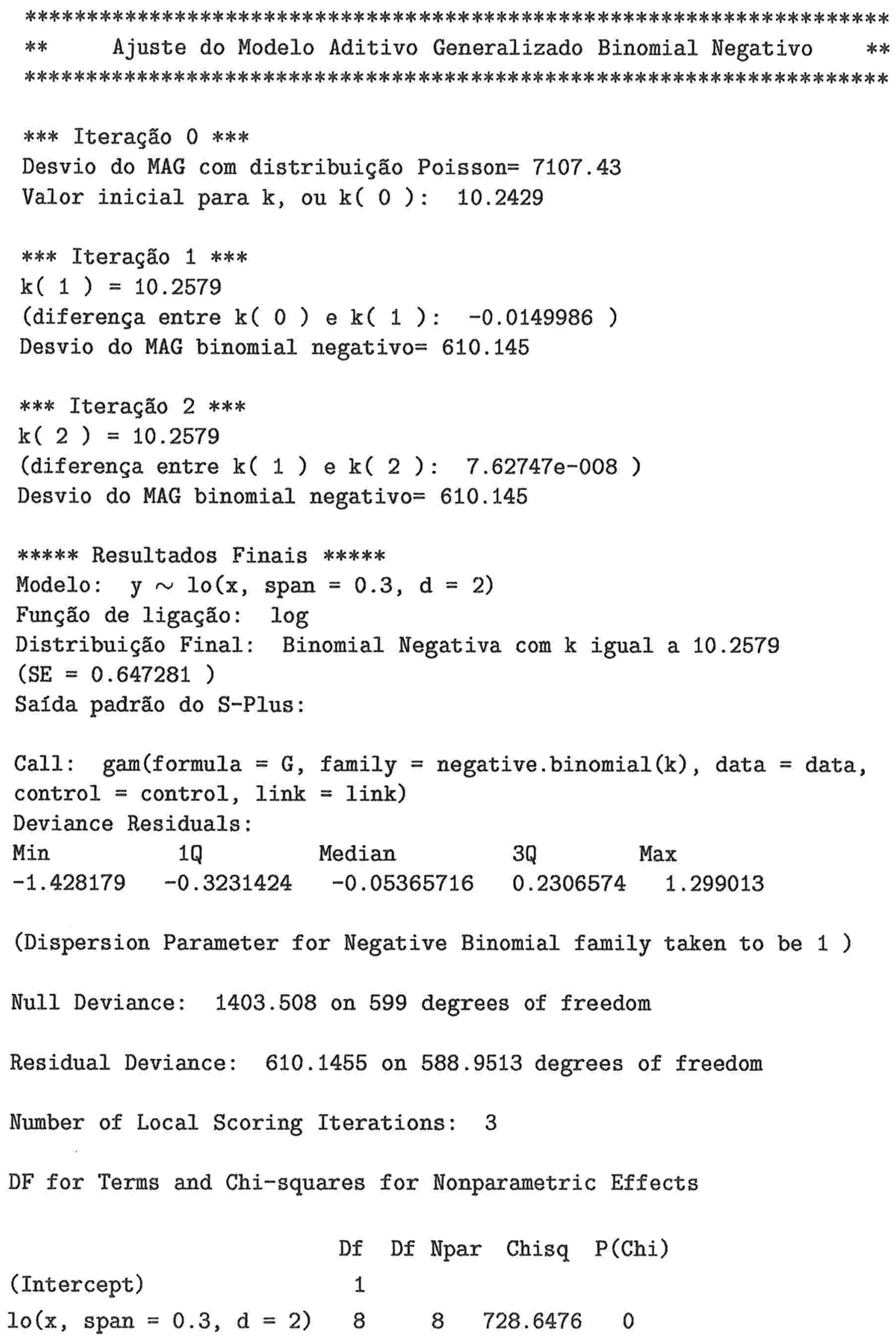




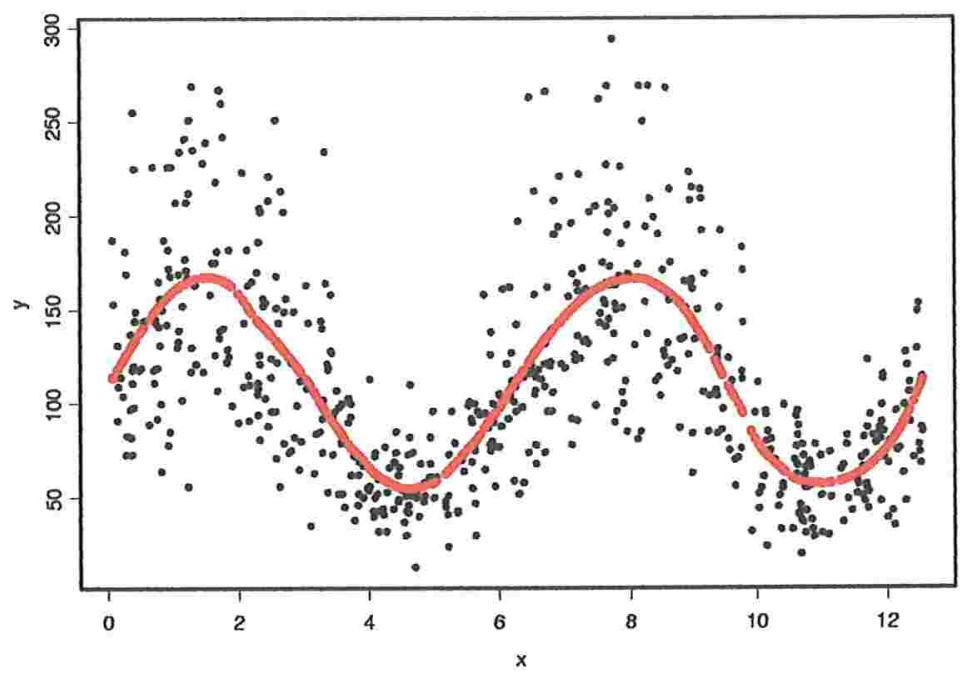

Figura 3.12: Valores ajustados pelo MAG binomial negativo.

A idéia para o ajuste de um modelo aditivo binomial negativo é muito similar à apresentada para os modelos lineares com distribuição binomial negativa na Seção 2.6. No entanto, para esse último demonstramos que as estimativas dos parâmetros $\beta$ e $k$, ambas obtidas pelo método de máxima verossimilhança, são assintoticamente independentes. Aqui, assim como em Thurston, Wand e Wiencke (2000), vamos desprezar o efeito que a estimação de $k$ pode ter no erro padrão da média estimada pelo modelo aditivo binomial negativo. No entanto, alguns autores criticam essa conduta pelo fato da estimação dos MAG's (Seção 3.3) ser feita através de estimativas de esperanças condicionais, tendo como base o algoritmo de retroajuste, e não exclusivamente pelo método de máxima verossimilhança.

A análise inferencial dos modelos aditivos binomiais negativos é feita exatamente como descrito para os MAG's na Seção 3.4, uma vez que aqui consideramos o parâmetro $k=\widehat{k}$ conhecido. Por esse mesmo motivo, o estudo inferencial de $\widehat{k}$ nesse contexto não parece fazer sentido. Por outro lado, sabemos que essa estimativa é obtida pelo método de máxima verossimilhança, e assim temos que uma estimativa da variância de $\widehat{k}$ pode ser obtida pelo inverso da informação observada, ou seja, $\left(-\partial^{2} l(k ; \mu, y) / \partial k^{2}\right)^{-1}$ avaliada nas estimativas dos parâmetros, que inclusive é calculada no ajuste desse modelo pela função gam.nb, apresentada anteriormente. Vale lembrar que a função $\partial^{2} l(k ; \mu, y) / \partial k^{2}$ está apresentada em (2.16). Por analogia aos resultados apresentados nas Seções 2.6 e 3.4, a avaliação da contribuição de covariadas no modelo aditivo binomial negativo deve ser feita através da estatística $\mathrm{F}$, dada em (3.28), pois nesse contexto o parâmetro de dis- 
persão $k$ é considerado conhecido, mas na verdade é substituído por sua estimativa de máxima verossimilhança $\widehat{k}$. O modelo sob investigação, com $q<p$ covariadas, deve ser ajustado com o valor de $k$ obtido no ajuste do modelo irrestrito, com $p$ covariadas.

Logo, o teste $\mathrm{F}$ aproximado (Seção 3.4) é também mais apropriado para avaliar se uma covariada em especial apresenta maior contribuição no modelo de forma linear ou não paramétrica, e por analogia ao teste $\mathrm{F}$ para avaliar a contribuição de covariadas, temos que o modelo linear deve ser ajustado com a estimativa de $k$ obtida no ajuste do modelo aditivo.

Exemplo 3.1 (continuação): Pelo teste F, concluímos que existe associação significativa entre $x$ e $y(p<0,001)$. O teste $\mathrm{F}$ para comparação do modelo não paramétrico com o modelo de regressão linear binomial negativo apresenta estatística igual a 74,76 ( $p<0,001)$, indicando que a associação entre $x$ e $y$ é melhor representada por uma função não paramétrica, como esperado pela Figura 3.1. A função não paramétrica estimada e respectiva banda de confiança estão representadas na Figura 3.13. As linhas pontilhadas são uma aproximação para o intervalo de confiança da curva de alisamento ajustada, e as barras verticais no eixo das abscissas representam os valores de $x$ efetivamente observados na amostra.

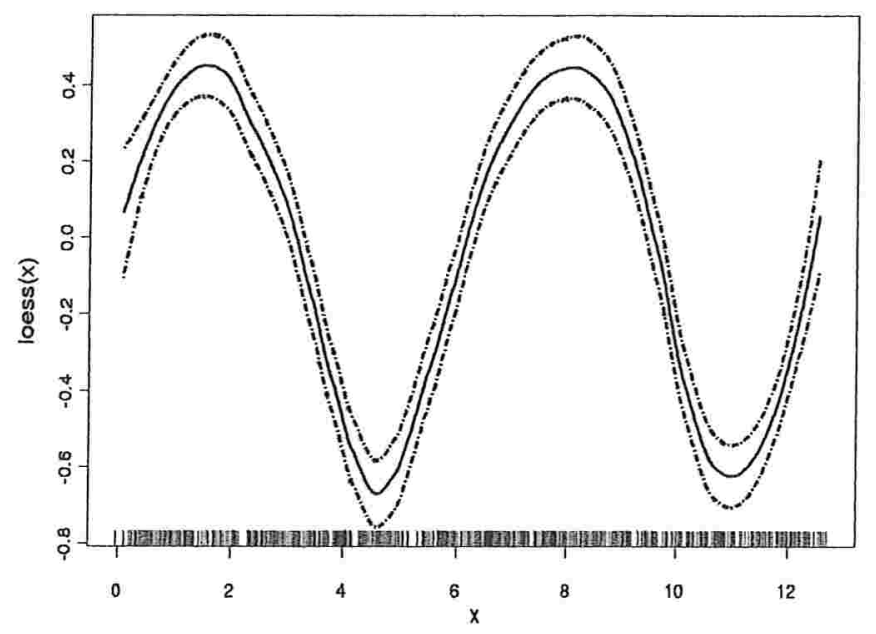

Figura 3.13: Gráfico da função loess estimada com $\lambda=30 \%$ e $d=2$ no modelo de regressão de binomial negativo. 
Na prática, a seleção dos parâmetros das funções alisadoras dos modelos binomiais negativos não é uma tarefa simples pelo fato do parâmetro de dispersão $k$ ser em geral desconhecido. Da mesma forma, a seleção desses modelos também se torna difícil. Para cada modelo ajustado temos uma estimativa $\widehat{k}$ diferente que irá influenciar, juntamente com $\widehat{\mu}$, o cálculo dos desvios dos modelos, e logo, as medidas $V C_{D}$ e $A I C$ apresentadas na Seção 3.5. Assim, não podemos concluir que os modelos com menores valores do desvio ou das medidas $V C_{D}$ e $A I C$ são mais adequados, uma vez que esse resultado pode ser devido ao valor de $\widehat{k}$, e não de $\widehat{\mu}$. Parece claro que para validar a proposta de comparação de modelos descrita na Seção 3.5 para os modelos aditivos binomais negativos, seria necessário fixar um valor para $k$, o que na prática seria outra questão difícil. Assim, podemos dizer que a avaliação gráfica dos dados sobrepostos às curvas de alisamento para diversos valores de $\lambda$ e a aplicação de técnicas de diagnóstico para verificar a qualidade do ajuste desses modelos são especialmente importantes na seleção e comparação de modelos aditivos binomiais negativos.

A análise de diagnóstico do modelo aditivo binomial negativo com $k=\widehat{k}$ considerado conhecido deve ser feita exatamente como descrito na Seção 3.6.

Exemplo 3.1 (continuação): Para ilustrar o procedimento de seleção dos parâmetros de alisamento de funções alisadoras no ajuste dos modelos aditivos binomiais negativos, consideramos o ajuste dos modelos aditivos $\mu_{i}=\exp \left(\beta_{0}+\right.$ loess $\left(x_{i}, \lambda, d\right)$ ) com distribuição binomial negativa e calculamos a função de Akaike $(A I C)$, a validação cruzada $\left(V C_{D}\right)$ e a soma dos quadrados das autocorrelações dos resíduos com defasagem de 1 a $30\left(\sum_{i=1}^{30} F A C_{i}^{2}\right)$ para um pequeno conjunto de valores de $\lambda \operatorname{com} d=1$ e $d=2$. Essas medidas estão apresentadas na Tabela 3.1, juntamente com a estimativa $\widehat{k}$ de cada modelo. A avaliação gráfica desses parâmetros está apresentada na Figura 3.14, onde temos as estimativas do preditor de cada modelo sobrepostas aos dados, ou seja, gráficos do $\log (y)$ contra $x$ e $\widehat{\eta}$ contra $x$. Os gráficos de probalidade meio normal com envelope simulado do valor absoluto dos resíduos componentes do desvio dos modelos avaliados estão apresentados na Figura 3.15.

Avaliando esses resultados, podemos observar que, entre os modelos estudados, o modelo com parâmetros $\lambda=60 \%$ e $d=1$ é o que apresenta a menor estimativa do parâmetro de dispersão $k$ e apresenta os menores valores da função $A I C$, da soma dos quadrados das autocorrelações dos resíduos com defasagem de 1 a 30 e da função $V C_{D}$, mas pela avaliação gráfica da Figura 3.14 observamos que esse modelo apresenta a curva estimada muito lisa, não representando a relação de $x$ e $y$ de forma adequada. As estimativas do parâmetro $k$ apresentaram 
pequena amplitude entre os parâmetros estudados (de 6,22 a 10,56) e nesse caso o gráfico de probalidade meio normal com envelope simulado foi pouco informativo, uma vez que indica boa qualidade de ajuste para todos os modelos estudados (ver Figura 3.15). Pela análise gráfica das curvas estimadas na Figura 3.14, observamos que o modelo com parâmetros $\lambda=30 \%$ e $d=2$ parece reproduzir muito bem a tendência de $y$ em relação a $x$. Por outro lado, em relação aos demais, esse modelo apresenta valores intermediários para as medidas da Tabela 3.2. Com isso, confirmamos que nesse procedimento essas medidas não são informativas, e portanto seus cálculos não são recomendados, também pelo alto custo computacional principalmente da $V C_{D}$. Assim fica evidente a necessidade e importância da avaliação gráfica das curvas estimadas sobrepostas aos dados na escolha desses modelos.

Na Figura 3.16 temos os gráficos dos resíduos de Pearson contra os valores ajustados e da função de autocorrelação (FAC) dos resíduos de Pearson para o modelo aditivo binomial negativo com $\lambda=30 \%$ e $d=2$, que não indicam nenhuma evidência de falta de ajuste. Concluindo, de uma forma geral, podemos dizer que o modelo com parâmetros $\lambda=30 \%$ e $d=2$ parece adequado para a análise desses dados.

Tabela 3.2: Valores da função de Akaike $(A I C)$, da validação cruzada $\left(V C_{D}\right)$ e da soma dos quadrados das autocorrelações dos resíduos com defasagem de 1 a $30\left(\sum_{i=1}^{30} F A C_{i}^{2}\right)$ para um pequeno conjunto de valores de $\lambda \operatorname{com} d=1$ e $d=2$ no ajuste de modelos aditivos com distribuição binomial negativa, e respectivas estimativas do parâmetro de dispersão $k$.

\begin{tabular}{cccccc}
\hline$\lambda$ & $d$ & $A I C$ & $\sum_{i=1}^{30} F A C_{i}^{2}$ & $V C_{D}$ & $\widehat{k}$ \\
\hline \hline $15 \%$ & 1 & 1,060 & 1,042 & 1,061 & 10,286 \\
$30 \%$ & 1 & 1,043 & 1,043 & 1,043 & 9,080 \\
$45 \%$ & 1 & 1,040 & 1,043 & 1,042 & 7,200 \\
$60 \%$ & 1 & 1,040 & 1,042 & 1,040 & 6,220 \\
$15 \%$ & 2 & 1,088 & 1,040 & 1,092 & 10,557 \\
$30 \%$ & 2 & 1,054 & 1,043 & 1,055 & 10,258 \\
$45 \%$ & 2 & 1,043 & 1,043 & 1,045 & 9,752 \\
$60 \%$ & 2 & 1,042 & 1,046 & 1,043 & 8,038 \\
\hline
\end{tabular}



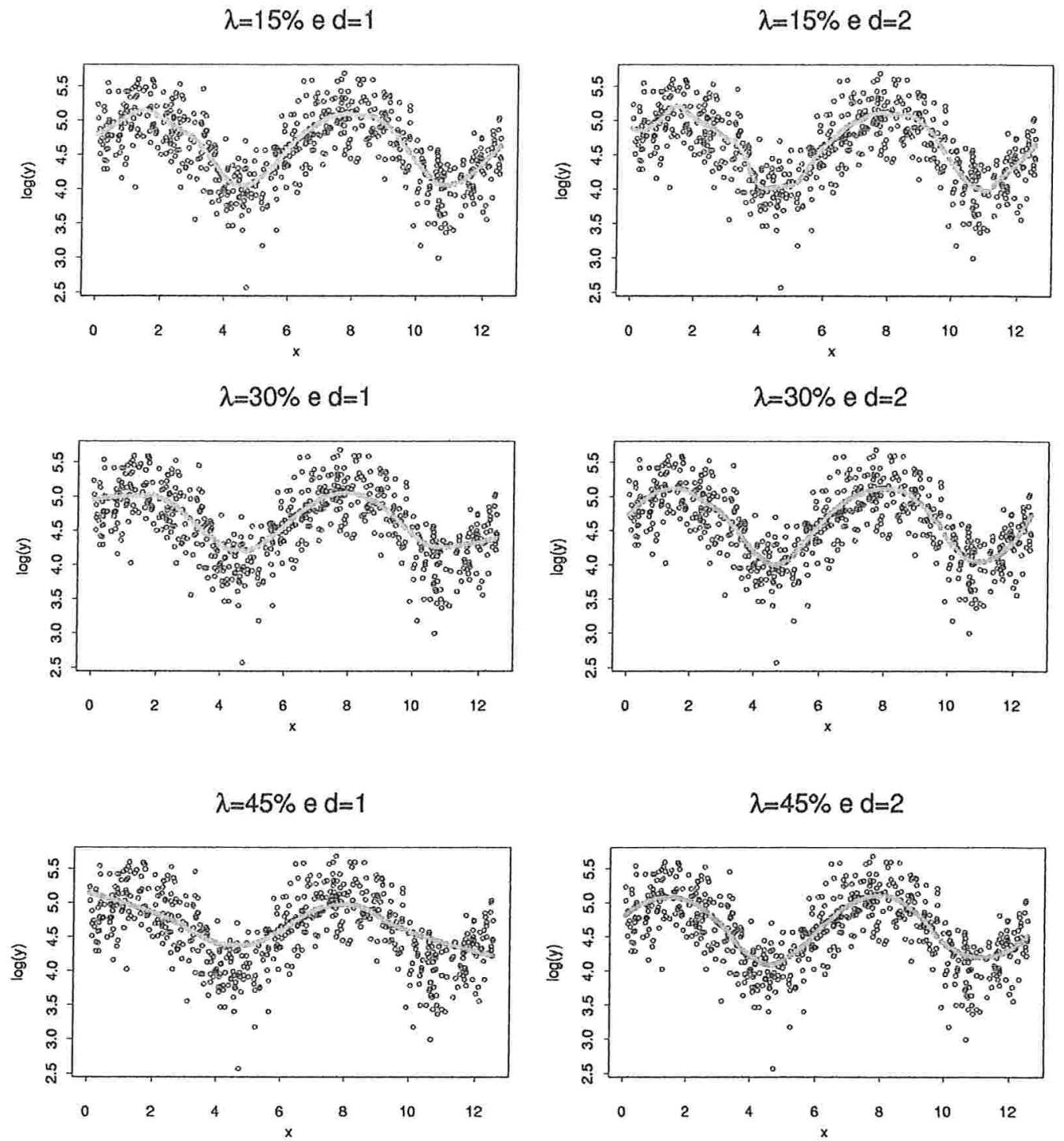

$\lambda=60 \%$ e $d=1$

$\lambda=60 \%$ e $d=2$
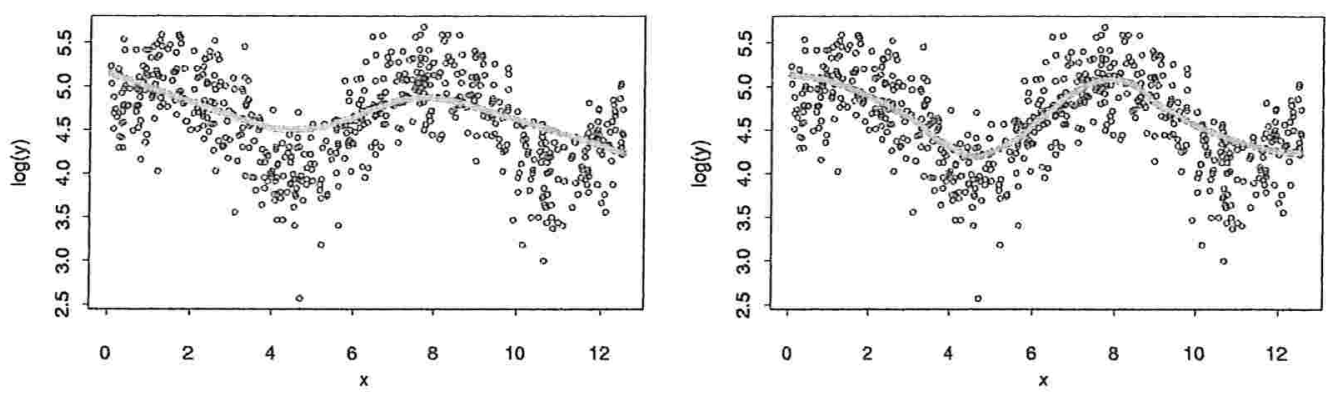

Figura 3.14: Estimativas do preditor do modelo aditivo binomial negativo com curvas alisadas do tipo loess com diferentes valores de $\lambda$ e $d$, sobrepostas aos dados. 

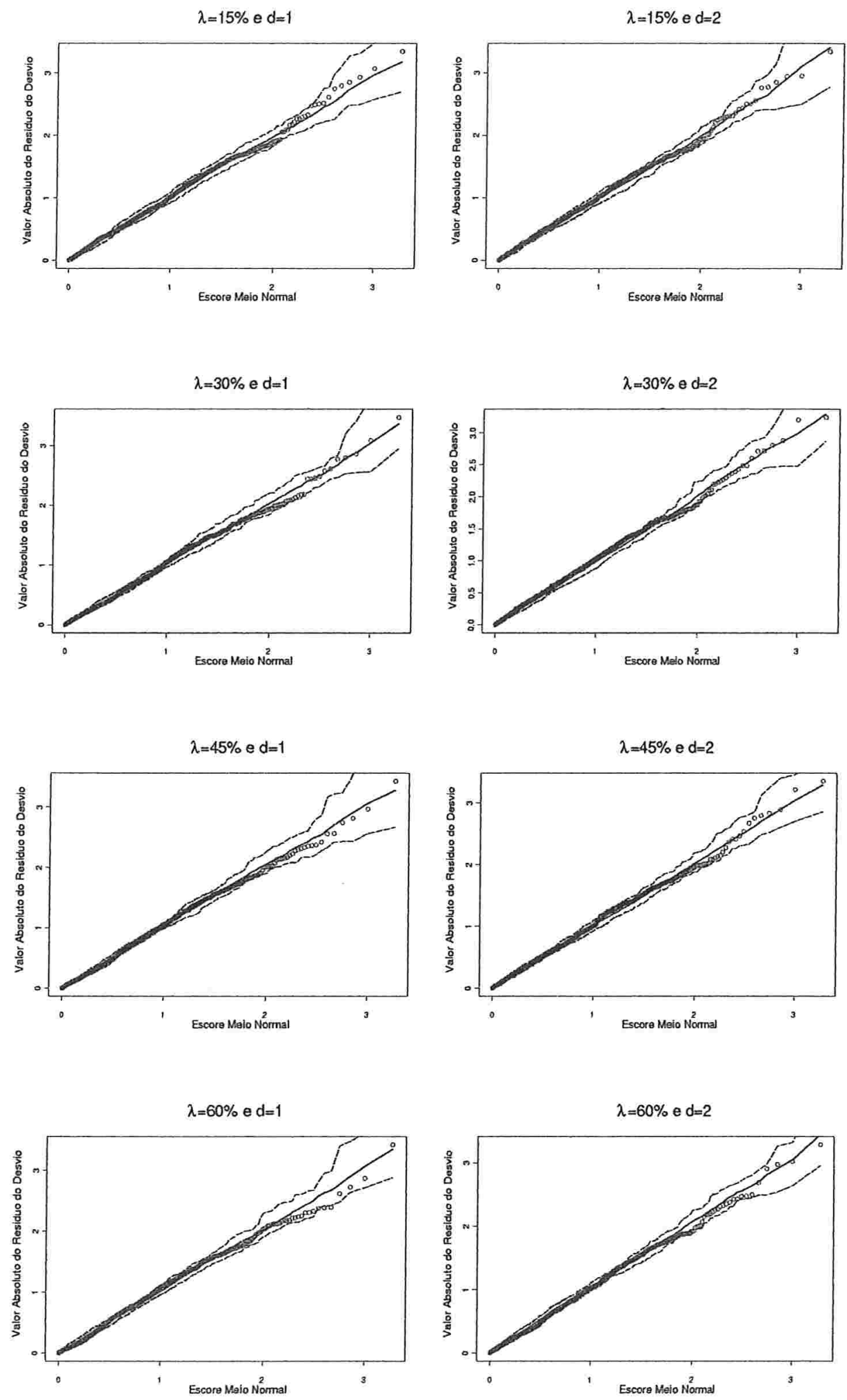

Figura 3.15: Gráficos de probabilidade meio normal com envelpe simulado do valor absoluto dos resíduos do desvio dos modelos aditivos binomiais negativos com diferentes valores de $\lambda$ e $d$. 
(a)

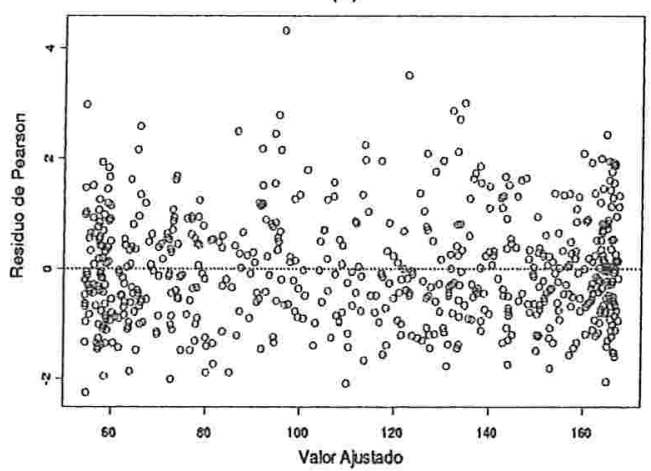

(b)

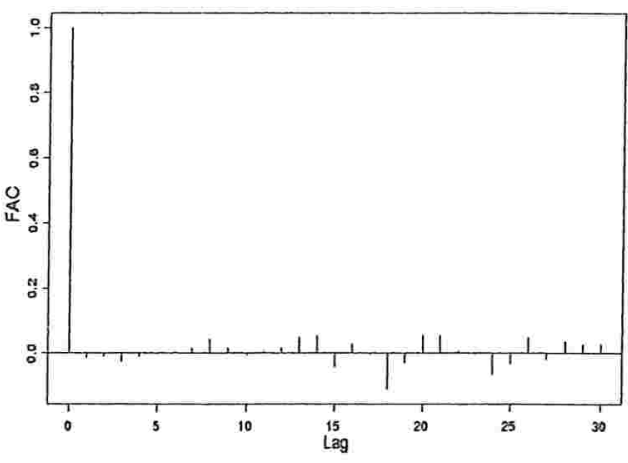

Figura 3.16: Gráfico dos resíduos de Pearson contra os valores ajustados (a) e gráfico da função de autocorrelação (FAC) dos resíduos de Pearson (b) para o modelo aditivo binomial negativo.

Uma vez que destacamos a importância da análise de diagnóstico na escolha de modelos aditivos binomiais negativos e nesse exemplo esta foi pouca informativa pela pequena amplitude de variação da estimativa $k$, propomos o ajuste do modelo aditivo binomial negativo $\mu_{i}=\exp \left(\beta_{0}+\operatorname{loess}\left(x_{i}, \lambda=30 \%, d=2\right)\right)$ para diferentes valores de $k$, sendo esses $3,10,30$ e 60 , lembrando que seu "verdadeiro valor", ou seja, pelo qual os dados foram gerados, é igual a 10 .

Os valores da função de Akaike $(A I C)$, da validação cruzada $\left(V C_{D}\right)$ e da soma dos quadrados das autocorrelações dos resíduos com defasagem de 1 a 30 $\left(\sum_{i=1}^{30} F A C_{i}^{2}\right)$ desses modelos estão apresentadas na Tabela 3.3, e seus gráficos de probabilidade meio normal com envelope simulado do valor absoluto dos resíduos do desvio estão na Figura 3.17. Avaliando esses resultados, observamo nitidamente que as medidas da Tabela 3.3 dependem muito do valor de $k$, onde temos que o menor valor de $k$ considerado é que apresenta os menores valores da tabela. Por outro lado, quando avaliamos os gráficos de probabilidade meio normal com envelope simulado da Figura 3.17, podemos afirmar que o modelo aditivo bimomial negativo com parâmetro de dispersão $k=10$, como esperado, é o que apresenta o melhor ajuste.

Com isso, podemos concluir que a realização da análise de diagnóstico, juntamente com a avaliação gráfica dos dados em estudo, são fundamentais na seleção dos parâmetros de funções alisadoras, e logo, na seleção de modelos aditivos binomiais negativos, uma vez que as medidas usuais para seleção de modelos propostas na literatura não têm aplicabilidade nesse contexto. 
Tabela 3.3: Valores da função de Akaike $(A I C)$, da validação cruzada $\left(V C_{D}\right)$ e da soma dos quadrados das autocorrelações dos resíduos com defasagem de 1 a $30\left(\sum_{i=1}^{30} F A C_{i}^{2}\right)$ para $\lambda=30 \%$ e $d=2$ no ajuste de modelos aditivos com distribuição binomial negativa e diversos valores do parâmetro de dispersão $k$.

\begin{tabular}{cccc}
\hline$k$ & $A I C$ & $\sum_{i=1}^{30} F A C_{i}^{2}$ & $V C_{D}$ \\
\hline \hline 3 & 0,358 & 1,042 & 0,333 \\
10 & 1,031 & 1,043 & 1,031 \\
30 & 2,535 & 1,044 & 2,594 \\
60 & 4,096 & 1,044 & 4,216 \\
\hline
\end{tabular}

$k=3$

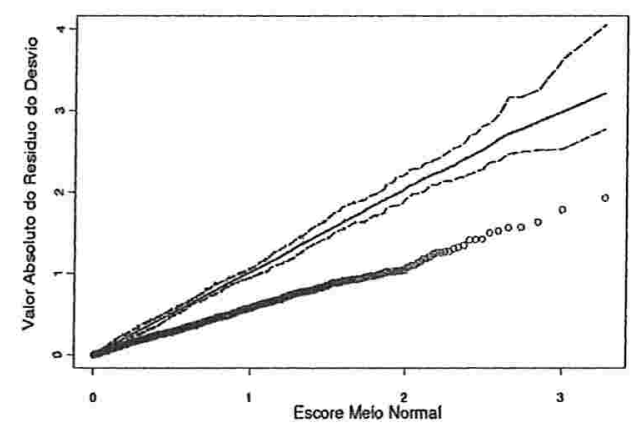

$k=30$

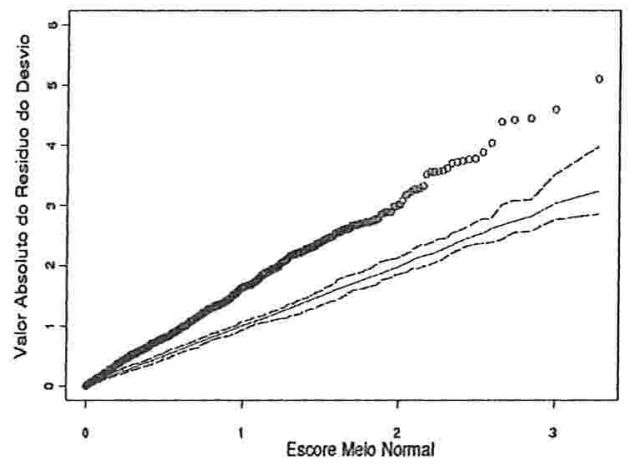

$k=10$

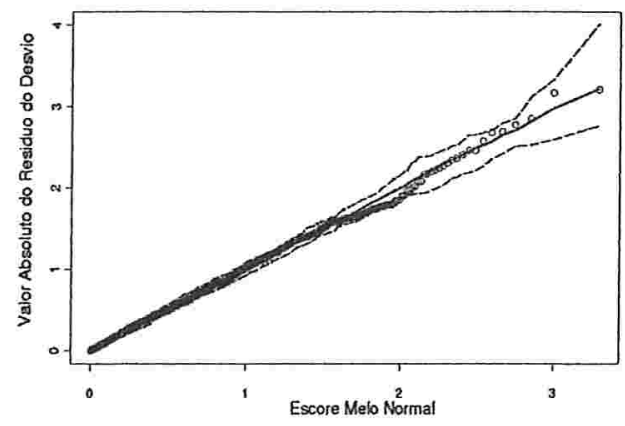

$k=60$

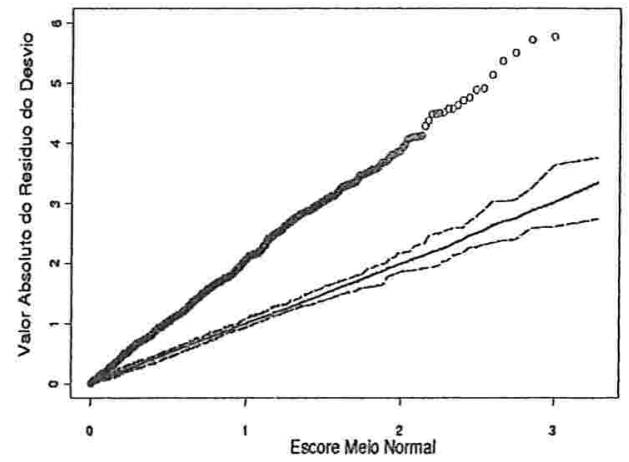

Figura 3.17: Gráficos de probabilidade meio normal com envelpe simulado do valor absoluto dos resíduos componentes do desvio para diversos valores de $k$, $\operatorname{com} \lambda=30 \%$ e $d=2$. 


\subsection{Risco Relativo}

O risco relativo, já definido na Seção 2.7 , é uma medida de grande interesse não só no contexto dos MLG's como também no dos MAG's. Da mesma forma que nos MLG's, o risco relativo expressa o impacto de cada uma das covariadas na variável resposta, e é calculado considerando a função de ligação logarítmica $g\left(\mu_{i}\right)=\log \mu_{i}$. Para o estudo do risco relativo vamos considerar o seguinte MAG

$$
\mu_{i}=\exp \left[\sum_{l=1}^{p_{1}} \beta_{l} x_{i l}+\sum_{l=p_{1}+1}^{p} f_{l}\left(x_{i l}\right)\right] .
$$

Vamos primeiramente mostrar que a definição do risco relativo para qualquer uma das $p_{1}$ primeiras covariadas do MAG (3.32), ou seja, as covariadas que estão relacionadas a um parâmetro do modelo, recai na expressão (2.24). Sejam $x_{h}=\left(1, \ldots, x_{h j}, \ldots, x_{h p_{1}}, x_{h p_{1}+1} \ldots, x_{h p}\right)^{\top}$ um vetor de valores das covariadas e $x_{h^{*}}=\left(1, \ldots, x_{h j}+c, \ldots, x_{h p_{1}}, x_{h p_{1}+1}, \ldots, x_{h p}\right)^{\top}$. As médias $\mu_{h}$ e $\mu_{h^{*}}$ da variável resposta dados $x_{h}$ e $x_{h^{*}}$, respectivamente, são dadas pelo modelo (3.32). Note-se que o risco relativo é dado por

$R R_{j}=\frac{\mu_{h^{*}}}{\mu_{h}}=\frac{\exp \left[\sum_{l=1, l \neq j}^{p_{1}} \beta_{l} x_{h l}+\beta_{j}\left(x_{h j}+c\right)+\sum_{l=p_{1}+1}^{p} f_{l}\left(x_{h l}\right)\right]}{\exp \left[\sum_{l=1}^{p_{1}} \beta_{l} x_{h l}+\sum_{l=p_{1}+1}^{p} f_{l}\left(x_{h l}\right)\right]}=\exp \left(c \beta_{j}\right)$

e, portanto, $c \beta_{j}$ é o logaritmo desse risco quando o valor da $j$-ésima covariada é acrescido de $c$ unidades e as outras covariadas permanecem inalteradas. Assim, uma estimativa desse risco é dada por

$$
\widehat{R R}_{j}=\frac{\widehat{\mu}_{h^{*}}}{\widehat{\mu}_{h}}=\exp \left(c \widehat{\beta}_{j}\right)
$$

Logo, temos que $\exp \left(\widehat{\beta}_{j}\right)$ é uma estimativa do risco relativo ao aumento de uma unidade no valor da $j$-ésima covariada, mantendo as outras covariadas constantes.

Pelos resultados apresentados na Seção 3.4 podemos construir um intervalo de confiança assintótico aproximado para o risco relativo ao aumento de $c$ unidades na $j$-ésima covariada quando as outras covariadas permanecem inalteradas, que será dado por

$$
\exp \left[c\left(\widehat{\beta}_{j} \mp z_{\alpha / 2} \sqrt{\widehat{v}_{j j}}\right)\right]
$$

onde $z_{\alpha}$ é o quantil de ordem $1-\alpha$ de distribuição normal padrão e $\widehat{v}_{j j}$ é o elemento $(j, j)$ da matriz de variância e covariância aproximada de $\widehat{\beta}$, dada em (3.30). Sem perda de generalidade, a curva de risco relativo em função de $c$, proposta por Singer et al.(2002), também pode ser construída como descrito na Seção 2.7. 
Agora vamos vamos definir o risco relativo para qualquer uma das $p-p_{1}$ últimas covariadas do MAG (3.32), ou seja, as covariadas que estão avaliadas de forma não paramétrica no modelo através de uma função alisadora. Sejam $x_{h}=\left(1, \ldots, x_{h p_{1}}, x_{h p_{1}+1}, \ldots, x_{h j}, \ldots, x_{h p}\right)^{\top}$ um vetor de valores das covariadas e $x_{h^{*}}=\left(1, \ldots, x_{h p_{1}}, x_{h p_{1}+1}, \ldots, x_{h j}+c, \ldots, x_{h p}\right)^{\top}$. As médias $\mu_{h}$ e $\mu_{h^{*}}$ da variável resposta dados $x_{h}$ e $x_{h^{*}}$, respectivamente, são dadas pelo modelo (3.32). Nesse caso, note-se que o risco relativo é dado por

$$
\begin{aligned}
R R_{j} & =\frac{\mu_{h^{*}}}{\mu_{h}}=\frac{\exp \left[\sum_{l=1}^{p_{1}} \beta_{l} x_{h l}+\sum_{l=p_{1}+1, l \neq j}^{p} f_{l}\left(x_{h l}\right)+f_{j}\left(x_{h j}+c\right)\right]}{\exp \left[\sum_{l=1}^{p_{1}} \beta_{l} x_{h l}+\sum_{l=p_{1}+1}^{p} f_{l}\left(x_{h l}\right)\right]} \\
& =\exp \left[d\left(x_{h j}, c\right)\right],
\end{aligned}
$$

onde $d\left(x_{h j}, c\right)=f_{j}\left(x_{h j}+c\right)-f_{j}\left(x_{h j}\right)$, ou seja, nesse caso o risco relativo depende não só de $c$ como também do valor da covariada de referência $x_{h j}$. Aqui, $f_{j}\left(x_{h j}+c\right)-f_{j}\left(x_{h j}\right)$ é o logaritmo desse risco quando o valor de referência $x_{h j}$ da $j$-ésima covariada é acrescido de $c$ unidades e as outras covariadas permanecem inalteradas. Assim, uma estimativa desse risco é dada por

$$
\widehat{R R}\left(x_{h j}, c\right)=\frac{\widehat{\mu}_{h^{*}}}{\widehat{\mu}_{h}}=\exp \left[\widehat{d}\left(x_{h j}, c\right)\right]
$$

onde $\widehat{d}\left(x_{h j}, c\right)=\widehat{f}_{j}\left(x_{h j}+c\right)-\widehat{f}_{j}\left(x_{h j}\right)$. Recomenda-se que o RR seja calculado para valores de $x_{h j}+c$ e $x_{h j}$ observados na amostra, pois no caso contrário seria necessário obter $\widehat{f}_{j}\left(x_{h j}+c\right)$ e $\widehat{f}_{j}\left(x_{h j}\right)$ através de processos de interpolação aplicados à função $\widehat{f_{j}}$.

Para construir um intervalo de confiança assintótico para o risco relativo ao aumento de $c$ unidades no valor de referência $x_{h j}$ da $j$-ésima covariada, quando as outras covariadas permanecem inalteradas, é necessário obter uma estimativa do erro padrão do estimador dado em (3.33). Lima (2001, Seçăo 4.4) apresenta como sugestão para obter o erro padrão de $\widehat{R R}\left(x_{h j}, c\right)$, a expansão de $\widehat{d}\left(x_{h j}, c\right)$ em série de Taylor em torno de $d\left(x_{h j}, c\right)=f_{j}\left(x_{h j}+c\right)-f_{j}\left(x_{h j}\right)$ até primeira ordem, obtendo

$$
\widehat{R R}\left(x_{h j}, c\right)=\exp \left[\widehat{d}\left(x_{h j}, c\right)\right] \approx \exp \left[d\left(x_{h j}, c\right)\right]+\exp \left[d\left(x_{h j}, c\right)\right]\left[\widehat{d}\left(x_{h j}, c\right)-d\left(x_{h j}, c\right)\right]
$$

Então

$$
\begin{aligned}
\operatorname{Var}\left\{\widehat{R P}\left(x_{h j}, c\right)\right\}= & \operatorname{Var}\left\{\exp \left[\widehat{d}\left(x_{h j}, c\right)\right]\right\} \approx \exp ^{2}\left[d\left(x_{h j}, c\right)\right] \operatorname{Varr}\left\{\widehat{d}_{\left.\left(x_{j k}, c\right)\right\}}\right. \\
= & \exp ^{2}\left[d\left(x_{h j}, c\right)\right]\left\{\operatorname{Var}\left\{\widehat{f}_{j}\left(x_{h j}+c\right)\right\}+\operatorname{Var}\left\{\widehat{f}_{j}\left(x_{h j}\right)\right\}\right. \\
& \left.-2 \operatorname{Cov}\left\{\widehat{f}_{j}\left(x_{h j}+c\right), \widehat{f}_{j}\left(x_{h j}\right)\right\}\right\}
\end{aligned}
$$


Lima (2001) conclui que um estimador da $\operatorname{Var}\left\{\widehat{R R}\left(x_{h j}, c\right)\right\}$ pode ser obtido pela substituição de $d\left(x_{h j}, c\right)$ por $\widehat{d}\left(x_{h j}, c\right)$ na sua expressão, assumindo a consistência de $\widehat{f}_{j}$. Logo, temos

$$
\begin{aligned}
\left.\widehat{\operatorname{Var}}\left\{\widehat{R R}\left(x_{h j}, c\right)\right)\right\}= & \exp ^{2}\left[\widehat{d}\left(x_{h j}, c\right)\right]\left\{\operatorname{Var}\left\{\widehat{f}_{j}\left(x_{h j}+c\right)\right\}\right. \\
& \left.+\operatorname{Var}\left\{\widehat{f_{j}}\left(x_{h j}\right)\right\}-2 \operatorname{Cov}\left\{\widehat{f}_{j}\left(x_{h j}+c\right), \widehat{f}_{j}\left(x_{h j}\right)\right\}\right\}
\end{aligned}
$$

As estimativas das variâncias e covariância envolvidas nesta expressão são obtidas a partir de (3.29), como descrito na Seção 3.3.

Finalmente, temos que um intervalo de confiança assintótico para o risco relativo ao aumento de $c$ unidades no valor de referência $x_{h j}$ da $j$-ésima covariada, considerando constantes as demais covariadas, é dado por

$$
\widehat{R R}\left(x_{h j}, c\right) \mp z_{\alpha / 2} \sqrt{\widehat{\operatorname{Var}}\left\{\widehat{R R}\left(x_{h j}, c\right)\right\}},
$$

onde $z_{\alpha}$ é o quantil de ordem $1-\alpha$ de distribuição normal padrão, e $\widehat{R R}\left(x_{h j}, c\right)$ e $\widehat{\operatorname{Var}}\left\{\widehat{R R}\left(x_{h j}, c\right)\right\}$ são dados por (3.33) e (3.34), respectivamente.

Assim, podemos construir uma curva pontual do risco relativo com bandas de confiança em função de $c$ para um determinado valor de referência da $j$-ésima covariada $x_{h j}$. Essa curva é dita pontual porque é construída com base nos valores de $c$ da amostra para evitar o processo de interpolação, como já comentado anteriormente. Lima (2001) sugere a construção dessa curva considerando como valor de referência o valor mínimo da $j$-ésima covariada observado na amostra. Dessa forma a curva de risco relativo pontual apresenta o número máximo de pontos $(n-1)$. Uma outra sugestão seria considerar o primeiro quartil da $j$ ésima covariada como o valor de referência, e construir essa curva para valores de $c$ tais que $x_{h j}+c$ fosse no máximo o terceiro quartil da $j$-ésima covariada. Essa idéia seria uma tentativa de avaliar o risco relativo sem considerar diretamente os valores mais discrepantes da $j$-ésima covariada observados na amostra. No próximo capítulo apresentaremos uma comparação dessas curvas. 


\section{Capítulo 4}

\section{Associação entre poluição atmosférica e saúde em São Paulo}

A cidade de São Paulo, a maior da América do Sul, tem sido objeto de estudos epidemiológicos para avaliar a associação entre poluição atmosférica e saúde humana; ver, por exemplo, Saldiva et al. $(1994,1995)$, Braga et al. $(1999,2001)$ e Conceição et al. (2001).

São Paulo é um excelente local para avaliar os efeitos da poluição atmosférica na saúde. É o maior centro urbano industrializado da América Latina, com uma população de aproximadamente 10 milhões de habitantes. A frota de veículos automotores da Região Metropolitana de São Paulo é de cerca de 6 milhões de veículos, os quais constituem a principal fonte de poluição do ar, seguidos pelos processos industriais (CETESB, 2001). Devido às suas características geográficas, São Paulo apresenta freqüentes inversões térmicas que dificultam a dispersão de poluentes resultando em um aumento substancial da poluição atmosférica. Além disso, a agência de controle da poluição atmosférica de São Paulo (CETESB) apresenta o registro diário da concentração de vários poluentes através de 13 estações de monitoramento da qualidade do ar espalhadas pela cidade, facilitando a realização de estudos temporais relativos à poluição atmosférica. A grande massa populacional permite sua divisão em menores grupos de especial interesse, já que a literatura aponta que crianças, idosos e pessoas que apresentam doenças crônicas são mais suscetíveis à poluição do ar.

Esse estudo epidemiológico é do tipo ecológico, no qual a unidade de observação é a população e não o indivíduo. Além desse tipo de estudo ser simples e barato por aproveitar dados coletados rotineiramente por órgãos governamentais, ele tem a vantagem de que a população age como seu próprio controle evitando fatores de confundimento que diferentes populações poderiam gerar.

Nesse estudo nosso foco é investigar a àssociação entre o número de atendimentos pediátricos de emergência por causas respiratórias e os níveis de poluição atmosférica na cidade de São Paulo. Trata-se de uma nova análise de parte dos dados estudados 
"Os resultados nunca vêm antes de nos arriscarmos."

(Wes Beavis) 
por Lin et al. (1999), os quais utilizam modelos de regressão de Poisson para avaliar tal associação. Primeiramente, mostramos que o modelo de regressão log-linear binomial negativo é mais adequado à modelagem do conjunto de dados considerado do que o modelo de regressão log-linear de Poisson. Em seguida, avaliamos essa associação através de um modelo aditivo binomial negativo, e comparamos alguns resultados dos modelos estudados. Esta análise está relacionado com a de Ferrari e outros (2002) que utilizam modelos de superdispersão para análise de associação entre a concentração de dióxido de enxofre e o número de atendimentos por causas respiratórias em um pronto socorro infantil em Vitória, Espírito Santo.

Registros diários do número de atendimentos pediátricos de emergência por causas respiratórias foram originalmente obtidos no Instituto da Criança da Universidade de São Paulo (ICUSP) no período entre maio de 1991 e abril de 1993. No nosso estudo esses eventos representam o indicador de saúde da população.

$\mathrm{O}$ indicador de poluição utilizado foi a concentração média diária de material particulado $\left(\mathrm{MP}_{10}, \mathrm{em} \mu \mathrm{g} / \mathrm{m}^{3}\right)$ e procuramos controlar o efeito de variáveis de confundimento. $\mathrm{O} \mathrm{MP}_{10}$ é uma das principais classes de poluentes na atmosfera, de grande importância como parâmetro de qualidade do ar, e suas características são: partículas de material sólido ou líquido que, devido a seu pequeno tamanho $(<10 \mu m)$, ficam suspensas no ar na forma de poeira, neblina, aerossol, fumaça, fuligem, entre outras. Para descrever as características do meio ambiente foram incluídas: a temperatura $\left({ }^{\circ} \mathrm{C}\right)$, representada pela média diária das temperaturas mínimas observadas em diferentes estações meteorológicas, e a umidade relativa do ar (\%), representada pela média diária dos valores registrados ao meio-dia. Para representar fatores sazonais incluímos variáveis indicadoras para cada mês do período em estudo e dia da semana. O número total de atendimentos pediátricos de emergência por causas não respiratórias foi também considerado, objetivando controlar fatores externos como, por exemplo, greve no hospital.

A Tabela 4.1 mostra medidas resumo anuais de algumas variáveis em estudo. A Figura 4.1 apresenta as séries de dados do número de visitas pediátricas de emergência por causas respiratórias (RESP) e da concentração de $\mathrm{MP}_{10}$. Note-se que há períodos sem registros da variável resposta e alguns dias onde a concentração média de $\mathrm{MP}_{10}$ ultrapassou o valor de referência internacional de qualidade do ar $\left(150 \mu \mathrm{g} / \mathrm{m}^{3}\right)$.

A estratégia de construção do modelo para a análise estatística envolveu a determinação de um modelo básico no qual efeitos devidos a variáveis climáticas, tendências sazonais e outros fatores de confundimento foram removidos. A idéia é explicar ao máximo a variabilidade no número de atendimentos pediátricos de emergência através do controle de variáveis de confundimento, definindo um modelo básico antes de adicionar a concentração do poluente no modelo. Estratégias similares para a construção do modelo de análise são apresentadas por Conceição et al. (2001), Schwartz e outros (1996) e Singer et al. (2002). Toda a análise estatística que se segue foi realizada com o auxílio do software S-Plus 4.5. 
Tabela 4.1. Número de observações $(n)$ e médias (desvios padrão) anuais do número de visitas pediátricas de emergência por causas respiratórias (RESP) e não-respiratórias (NRESP), temperatura ( $\mathrm{T}$ ), umidade (U) e concentração de $\mathrm{MP}_{10}$.

\begin{tabular}{cccccc}
\hline Ano & RESP & NRESP & $\mathrm{T}\left({ }^{\circ} \mathrm{C}\right)$ & $\mathrm{U}(\%)$ & $\mathrm{MP}_{10}\left(\mu \mathrm{g} / \mathrm{m}^{3}\right)$ \\
\hline \hline 1991 & 56,95 & 119,37 & 14,17 & 65,23 & 76,42 \\
$(n=226)$ & $(19,59)$ & $(44,90)$ & $(2,86)$ & $(13,45)$ & $(34,10)$ \\
\hline 1992 & 54,02 & 120,97 & 15,77 & 69,26 & 61,83 \\
$(n=292)$ & $(18,43)$ & $(54,02)$ & $(2,74)$ & $(13,83)$ & $(22,05)$ \\
\hline 1993 & 59,38 & 153,56 & 18,24 & 60,93 & 53,83 \\
$(n=99)$ & $(26,14)$ & $(61,83)$ & $(1,15)$ & $(12,21)$ & $(15,94)$ \\
\hline Total & 55,95 & 125,61 & 15,58 & 66,45 & 65,89 \\
$(n=617)$ & $(20,33)$ & $(53,60)$ & $(2,94)$ & $(13,74)$ & $(27,69)$ \\
\hline
\end{tabular}
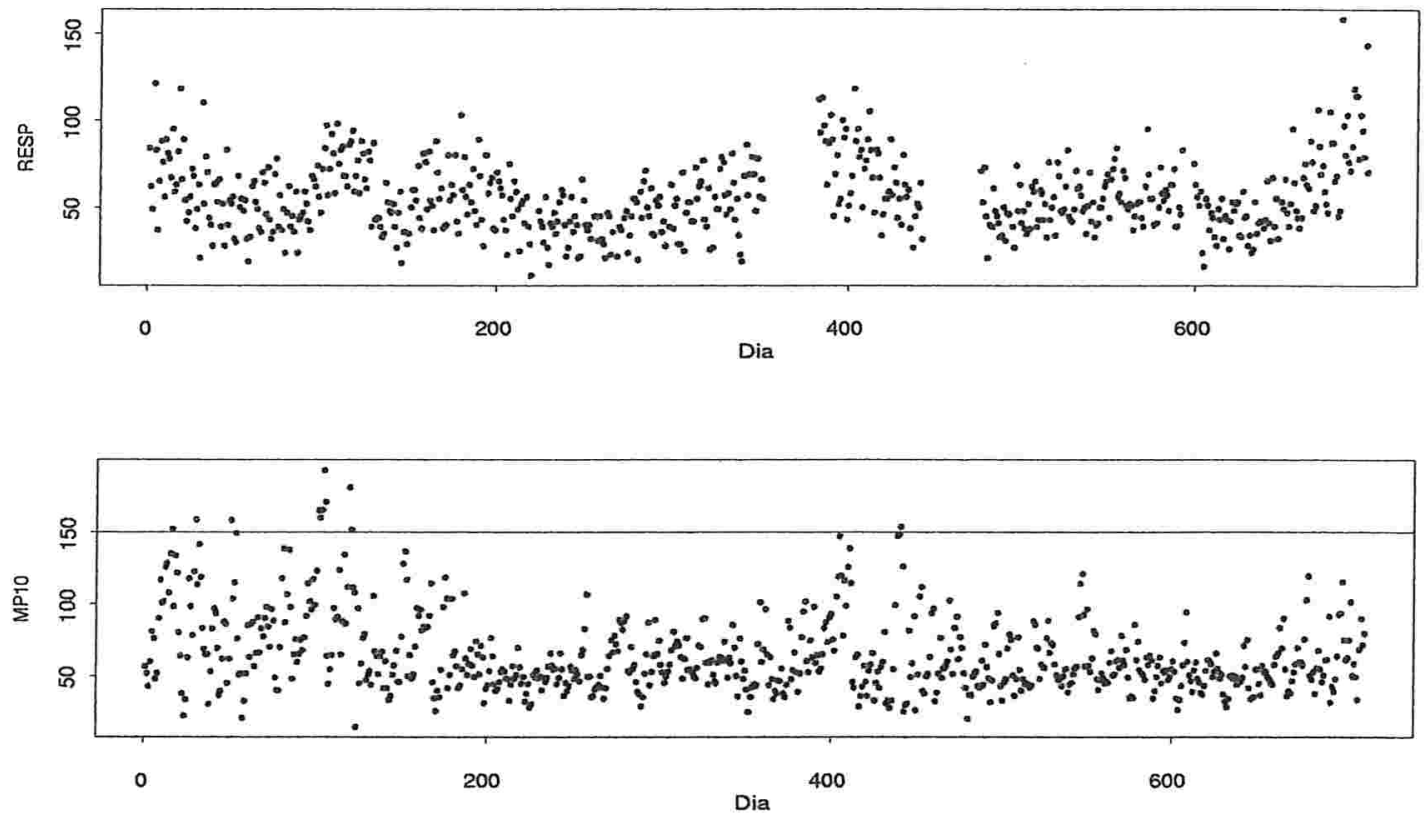

Figura 4.1. Séries de dados do número de visitas pediátricas de emergência por causas respiratórias (RESP) e da concentração de $\mathrm{MP}_{10}$ (com valor valor de referência internacional de qualidade do ar).

Considerando que a exposição ao poluente, à temperatura e à umidade deve preceder o efeito na saúde, incluímos médias móveis para cada uma dessas variáveis. A média móvel para uma variável é a média de seu valor no dia considerado com os dos dias precedentes, isto é, uma média móvel de dois dias é a média da variável no dia presente e no dia anterior. Médias móveis com defasagens de 1 a 7 dias foram avaliadas 
para cada uma das variáveis nos modelos log-lineares de Poisson e binomial negativo estudados inicialmente. Assim, na escolha do modelo básico foram avaliados 49 ajustes, e do modelo final 7 ajustes. Os menores valores da função de Akaike, em especial para o modelo de regressão de Poisson, maior significância das variáveis e, principalmente, melhor qualidade de ajuste do modelo determinaram a escolha das seguintes médias móveis: de 2 dias para a temperatura e de 7 dias para a umidade e a concentração de $\mathrm{MP}_{10}$, em ambos os modelos. O conjunto final de dados analisados contou com 611 observações. Logo, pela estratégia de análise proposta, inicialmente temos o modelo básico

$$
\begin{aligned}
\log [\mathrm{E}(\mathrm{RESP})]= & \beta_{1}+\beta_{2} \mathrm{Junho}_{1991}+\ldots+\beta_{23} \text { Abril }_{1993} \\
& +\beta_{24} \text { Segunda }+\ldots+\beta_{29} \text { Sabado } \\
& +\beta_{30} \mathrm{~T} .2+\beta_{31} \mathrm{U} .7+\beta_{32} \text { NRESP }
\end{aligned}
$$

onde RESP representa o número de atendimentos por causas respiratórias, T.2 a média móvel de 2 dias para temperatura, U.7 a média móvel de 7 dias para umidade e NRESP o número de atendimentos por causas não respiratórias; as demais variáveis são indicadoras (dummies). Uma vez avaliado o modelo básico, é feita a adição da variável explicativa MP10.7 a esse modelo, que representa a média móvel de sete dias para concentração de $\mathrm{MP}_{10}$, para avaliar se existe associação significativa entre a concentração do poluente e o número de atendimentos pediátricos de emergência por causas respiratórias após o controle das variáveis de confundimento.

Uma vez definida a estratégia de análise do problema sob investigação, primeiramente, consideramos o modelo de regressão log-linear de Poisson. Nesse modelo o estudo da significância de cada variável explicativa foi feito através do teste do desvio parcial (Seção 2.4). O modelo básico foi ajustado e associação significativa foi observada para todas as variáveis de confundimento $(p<0,001)$. A inclusão do poluente no modelo básico de Poisson é significante $(p<0,001)$. As estimativas dos parâmetros e respectivos erros padrão desse modelo estão apresentadas na Tabela 4.2. No entanto, o desvio desse modelo é igual a 1949,84 com 577 graus de liberdade (g.l.) indicando que o modelo de regressão de Poisson não fornece um bom ajuste. Além disso, todos os pontos do gráfico de probabilidade meio-normal dos resíduos do desvio (Figura 4.2a) estão acima do envelope simulado, levando à conclusão de que há fortes evidências de superdispersão.

Consideremos agora o modelo de regressão binomial negativo com função de ligação logarítmica e parâmetro $k$ desconhecido. Nesse modelo o estudo da significância de cada variável explicativa foi feito através do teste $\mathrm{F}$ (Seção 2.6). O modelo básico foi ajustado e associação significativa foi observada para todas as variáveis de confundimento ( $p<$ 0,001). O gráfico dos resíduos do desvio desse modelo básico contra o número de dias 
Tabela 4.2. Estimativas dos parâmetros do modelo log-linear de Poisson.

\begin{tabular}{|c|c|c|c|}
\hline Efeito & Parâmetro & Estimativa & Erro Padrão \\
\hline Intercepto & $\beta_{0}$ & 3,7914 & 0,1032 \\
\hline \multicolumn{4}{|l|}{ Mês/Ano } \\
\hline \multicolumn{4}{|l|}{ maio/1991 } \\
\hline junho/1991 & $\beta_{1}$ & $-0,2543$ & 0,0462 \\
\hline julho/1991 & $\beta_{2}$ & $-0,3324$ & 0,0465 \\
\hline agosto/1991 & $\beta_{3}$ & $-0,3488$ & 0,0453 \\
\hline setembro/1991 & $\beta_{4}$ & $-0,1259$ & 0,0455 \\
\hline outubro/1991 & $\beta_{5}$ & $-0,3457$ & 0,0461 \\
\hline novembro/1991 & $\beta_{6}$ & $-0,2056$ & 0,0464 \\
\hline dezembro/1991 & $\beta_{7}$ & $-0,3685$ & 0,0513 \\
\hline janeiro/1992 & $\beta_{8}$ & $-0,5168$ & 0,0515 \\
\hline fevereiro/1992 & $\beta_{9}$ & $-0,5389$ & 0,0517 \\
\hline março/1992 & $\beta_{10}$ & $-0,3855$ & 0,0490 \\
\hline abril/1992 & $\beta_{11}$ & $-0,2054$ & 0,0472 \\
\hline maio/1992 & $\beta_{12}$ & $-0,2344$ & 0,1416 \\
\hline junho/1992 & $\beta_{13}$ & 0,1282 & 0,0456 \\
\hline julho/1992 & $\beta_{14}$ & $-0,0937$ & 0,0458 \\
\hline agosto/1992 & $\beta_{15}$ & $-0,8764$ & 0,1821 \\
\hline setembro/1992 & $\beta_{16}$ & $-0,3453$ & 0,0500 \\
\hline outubro/1992 & $\beta_{17}$ & $-0,2196$ & 0,0463 \\
\hline novembro/1992 & $\beta_{18}$ & $-0,2336$ & 0,0469 \\
\hline dezembro/1992 & $\beta_{19}$ & $-0,2437$ & 0,0496 \\
\hline janeiro/1993 & $\beta_{20}$ & $-0,4120$ & 0,0521 \\
\hline fevereiro/1993 & $\beta_{21}$ & $-0,4303$ & 0,0524 \\
\hline março/1993 & $\beta_{22}$ & 0,0136 & 0,0478 \\
\hline abril/1993 & $\beta_{23}$ & 0,3578 & 0,0506 \\
\hline \multicolumn{4}{|l|}{ Dias da Semana } \\
\hline \multicolumn{4}{|l|}{ domingo } \\
\hline segunda-feira & $\beta_{24}$ & 0,5199 & 0,0333 \\
\hline terça-feira & $\beta_{25}$ & 0,6610 & 0,0279 \\
\hline quarta-feira & $\beta_{26}$ & 0,4922 & 0,0273 \\
\hline quinta feira & $\beta_{27}$ & 0,4813 & 0,0263 \\
\hline sexta-feira & $\beta_{28}$ & 0,4052 & 0,0254 \\
\hline sábado & $\beta_{29}$ & 0,2148 & 0,0229 \\
\hline Temperatura (T.2) & $\beta_{30}$ & 0,0170 & 0,0034 \\
\hline Ūmidade (U. $\overline{7}$ ) & $\beta_{31}$ & $-0,0045$ & 0,0009 \\
\hline Causas não respiratórias (NRESP) & $\beta_{32}$ & $-0,0017$ & 0,0002 \\
\hline Poluente MP 10 (MP10.7) & $\beta_{33}$ & 0,0047 & 0,0004 \\
\hline
\end{tabular}


(a)

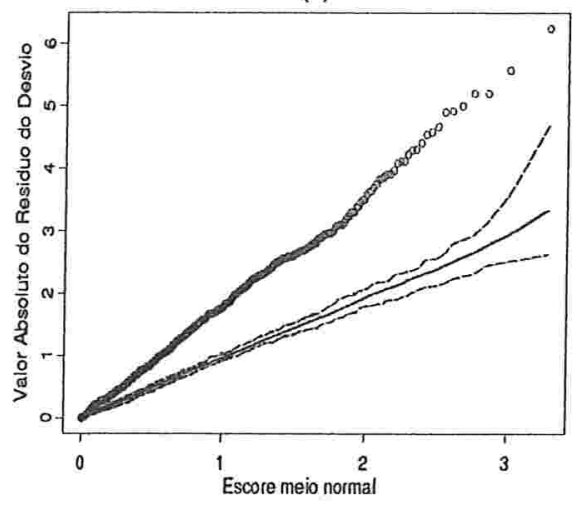

(b)

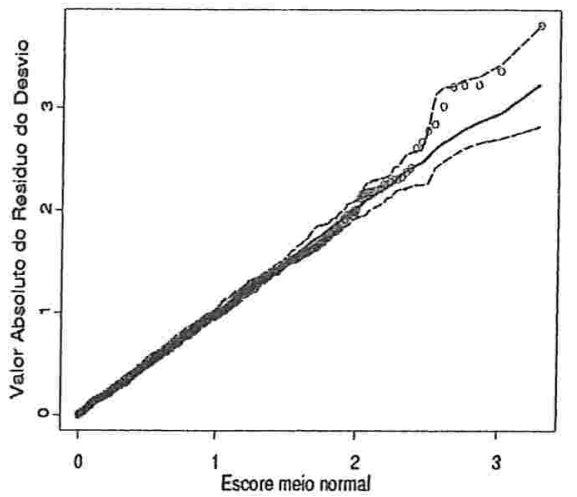

Figura 4.2. Gráfico de probabilidade meio-normal dos valores absolutos dos resíduos do desvio dos modelos de regressão de Poisson (a) e binomial negativo (b).

desde o início do estudo está apresentado na Figura 4.3a, juntamente com a curva de alisamento do tipo loess com parâmetros $\lambda=50 \%$ e $d=1$ (Seção 3.2). Essa curva está muito próxima de uma reta paralela ao eixo das abscissas, indicando que a tendência temporal dos dados parece controlada no modelo ajustado. Na Figura $4.3 \mathrm{~b}$ apresentamos o gráfico desses resíduos contra a média móvel de sete dias do poluente $\mathrm{MP}_{10}$ com a curva de alisamento do tipo loess. Essa curva sugere uma relação linear crescente do número de visitas pediátricas de emergência por causas respiratórias e a concetração do poluente, após o controle das variáveis de confundimento em estudo. A inclusão do poluente no modelo básico binomial negativo é significante $(p<0,001)$, o desvio desse modelo é igual a 625,35 com 577 g.l. e obtivemos $\widehat{k}=24,76$ com erro padrão igual a 2,10. As estimativas dos parâmetros de regressão e respectivos erros padrão desse modelo estão apresentadas na Tabela 4.3. O gráfico de probabilidade meio-normal dos resíduos do desvio (Figura 4.2b) indica que o modelo binomial negativo é muito mais apropriado para nossos dados que o modelo de Poisson.

(a)

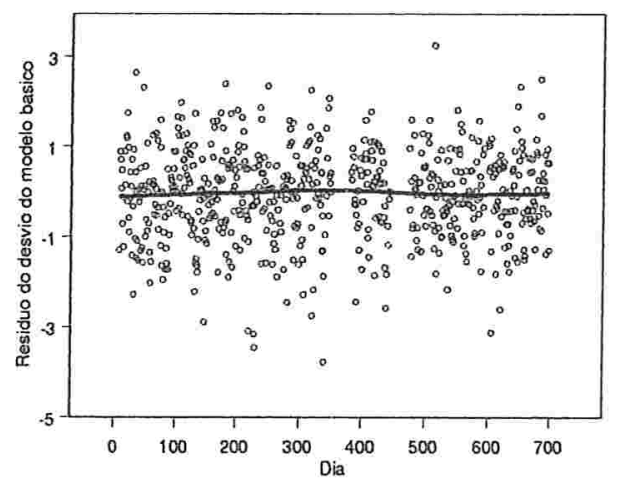

(b)

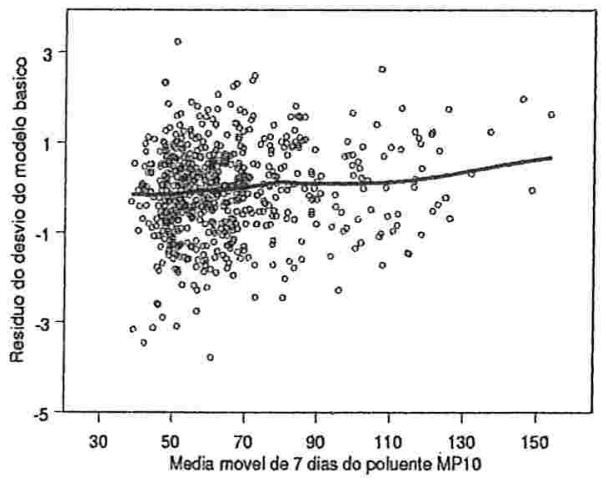

Figura 4.3. Gráfico dos resíduos do desvio do modelo binomial negativo básico contra os dias observados (a) e contra a média móvel de sete dias do poluente $\mathrm{MP}_{10}$ (b), com curvas de alisamento loess com parâmetros $\lambda=50 \%$ e $d=1$. 
Tabela 4.3. Estimativas dos parâmetros do modelo log-linear binomial negativo.

\begin{tabular}{|c|c|c|c|}
\hline Efeito & Parâmetro & Estimativa & Erro Padrão \\
\hline Intercepto & $\beta_{0}$ & 3,8421 & 0,1906 \\
\hline \multicolumn{4}{|l|}{ Mês/Ano } \\
\hline \multicolumn{4}{|l|}{ maio/1991 } \\
\hline junho/1991 & $\beta_{1}$ & $-0,2802$ & 0,0913 \\
\hline julho/1991 & $\beta_{2}$ & $-0,3537$ & 0,0905 \\
\hline agosto/1991 & $\beta_{3}$ & $-0,3761$ & 0,0893 \\
\hline setembro/1991 & $\beta_{4}$ & $-0,1423$ & 0,0908 \\
\hline outubro/1991 & $\beta_{5}$ & $-0,3716$ & 0,0900 \\
\hline novembro/1991 & $\beta_{6}$ & $-0,2158$ & 0,0912 \\
\hline dezembro/1991 & $\beta_{7}$ & $-0,3809$ & 0,0977 \\
\hline janeiro/1992 & $\beta_{8}$ & $-0,5211$ & 0,0969 \\
\hline fevereiro/1992 & $\beta_{9}$ & $-0,5561$ & 0,0975 \\
\hline março/1992 & $\beta_{10}$ & $-0,3953$ & 0,0941 \\
\hline abril/1992 & $\beta_{11}$ & $-0,2151$ & 0,0924 \\
\hline maio/1992 & $\beta_{12}$ & $-0,2506$ & 0,2568 \\
\hline junho/1992 & $\beta_{13}$ & 0,1166 & 0,0922 \\
\hline julho/1992 & $\beta_{14}$ & $-0,1147$ & 0,0908 \\
\hline agosto/1992 & $\beta_{15}$ & $-0,8972$ & 0,2813 \\
\hline setembro/1992 & $\beta_{16}$ & $-0,3593$ & 0,0955 \\
\hline outubro/1992 & $\beta_{17}$ & $-0,2253$ & 0,0905 \\
\hline novembro/1992 & $\beta_{18}$ & $-0,2406$ & 0,0917 \\
\hline dezembro/1992 & $\beta_{19}$ & $-0,2505$ & 0,0963 \\
\hline janeiro/1993 & $\beta_{20}$ & $-0,4207$ & 0,0989 \\
\hline fevereiro/1993 & $\beta_{21}$ & $-0,4322$ & 0,0991 \\
\hline março/1993 & $\beta_{22}$ & $-0,0133$ & 0,0950 \\
\hline abril/1993 & $\beta_{23}$ & 0,3546 & 0,1040 \\
\hline \multicolumn{4}{|l|}{ Dias da Semana } \\
\hline \multicolumn{4}{|l|}{ domingo } \\
\hline segunda-feira & $\beta_{24}$ & 0,5249 & 0,0598 \\
\hline terça-feira & $\beta_{25}$ & 0,6656 & 0,0506 \\
\hline quarta-feira & $\beta_{26}$ & 0,4944 & 0,0485 \\
\hline quinta feira & $\beta_{27}$ & 0,4923 & 0,0471 \\
\hline sexta-feira & $\beta_{28}$ & 0,4144 & 0,0448 \\
\hline sábado & $\beta_{29}$ & 0,2129 & 0,0390 \\
\hline Temperatura (T.2) & $\beta_{30}$ & 0,0156 & 0,0062 \\
\hline Umidade (U.7) & $\beta_{31}$ & $-0,0049$ & 0,0017 \\
\hline Causas não respiratórias (NRESP) & $\beta_{32}$ & $-0,0018$ & 0,0003 \\
\hline Poluente $\mathrm{MP}_{10}$ (MP10.7) & $\beta_{33}$ & 0,0048 & 0,0008 \\
\hline
\end{tabular}


O gráfico de resíduos de Pearson contra os preditores lineares (Figura 4.4a) não indica nenhuma evidência de falta de ajuste, e somente a observação 434 se destaca por apresentar maior valor que as demais, indicando que o número observado de visitas pediátricas de emergência foi muito maior que o esperado nesse dia. A Figura 4.4b apresenta um gráfico da distância de Cook para o ajuste binomial negativo. Observamos que a distância de Cook da observação 434 também se destaca das demais. A Figura 4.4c apresenta o gráfico de alavancas $h_{i}$ contra os valores ajustados. Observamos que os pontos com alta alavanca correpondem às únicas observações de maio e agosto de 1992 no nosso conjunto de dados, e essa é a razão pela qual elas são potencialmente influentes. No entanto, como a eliminação de todas essas observações discrepantes não altera as conclusões inferenciais da análise, elas não foram desconsideradas. A Figura 4.4d apresenta o gráfico da função de autocorrelação dos resíduos de Pearson, onde podemos observar que não há evidências de que a suposição de independência do modelo de regressão binomial negativo seja falsa.

(a)

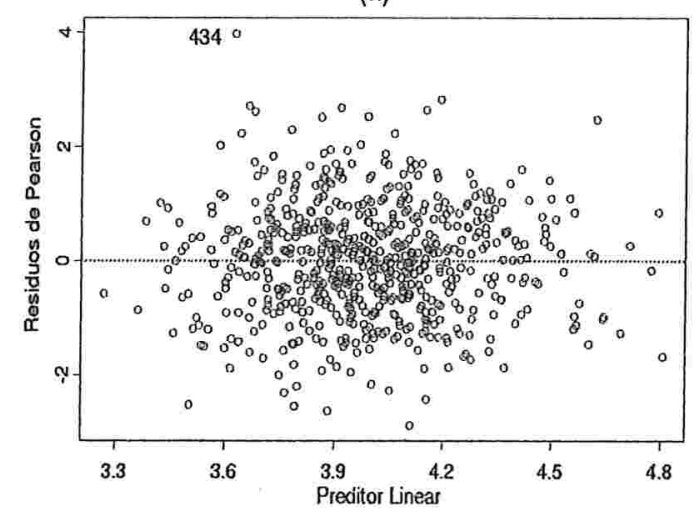

(c)

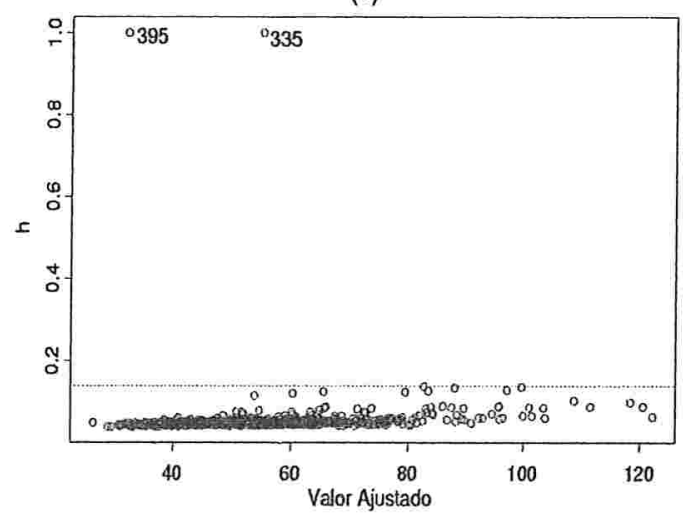

(b)

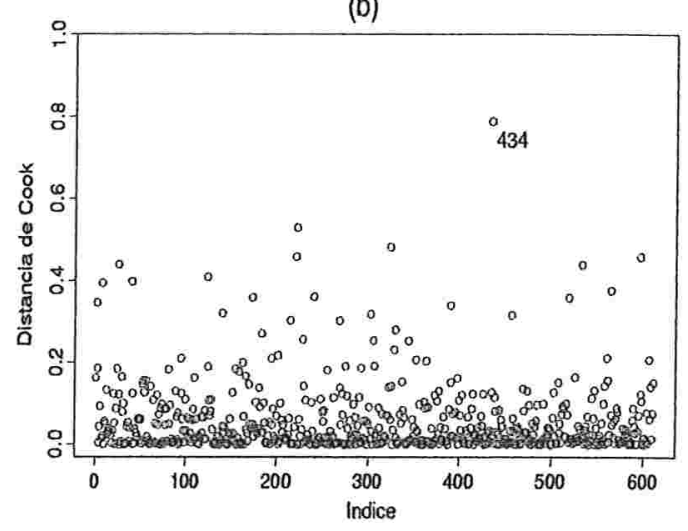

(d)

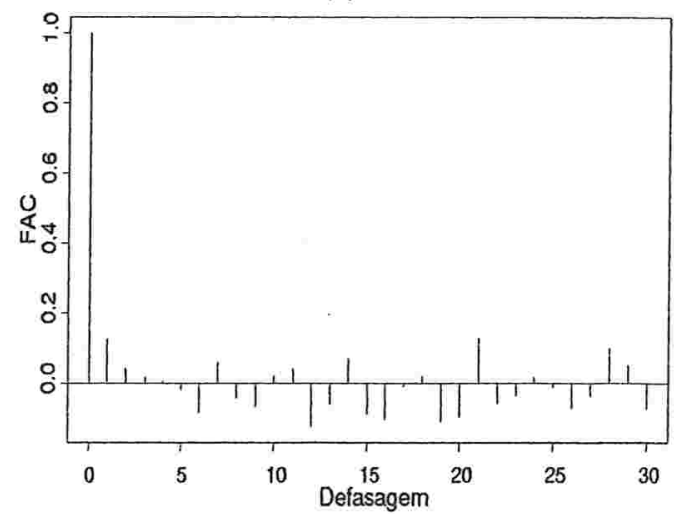

Figura 4.4. Gráfico dos resíduos de Pearson contra os valores ajustados (a), gráfico da distância de Cook (b), gráfico da medida de alavanca $h$ contra os valores ajustados (c) e gráfico da função de autocorrelação (FAC) dos resíduos de Pearson (d) para o modelo binomial negativo. 
As estimativas do parâmetro relacionado ao poluente $\mathrm{MP}_{10}$ em ambos os modelos log-lineares avaliados foram positivas (ver Tabelas 4.2 e 4.3 ) indicando que quanto maior a concentração desse poluente, maior será o valor esperado do número de atendimentos pediátricos de emergência diários por causas respiratórias. O risco relativo ao poluente $\mathrm{MP}_{10}$ que, como descrito na Seção 2.7, é uma medida de interesse em estudos epidemiológicos, e repectivo intervalo de $95 \%$ de confiança foram calculados para incrementos de até $70 \mu \mathrm{g} / \mathrm{m}^{3}$ na média móvel de sete dias do $\mathrm{MP}_{10}$, construindo assim a curva de risco relativo estimada, que está apresentada na Figura 4.5. Para efeito de comparação, foi incluída no gráfico a curva de risco relativo estimada através do modelo de Poisson. Apesar das estimativas pontuais dos riscos relativos calculados pelos dois modelos estarem muito próximas, os intervalos de confiança diferem consideravelmente de um modelo para outro. Isso ilustra perfeitamente o que foi discutido no Capítulo 1: uma conseqüência importante de se ignorar a superdispersão é que os erros padrão obtidos através do modelo de regressão de Poisson são incorretos e subestimam a variabilidade dos estimadores de seus parâmetros. O modelo de Poisson pode indicar falsa associação significante entre a resposta e as covariadas do modelo. Este é um exemplo de que conclusões erradas podem ser tiradas quando as suposições de um modelo teórico são violadas, e daí a importância de uma análise de diagnóstico para verificar a qualidade e a adequação do ajuste de um modelo estatístico. Esse fato não ocorreu nessa análise pela alta significância de todas as variáveis em estudo, não prejudicando assim os resultados apresentados em Lin et al. (1999).

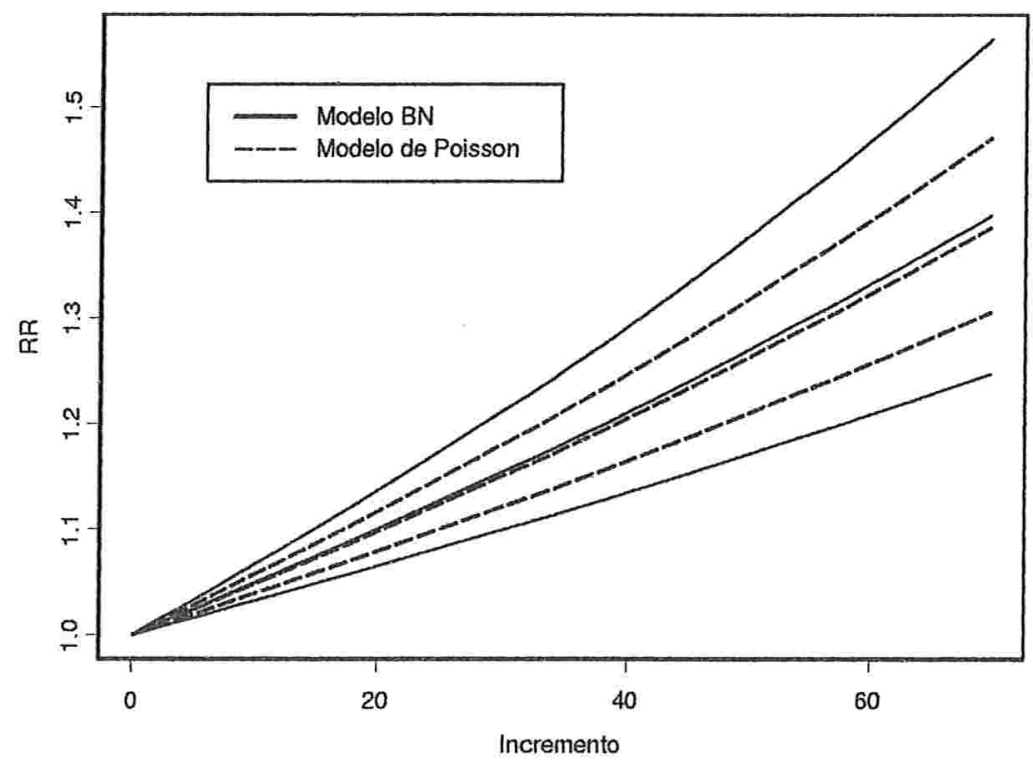

Figura 4.5. Estimativas pontuais das curvas de risco relativo (RR) associadas ao poluente $\mathrm{MP}_{10}$ e respectivos intervalos de $95 \%$ de confiança para os modelos binomial negativo (BN) e de Poisson. 
Para o modelo binomial negativo, as estimativas do risco relativo (intervalo de $95 \%$ de confiança) para o incremento de $10 \mu \mathrm{g} / \mathrm{m}^{3}$ na média móvel de sete dias do poluente e para o incremento da distância interquartílica ( $75-25$ percentil) dessa mesma média móvel que, nesse caso, é igual a $29,2 \mu \mathrm{g} / \mathrm{m}^{3}$, são $1,049([1,032 ; 1,066])$ e $1,150([1,097$; 1,205]) respectivamente. Essa última medida foi calculada com o objetivo de representar a razão do número esperado de atendimentos pediátricos de emergência por causas respiratórias em um dia de "muita" poluição comparado com um dia de "pouca" poluição. Assim, podemos dizer, por exemplo, que o aumento da distância interquartílica na média móvel de sete dias do poluente $\mathrm{MP}_{10}$ está associado a um aumento médio estimado de aproximadamente $15 \%$ no número de atendimentos pediátricos de emergência por causas respiratórias, considerando constantes as demais covariadas do modelo.

Finalmente vamos considerar o ajuste de um modelo aditivo binomial negativo com o mesmo objetivo de investigar a associação entre o número de atendimentos pediátricos de emergência por causas respiratórias e os níveis de poluição atmosférica na cidade de São Paulo. A proposta de análise apresentada anteriormente também será aplicada nesse ajuste, mas aqui, vamos considerar que a relação entre a variável resposta e as variáveis explicativas de confundimento, assim como o poluente, pode não apresentar forma paramétrica, e para avaliar tais relações propomos a inclusão de termos não paramétricos no modelo. Consideramos a função de ligação logarítmica. $\mathrm{O}$ método de suavização utilizado na estimação das funções não paramétricas foi o loess, e o procedimento para a escolha de seus parâmetros $\lambda$ e $d$ foi feito como descrito nas Seções 3.4 e 3.6, com especial atenção aos resultados gráficos.

O primeiro passo dessa análise foi modelar o efeito sazonal do número de atendimentos pediátricos de emergência, onde substituímos as variáveis indicadoras dos meses do período em estudo por uma função alisadora loess dos dias de observação (DIA), ou seja, propomos inicialmente o modelo

$$
\log [\mathrm{E}(\mathrm{RESP})]=\beta_{0}+\operatorname{loess}(\mathrm{DIA}, \lambda, d) .
$$

Para tal, foi necessário definir os parâmetros de alisamento da função loess com o auxílio de gráficos das curvas alisadas sobrepostas aos dados. Diversos parâmetros foram avaliados (Figura 4.6) e definimos os parâmetros $\lambda=25 \%$ e $d=2$ para o alisador loess em função dos dias de observação. Isto significa que a vizinhança em cada ponto deverá conter $25 \%$ das observações, o que corresponde a 153 dias (aproximadamente 6 meses). Note pela Figura 4.6 que a curva estimada no modelo aditivo binomial negativo com parâmetros $\lambda=15 \%$ e $d=2$ não elimina a variabilidade de forma satisfatória e aquela com parâmetros $\lambda=30 \%$ e $d=1$ o faz de forma exagerada. Assim, concluímos que os parâmetros $\lambda=25 \%$ e $d=2$ parecem controlar a sazonalidade e tendência de modo satisfatório, sem capturar a variabilidade total diária do número de atendimentos que desejamos explicar também através das demais variáveis confundidoras e da poluição. 
$\lambda=15 \%$ e $d=1$

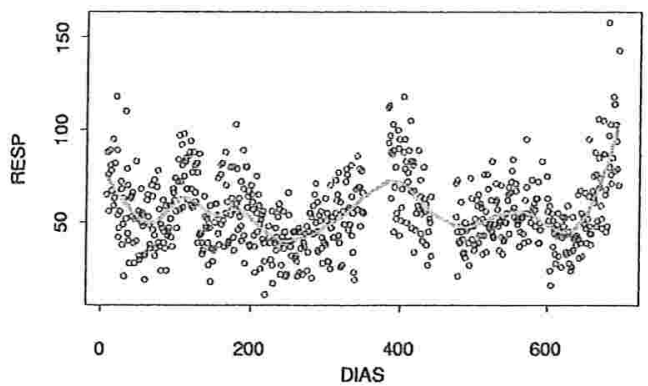

$\lambda=25 \%$ e $d=1$

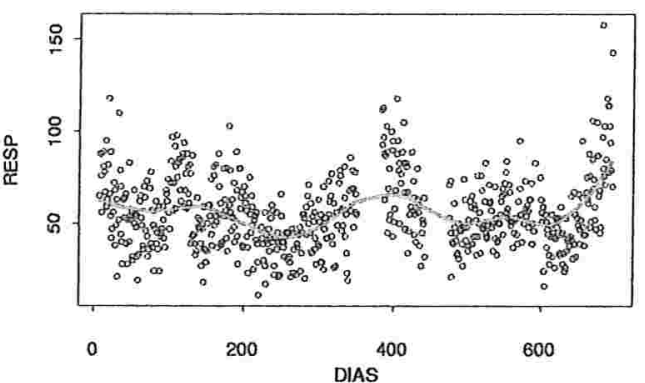

$\lambda=15 \%$ e $d=2$

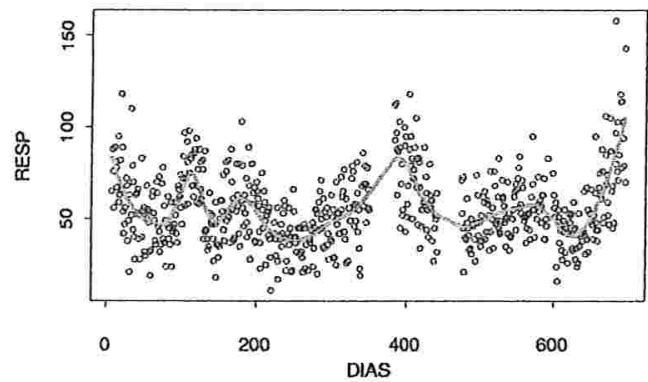

$\lambda=25 \%$ e d $=2$

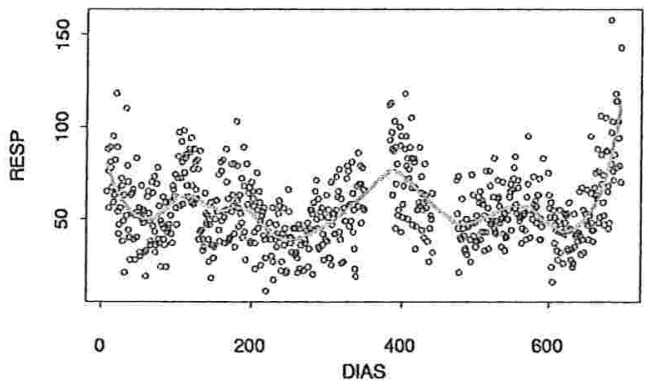

$\lambda=20 \%$ e $d=1$

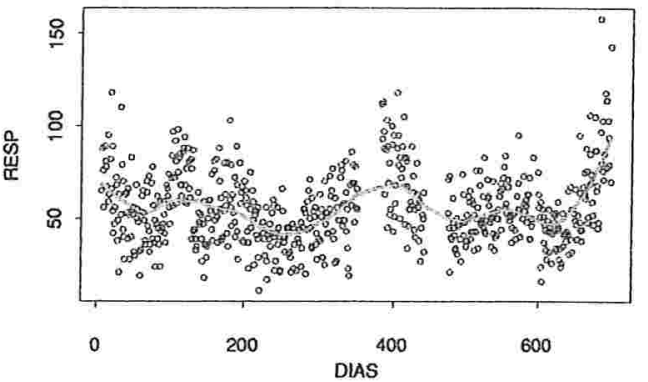

$\lambda=30 \%$ e $d=1$

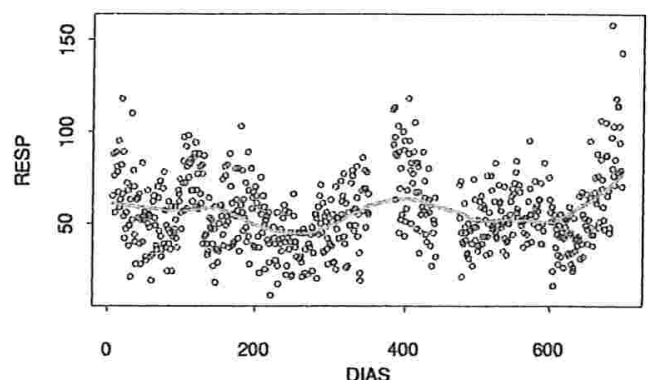

$\lambda=20 \%$ e $d=2$

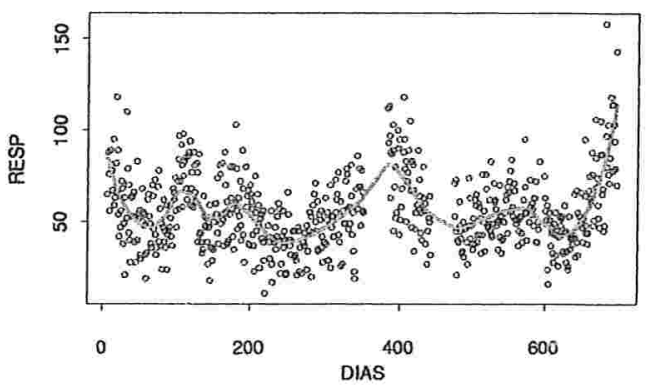

$\lambda=30 \%$ e $d=2$

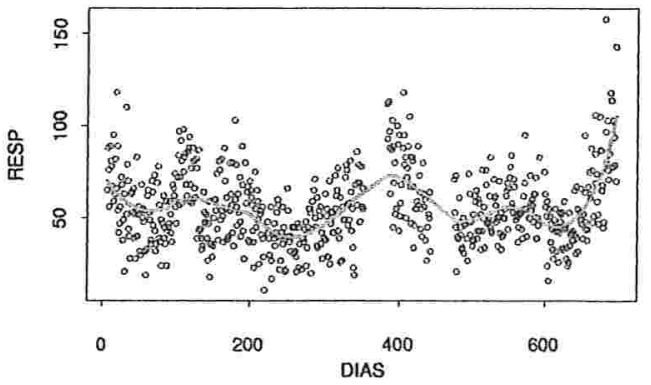

Figura 4.6. Valores ajustados pelo modelo aditivo binomial negativo com curvas alisadas do tipo loess $\operatorname{com} \lambda=50 \%$ e $d=1$ para o efeito sazonal com diferentes valores de $\lambda$ e $d$, sobrepostas aos dados. 
Vale destacar que esse modelo apresentou a menor soma dos quadrados das autocorrelações dos resíduos com defasagem de 1 a $30\left(\sum_{i=1}^{30} F A C_{i}^{2}=1,36\right)$, dentre todos avaliados, onde essa medida variou de 1,36 a 1,71. Para concluir a modelagem do efeito sazonal, incluímos nesse modelo as variáveis indicadoras do dia da semana, ou seja, temos o seguinte modelo de controle sazonal

$$
\log [\mathrm{E}(\mathrm{RESP})]=\beta_{0}+f_{1}(\mathrm{DIA}, \lambda=25 \%, d=2)+\beta_{2} \text { Segunda }+\ldots+\beta_{6} \text { Sabado. }
$$

O próximo passo dessa análise foi o controle das variáveis climáticas temperatura (T.2) e umidade (U.7) no modelo básico. Para avaliar a forma da associação entre cada uma dessas variáveis e o número de atendimentos de emergência já avaliado no modelo básico sazonal, fizemos, de forma intuitiva, gráficos das diferenças entre o preditor observado ( $\log (\mathrm{RESP})$ ) e a estimativa do preditor aditivo do modelo básico sazonal contra cada uma dessas variáveis (Figura 4.7). Aqui, nosso objetivo foi verificar a existência de alguma associação entre o "resíduo" do modelo básico sazonal e as variáveis climáticas, e para facilitar essa análise sobrepusemos aos dados uma curva não paramétrica do tipo loess $\operatorname{com} \lambda=50 \%$ e $d=1$ (parâmetros padrão) em cada gráfico. Observando essa figura temos evidências de uma associação próxima à linear tanto para a variável T.2 como para a U.7, sendo a primeira com tendência de crescimento e a segunda de decrescimento. Pelo teste $\mathrm{F}$ aproximado realizado para avaliar se a contribuição mais adequada de cada uma dessas variáveis no modelo básico sazonal é linear ou não paramétrica, concluímos, como esperado, pela não rejeição da hipótese de linearidade para ambos os parâmetros $\left(p_{\mathrm{T} .2}=0,204\right.$ e $\left.p_{\mathrm{U} .7}=0,272\right)$. Da mesma forma, através do teste $\mathrm{F}$ aproximado concluímos que a inclusão das variáveis meteorológicas no modelo básico sazonal foi significativa $(p<0,001)$. Assim, temos o seguinte modelo:

$$
\begin{aligned}
\log [\mathrm{E}(\mathrm{RESP})] & =\beta_{0}+f_{1}(\mathrm{DIA}, \lambda=25 \%, d=2)+\beta_{2} \text { Segunda }+\ldots+\beta_{6} \text { Sabado } \\
& =+\beta_{7} \mathrm{~T} .2+\beta_{8} \mathrm{U} .7 .
\end{aligned}
$$
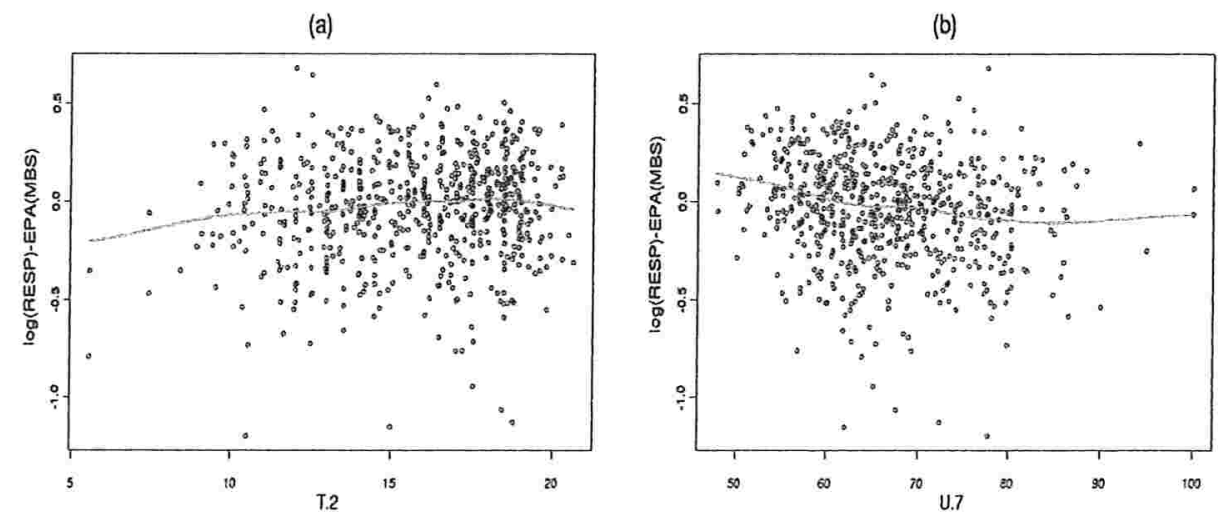

Figura 4.7. Gráfico da diferença entre o preditor observado $(\log ($ RESP)) e a estimativa do preditor aditivo (EPA) do modelo básico sazonal (MBS) contra a média móvel de dois dias para da temperatura (a) e contra a média móvel de sete dias da umidade (b), com curvas de alisamento loess $\operatorname{com} \lambda=50 \%$ e $d=1$. 
Para finalizar a construção do modelo básico, a inclusão da covariada número de atendimentos pediátricos de emergência por causas não respiratórias (NRESP) foi feita por essa mesma avaliação através de gráficos (Figura 4.8) e do teste $\mathrm{F}$ aproximado. Por esses resultados, temos que a inclusão dessa variável de forma não paramétrica apresentou melhores resultados no ajuste do modelo $(p<0,001)$. Avaliando os gráficos da Figura 4.8, onde temos curvas ajustadas com diversos parâmetros $\lambda$ e $d=1$ (o último foi fixado pelo comportamento dos dados), concluímos que os valores $\lambda=30 \% \mathrm{e}$ $d=1$ parecem controlar esses "resíduos" de modo satisfatório. Concluímos através do teste $\mathrm{F}$ aproximado que a inclusão da variável NRESP no modelo básico com controle sazonal e metereológico foi significativa $(p<0,001)$. Temos então o seguinte modelo básico completo:

$$
\begin{aligned}
\log [\mathrm{E}(\mathrm{RESP})] & =\beta_{0}+f_{1}(\mathrm{DIA}, \lambda=25 \%, d=2)+\beta_{2} \text { Segunda }+\ldots+\beta_{6} \text { Sabado } \\
& =+\beta_{7} \mathrm{~T} .2+\beta_{8} \mathrm{U} .7+f_{9}(\mathrm{NRESP}, \lambda=30 \%, d=1) .
\end{aligned}
$$
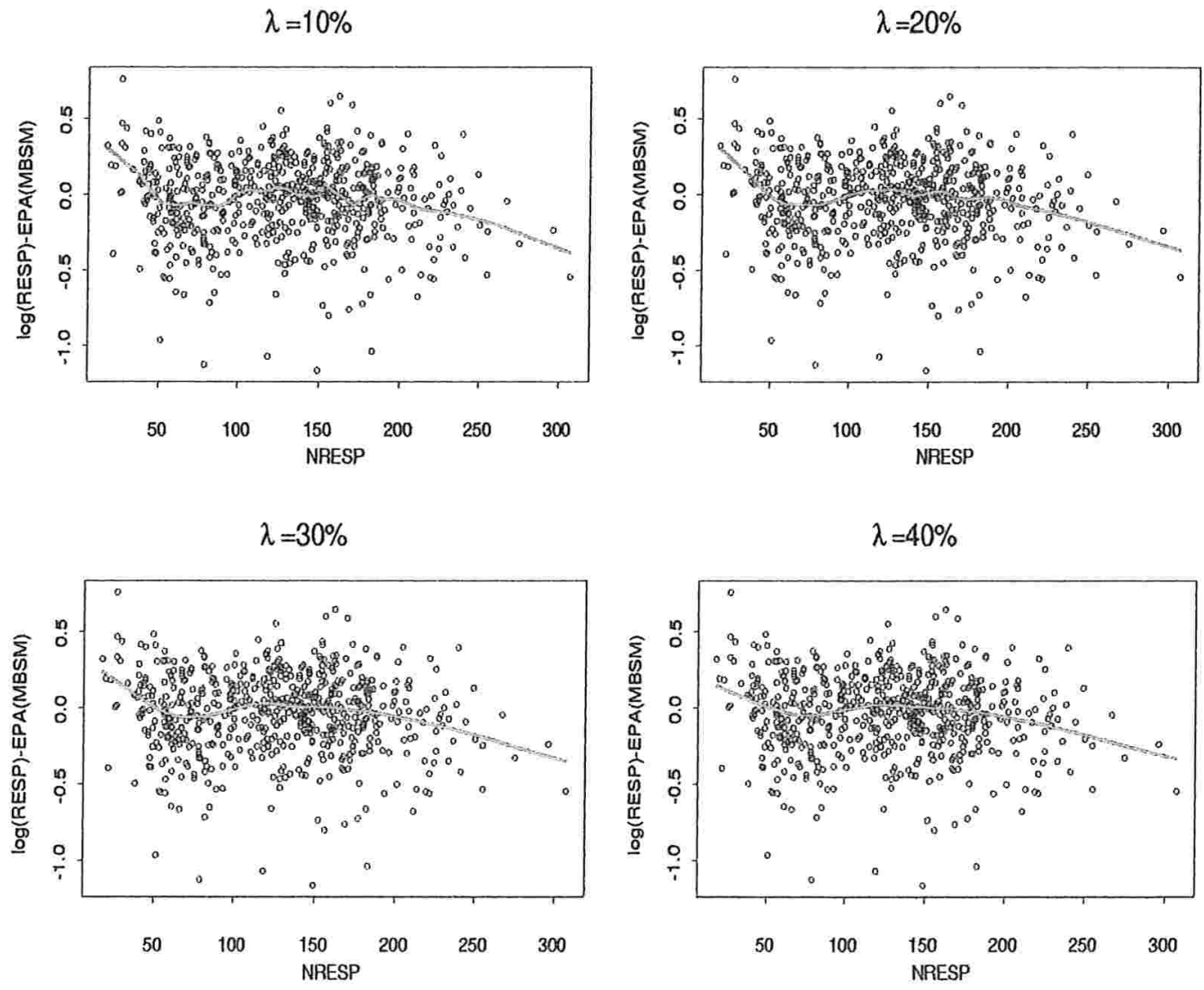

Figura 4.8. Gráfico da diferença entre o preditor observado $(\log (R E S P))$ e a estimativa do preditor aditivo (EPA) do modelo básico com controle sazonal e metereológico (MBSM) contra o número de atendimentos pediátricos de emergência por causas não respiratórias, com curvas de alisamento loess para diversos valores de $\lambda$ e $d=1$. 
Por fim, vamos avaliar a inclusão da média móvel de sete dias da concentração do poluente (MP10.7) no modelo básico completo. Inicialmente, essa avaliação também foi feita através de gráficos da diferença entre o preditor observado $(\log (\mathrm{RESP}))$ e a estimativa do preditor aditivo do modelo básico completo contra a média móvel de sete dias da concentração do poluente com diversos parâmetros $\lambda$ e $d=1$ (o último foi fixado pelo comportamento dos dados). Esses gráficos estão apresentados na Figura 4.9 , onde observamos que essa relação parece linear com leve tendência de crescimento. A curva com parâmetros $\lambda=45 \%$ e $d=1$ parece adequada para representar essa relação. $\mathrm{O}$ teste $\mathrm{F}$ aproximado para avaliar se a contribuição da covariada MP10.7 no modelo completo apresenta melhores resultados de forma linear ou não paramétrica foi realizado, e como esperado pela análise gráfica, não rejeitamos a primeira alternativa $(p=0,078)$. No entanto, como o nível de significância desse teste foi muito próximo de $5 \%$, e como um dos nossos objetivos com essa aplicação é ilustrar a curva de riscos relativos para o modelo aditivo binomial negativo, vamos propor o ajuste dos dois modelos

$$
\begin{aligned}
\log [\mathrm{E}(\mathrm{RESP})] & =\beta_{0}+f_{1}(\mathrm{DIA}, \lambda=25 \%, d=2)+\beta_{2} \text { Segunda }+\ldots+\beta_{6} \text { Sabado } \\
= & +\beta_{7} \mathrm{~T} .2+\beta_{8} \mathrm{U} .7+f_{9}(\mathrm{NRESP}, \lambda=30 \%, d=1) \\
= & +\beta_{10} \mathrm{MP} 10.7
\end{aligned}
$$

e

$$
\begin{aligned}
\log [\mathrm{E}(\mathrm{RESP})] & =\beta_{0}+f_{1}(\mathrm{DIA}, \lambda=25 \%, d=2)+\beta_{2} \text { Segunda }+\ldots+\beta_{6} \text { Sabado } \\
= & +\beta_{7} \mathrm{~T} .2+\beta_{8} \mathrm{U} .7+f_{9}(\mathrm{NRESP}, \lambda=30 \%, d=1) \\
= & +f_{10}(\mathrm{MP} 10.7, \lambda=45 \%, d=1) .
\end{aligned}
$$

Para esses dois modelos concluímos, através do teste $\mathrm{F}$ aproximado, que a inclusão da variável MP10.7 no modelo básico completo foi significativa $(p<0,001)$. Maiores detalhes desses dois ajustes estão apresentados na Tabela 4.4, onde temos estimativas dos parâmetros desses modelos muito próximas. Note que as estimativas do parâmetro relacionado ao poluente e respectivo erro padrão, obtidas no ajuste do modelo (4.1), são muito próximas das obtidas pelo modelo modelo log-linear binomial negativo (ver Tabelas 4.3 e 4.4), destacando que aqui também temos a primeira estimativa positiva. Vale lembrar que nos MAG's é possível representar graficamente o efeito estimado de cada variável explicativa do modelo na variável resposta, mantidas constantes as demais variáveis. No caso do modelo (4.2), a curva representada na Figura 4.10 é a contribuição estimada da MP10.7 no preditor aditivo ajustado, após controlar as variáveis confundidoras. As linhas pontilhadas são uma aproximação para o intervalo de confiança da curva de alisamento ajustada, e as barras verticais no eixo das abscissas representam os valores da MP10.7 efetivamente observados na amostra. Esta figura apresenta, como esperado, a curva ajustada próxima a uma reta com tendência de crescimento indicando, mais uma vez, que quanto maior a concentração desse poluente, 

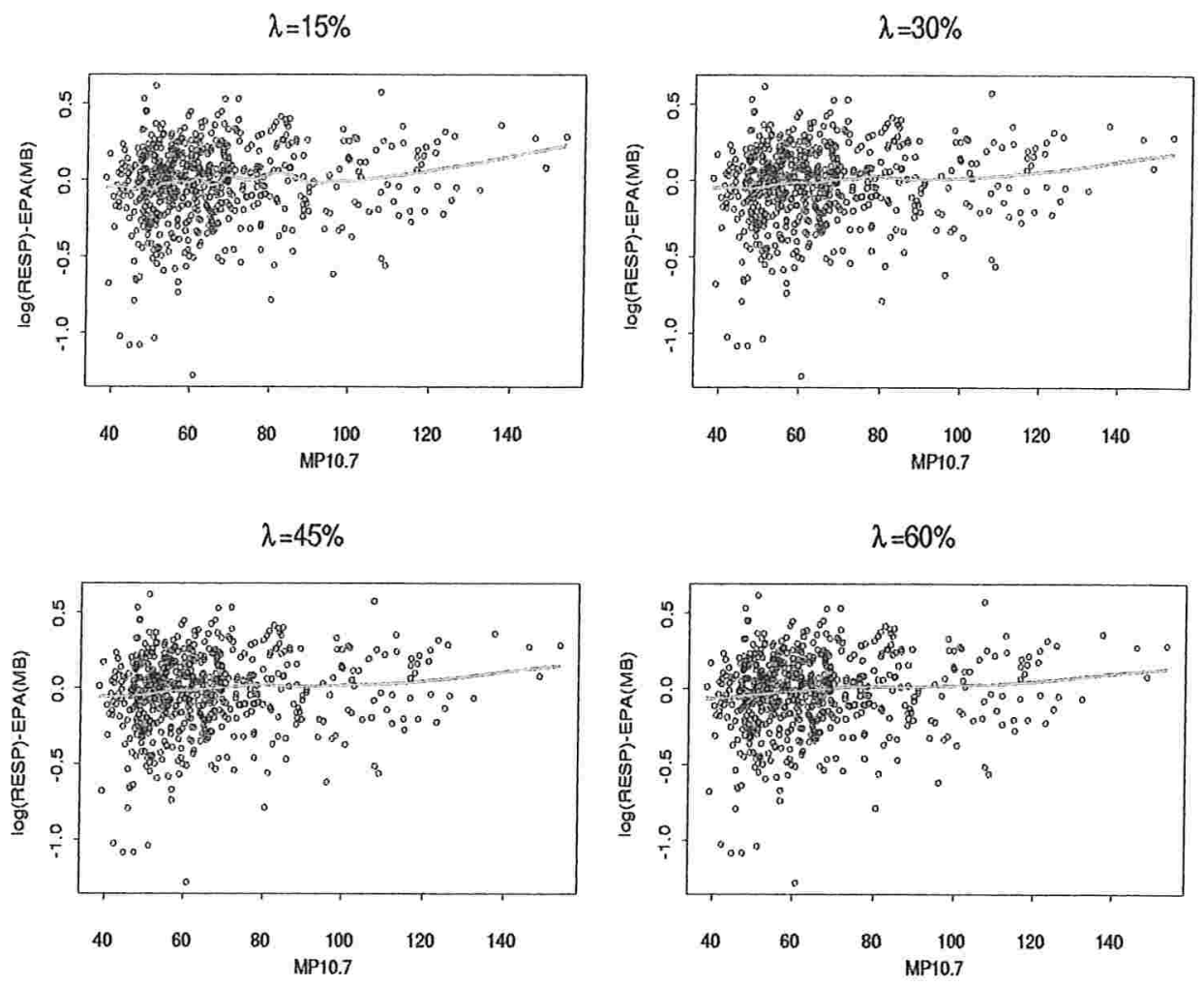

Figura 4.9. Gráfico da diferença entre o preditor observado (log(RESP)) e a estimativa do preditor aditivo (EPA) do modelo básico completo (MBC) contra a média móvel de sete dias da concentração do poluente, com curvas de alisamento loess para diversos valores de $\lambda$ e $d=1$.

maior será a estimativa desse termo não paramétrico e, logo, o valor esperado do número de atendimentos pediátricos de emergência diários por causas respiratórias.

A análise de diagnóstico desses modelos apresentou resultados muito similares (Figura 4.11). Os gráficos de probabilidade meio-normal dos resíduos do desvio apresentam poucos pontos acima do envelope, indicando que os modelos aditivos binomiais negativos propostos em (4.1) e (4.2) não parecem inadequados. Comparando esses dois gráficos com o gráfico de probabilidade meio-normal do modelo log-linear binomial negativo, apresentado na Figura $4.2 \mathrm{~b}$, concluímos que esses três modelos apresentam resultados muito similares quanto à qualidade de ajuste e parecem adequados para a análise proposta. Os demais gráficos da Figura 4.11 não indicam nenhuma evidência de falta de ajuste. 
Tabela 4.4. Estimativas dos parâmetros dos modelos aditivos binomiais negativos dados em (4.1) e (4.2).

\begin{tabular}{lcccc}
\hline & \multicolumn{2}{c}{ Modelo (4.1) } & \multicolumn{2}{c}{ Modelo (4.2) } \\
& Estimativa & Erro Padrão & Estimativa & Erro Padrão \\
\hline \hline Intercepto & 3,3683 & 0,1575 & 3,6631 & 0,1351 \\
\hline Dias da Semana & & & & \\
$\quad$ domingo & & & & \\
$\quad$ segunda-feira & 0,4598 & 0,0572 & 0,4700 & 0,0569 \\
$\quad$ terça-feira & 0,5792 & 0,0487 & 0,5902 & 0,0485 \\
$\quad$ quarta-feira & 0,4040 & 0,0468 & 0,4156 & 0,0466 \\
$\quad$ quinta feira & 0,4068 & 0,0454 & 0,4175 & 0,0452 \\
$\quad$ sexta-feira & 0,3475 & 0,0433 & 0,3564 & 0,0431 \\
$\quad$ sábado & 0,2303 & 0,0381 & 0,2326 & 0,0379 \\
\hline Temperatura (T.2) & 0,0124 & 0,0042 & 0,0101 & 0,0042 \\
Umidade (U.7) & $-0,0034$ & 0,0014 & $-0,0027$ & 0,0014 \\
Poluente MP MP10 $_{\text {(MP10.7) }}$ & 0,0047 & 0,0006 & - & - \\
\hline Dispersão & 27,2664 & 2,4003 & 27,9041 & 2,4794 \\
Desvio & 627,4090 & - & 628,2887 & - \\
Graus de Liberdade & 582,3740 & - & 578,4465 & - \\
\hline \hline
\end{tabular}

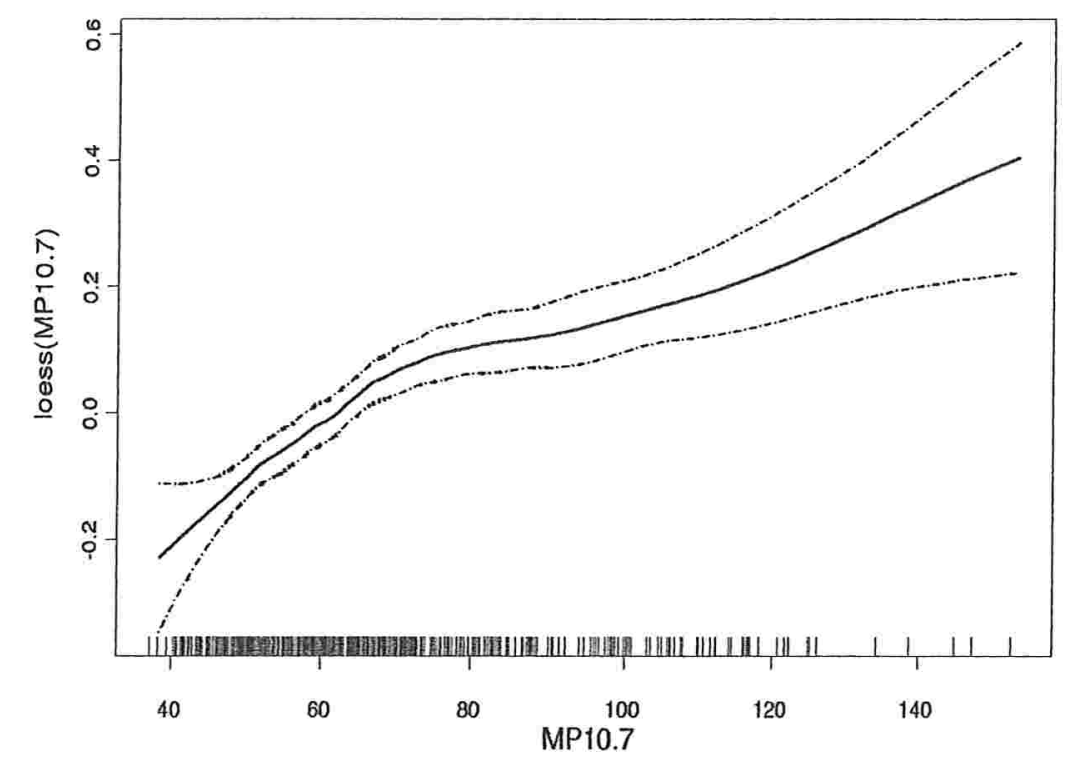

Figura 4.10. Curva estimada $\widehat{f}(\mathrm{MP} 10.7)=$ loess (MP10.7) no modelo aditivo binomial negativo completo, e respectivo intervalo de confiança aproximado. 

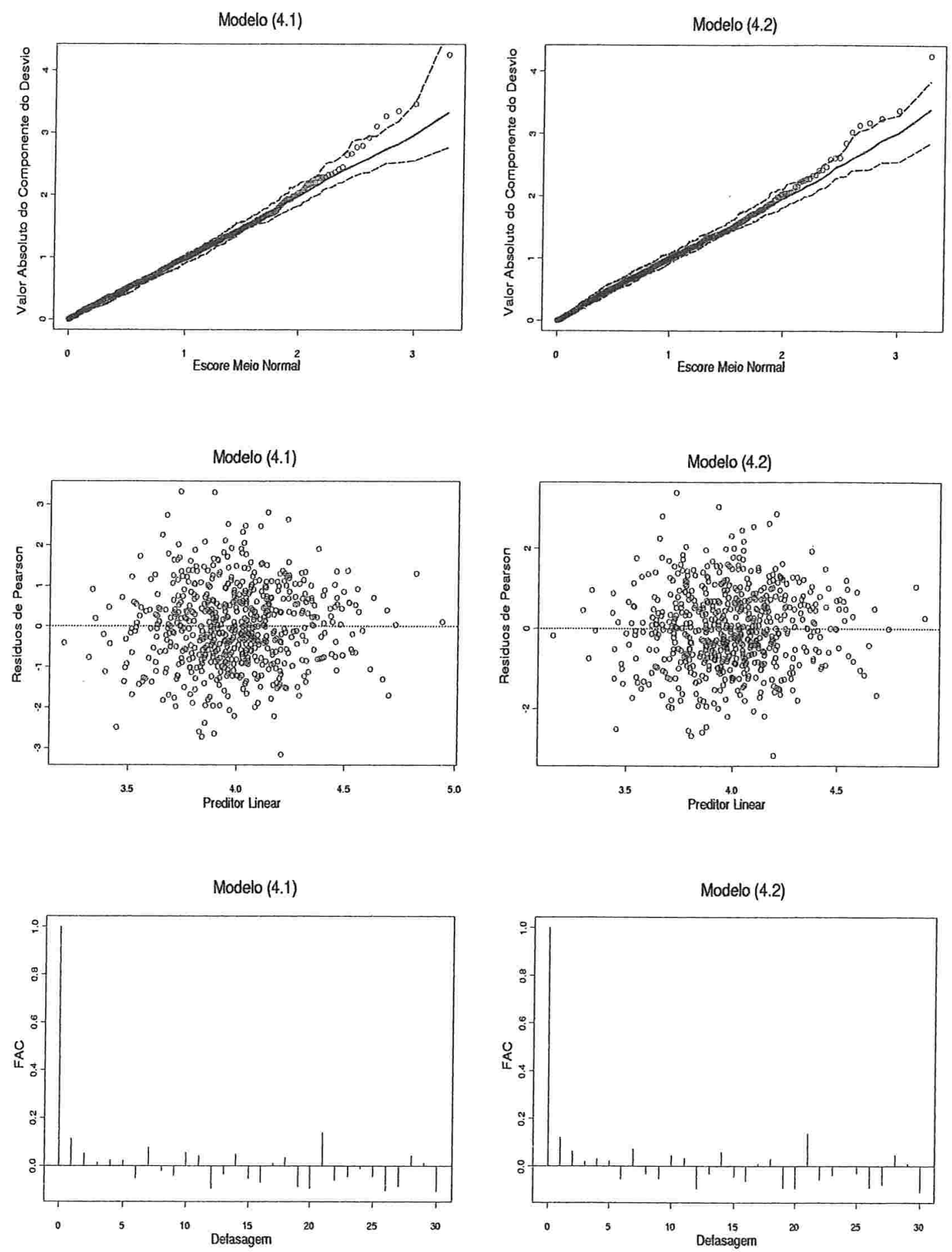

Figura 4.11. Gráficos de probabilidade meio-normal com envelope simulado dos valores absolutos dos resíduos componentes do desvio, dos resíduos de Pearson contra os valores ajustados e da função de autocorrelação (FAC) dos resíduos de Pearson para os modelos aditivos binomiais negativos dados em (4.1) e (4.2). 
Para o modelo aditivo binomial negativo (4.1), as estimativas do risco relativo (intervalo de $95 \%$ de confiança) para o incremento de $10 \mu \mathrm{g} / \mathrm{m}^{3}$ na média móvel de sete dias do poluente e para o incremento da distância interquartílica (75-25 percentil) dessa mesma média móvel são $1,049([1,036 ; 1,062])$ e 1, $149([1,108 ; 1,190])$ respectivamente. $\mathrm{O}$ risco relativo ao poluente $\mathrm{MP}_{10}$ e repectivo intervalo de $95 \%$ de confiança foram calculados para incrementos de até $70 \mu \mathrm{g} / \mathrm{m}^{3}$, construindo-se assim a curva de risco relativo estimada, que está apresentada na Figura 4.12. Para efeito de comparação, foi incluída no gráfico a curva de risco relativo estimada através do modelo log-linear binomial negativo, onde observamos resultados muito similares nas estimativas pontuais e intervalos de confiança aproximados de menor amplitude em relação aos obtidos pelos modelos aditivos.

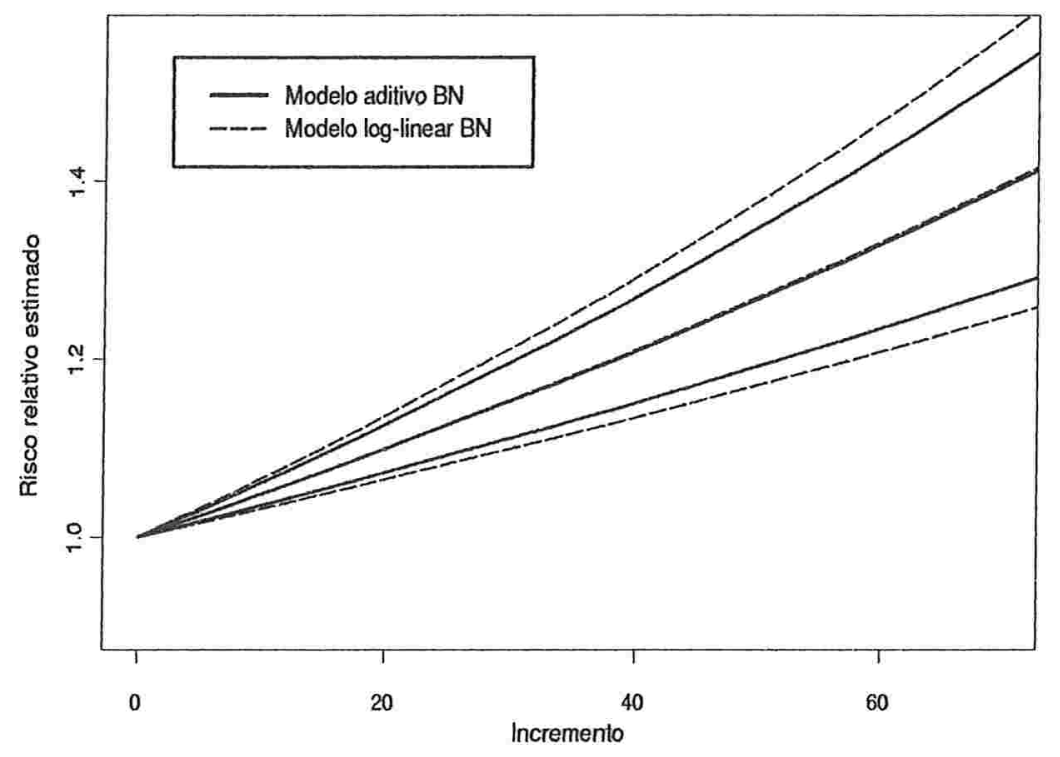

Figura 4.12. Estimativas pontuais das curvas de risco relativo associadas ao poluente $\mathrm{MP}_{10}$ e respectivos intervalos de $95 \%$ de confiança para os modelos log-linear e aditivo binomial negativo (BN).

As estimativas do risco relativo ao poluente para o modelo (4.2) foram avaliadas com valores de referência da variável explicativa MP10.7 iguais a $38,3 \mu \mathrm{g} / \mathrm{m}^{3}$ (valor mínimo) e $51,7 \mu \mathrm{g} / \mathrm{m}^{3}$ (primeiro quartil). A Figura 4.13 apresenta estimativas dessas curvas de risco, onde observamos que a escolha do valor de referência é muito importante, uma vez que produzem resultados muito diferentes. A estimativa do risco relativo de um determinado incremento no valor mínimo da média móvel de sete dias da concentração 
de $\mathrm{MP}_{10}$ é maior que a estimativa do risco relativo quando esse incremento é dado no primeiro quartil da MP10.7. Essa diferença no comportamento das curvas de risco relativo estimadas com diferentes valores de referência da MP10.7 nos chama a atenção para a importância da avaliação do impacto da poluição no número de atendimentos pediátricos de emergência com base em um valor de referência do poluente, o que na prática parece muito plausível. Parece razoável cosiderarmos que o risco relativo de atendimentos pediátricos de emergêcia para o incremento de $5 \mu \mathrm{g} / \mathrm{m}^{3}$ na média móvel de sete dias da concentração do $\mathrm{MP}_{10}$ seja diferente se nos referimos a uma mudança de, por exemplo, $40 \mu \mathrm{g} / \mathrm{m}^{3}$ para $45 \mu \mathrm{g} / \mathrm{m}^{3}$ ou de $110 \mu \mathrm{g} / \mathrm{m}^{3}$ para $115 \mu \mathrm{g} / \mathrm{m}^{3}$ na MP10.7. No entanto, esse fato não é considerado quando calculamos as estimativas dos riscos relativos a partir do ajuste de modelos log-lineares (ver Seção 2.7) e, portanto, pode ser visto com uma vantagem dos MAG's.

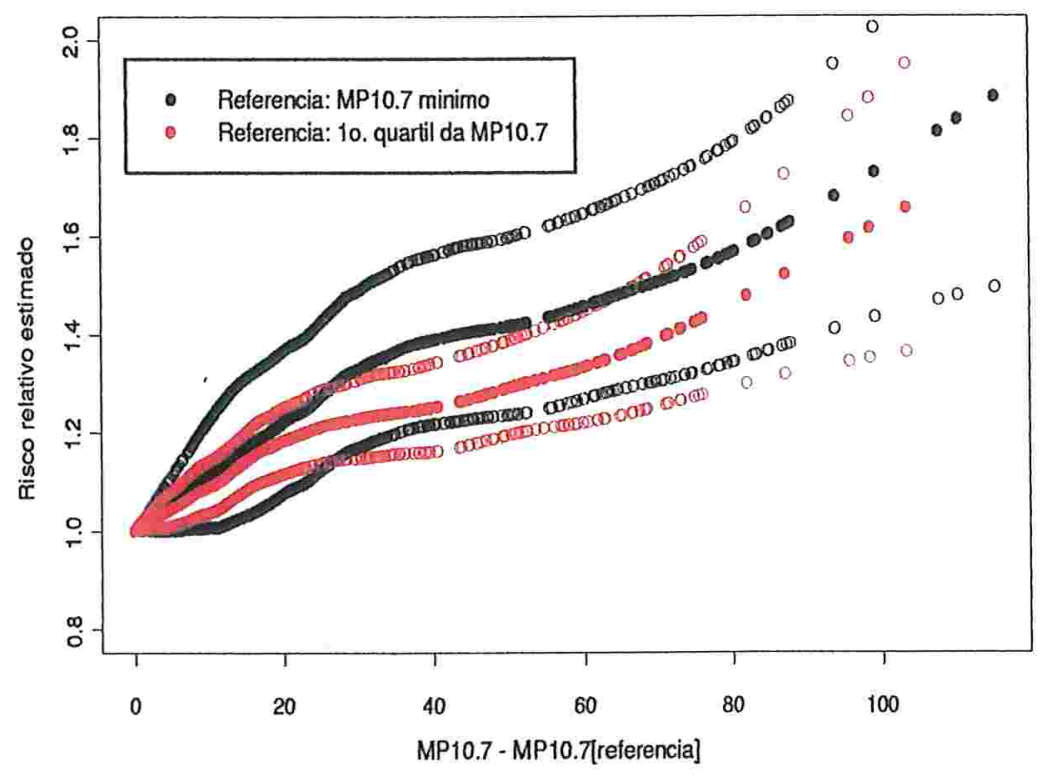

Figura 4.13. Curva de risco relativo estimada e respectivos intervalos de confiança para valores de referência do poluente $38,3 \mu \mathrm{g} / \mathrm{m}^{3}$ (mínimo) e $51,7 \mu \mathrm{g} / \mathrm{m}^{3}$ (primeiro quartil), através do modelo aditivo binomial negativo dado em (4.2).

Na Figura 4.14 temos uma comparação das curvas de risco relativo estimadas a partir de todos os modelos com distribuição binomial negativa estudados nesse capítulo, ou seja, o modelo log-linear (MLLBN) e os modelos aditivos (4.1) e (4.2) (MABN), sendo o último avaliado com os valores de referência mínimo e primeiro quartil da MP10.7. Observando essa figura, de uma forma geral, podemos dizer que o impacto do polu- 
ente no número de atendimentos pediátricos de emergência apresenta três diferentes comportamentos, o que não era de se esperar, uma vez que todos esses modelos ajustados, como mostramos ao longo dessa aplicação, parecem adequados para analisar os dados em estudo. Observamos também que a curva de risco relativo com referência no valor mínimo da MP10.7 apresenta os resultados mais distintos das demais e parece superestimar esses riscos. As demais curvas são próximas somente para incrementos maiores que aproximadamente $45 \mu \mathrm{g} / \mathrm{m}^{3}$, o que na prática geralmente, são resultados de menor interesse. Destacando assim, que temos grandes diferenças nos resultados de maior interesse, ou seja, para menores incrementos do poluente. Essa comparação está também ilustrada na Tabela 4.5, onde temos as estimativas e respectivos intervalos de confiança para incrementos de $10 \mu \mathrm{g} / \mathrm{m}^{3}$ e $50 \mu \mathrm{g} / \mathrm{m}^{3}$ na MP10.7 para cada modelo em estudo. Para incrementos de $10 \mu \mathrm{g} / \mathrm{m}^{3}$ essas estimativas variaram de 1,049 a 1,116 , o que parece uma variação importante do ponto de vista epidemiológico. Para incrementos de $50 \mu \mathrm{g} / \mathrm{m}^{3}$, o modelo aditivo (4.2) com referência no valor mínimo da MP10.7 apresentou a estimativa do risco igual a 1,417, que é maior que o limite superior dos intervalos de confiança das demais estimativas, onde temos resultados muito similares e em torno de 1,275 .

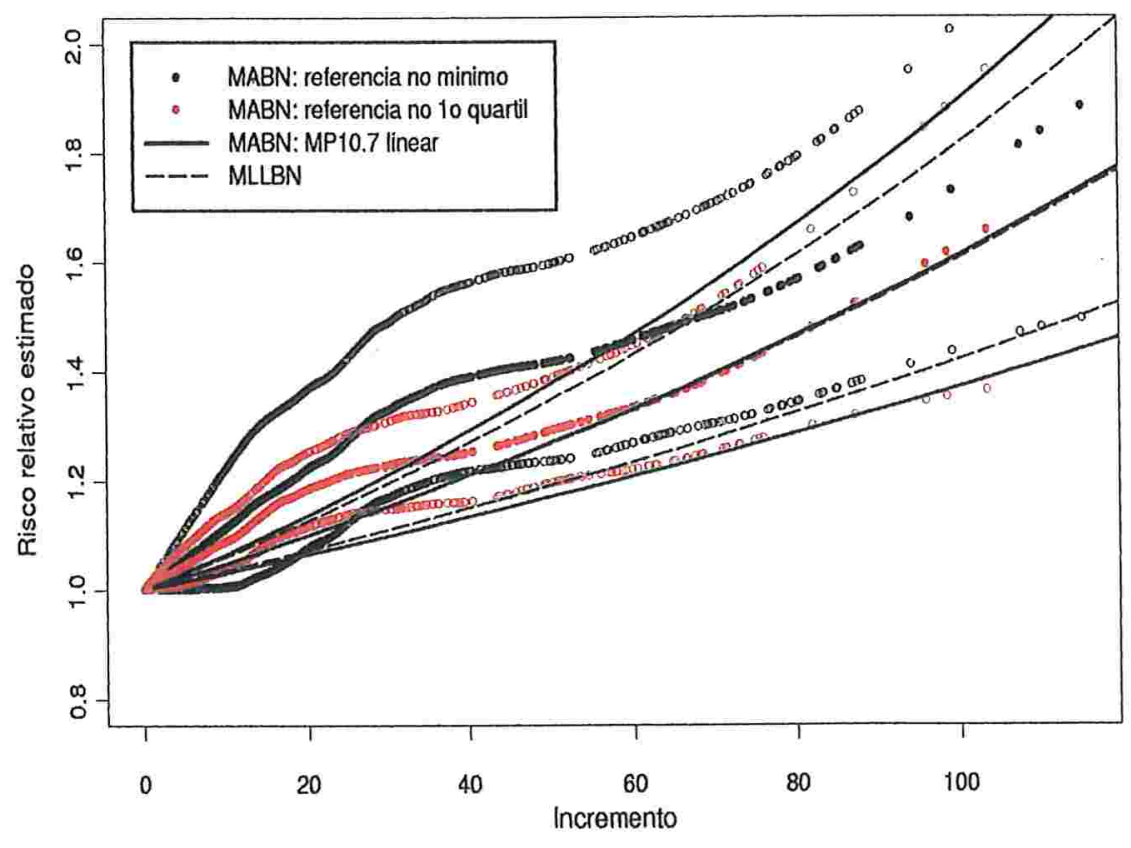

Figura 4.14. Comparação das curvas de risco relativo estimadas através dos diferentes modelos binomiais negativos desenvolvidos nesse estudo. 
Tabela 4.5. Estimativas do risco relativo (RR) associado com o poluente.

\begin{tabular}{cccccc}
\hline Modelos & \multicolumn{2}{c}{$\mathrm{RR}\left(10 \mu \mathrm{g} / \mathrm{m}^{3}\right)$} & & \multicolumn{2}{c}{$\mathrm{RR}\left(50 \mu \mathrm{g} / \mathrm{m}^{3}\right)$} \\
\cline { 2 - 3 } \cline { 5 - 6 } BN & 1,049 & {$[1,036 ; 1,062]$} & & 1,268 & {$[1,192 ; 1,348]$} \\
MLLimativa & $\mathrm{IC}(95 \%)$ & & Estimativa & $\mathrm{IC}(95 \%)$ \\
\hline MABN: MP10.7 linear & 1,049 & {$[1,032 ; 1,066]$} & & 1,270 & {$[1,171 ; 1,377]$} \\
MABN: ref. mínimo & 1,116 & {$[1,007 ; 1,225]$} & & 1,417 & {$[1,236 ; 1,597]$} \\
MABN: ref. 1o. quartil & 1,089 & {$[1,036 ; 1,141]$} & & 1,289 & {$[1,193 ; 1,385]$} \\
\hline
\end{tabular}

De qualquer forma, nossa análise baseada nos modelos binomiais negativos linear e aditivo, leva a evidências de associação positiva entre a concentração do poluente e o número de atendimentos após o controle de variáveis de confundimento. Este resultado reforça a idéia de que a poluição urbana é muito prejudicial à saúde identificando a necessidade de medidas eficientes de controle de emissão de poluentes visando proteger a saúde pública. 


\section{Capítulo 5}

\section{Conclusões}

- Os MLG's são uma ampla classe de modelos de regressão e apresentam métodos inferenciais e técnicas de diagnóstico bem desenvolvidos na literatura tanto do ponto de vista teórico como do prático. Os MAG's estendem a classe dos MLG's permitindo não linearidade na relação entre uma função da resposta média e as covariáveis, que é modelada através de funções alisadoras não especificadas, mas apresentam como desvantagens seu alto custo computacional e a falta de medidas de diagnóstico para a análise de pontos influentes e discrepantes. Comparando a aplicação dessas duas classes de modelos, podemos dizer que os MLG's enfatizam a estimação e análise inferencial dos parâmetros do modelo, enquanto os MAG's focalizam a exploração dos dados de forma não paramétrica, e são mais adequados para a exploração de conjuntos de dados e a visualização da relação entre as variáveis resposta e explicativas.

- Nosso estudo ilustra a importância de uma análise de diagnóstico, em particular para detectar uma possível inadequação do modelo de Poisson quando os dados revelam variabilidade maior do que a suposta por tal modelo. Uma conseqüência importante de se ignorar a superdispersão é que os erros padrão obtidos através do modelo de regressão de Poisson são incorretos e subestimam a variabilidade dos estimadores de seus parâmetros levando a intervalos de confiança enganosos. Assim, na presença de superdispersão o desvio do modelo de Poisson estará incorreto, e logo, teremos informações incorretas sobre a significância da cada parâmetro, podendo optar por um modelo complexo sem necessidade.

- Um dos modelos de superdispersão muito utilizado é o modelo com resposta binomial negativa. Se um certo parâmetro $(k)$ dessa distribuição é conhecido, ela faz parte da família exponencial e, logo, esse modelo per- 
tence à classe dos MLG's e dos MAG's. No entanto, a suposição de que tal parâmetro é conhecido é geralmente irreal e métodos adequados de estimação, inferência e diagnóstico na extensão desses modelos foram apresentados dos pontos de vista teórico e prático. Foi também desenvolvida a função gam.nb que possibilita a implementação computacional dos modelos aditivos binomiais negativos no software S-Plus. No entanto, a literatura estatística é pobre em estudos da validade das aproximações assintóticas para estimadores e testes nos MAG's. Assim, é mais prudente utilizar os MAG's e os modelos aditivos binomiais negativos para análises exploratórias do que com propósitos inferencias.

- A seleção de modelos binomiais negativos é uma tarefa difícil pela falta de medidas de qualidade do ajuste adequadas à presença de um certo parâmetro $(k)$ que apresenta variação de modelo a modelo. Concluímos que a avaliação gráfica dos dados e a aplicação de técnicas de diagnóstico nos diferentes modelos podem ser alternativas para a seleção de um modelo mais adequado, ressaltando que a primeira alternativa pode ser muito subjetiva.

- Utilizamos modelos binomiais negativos para a análise de associação entre o número de atendimentos pediátricos de emergência diários por motivos respiratórios registrados no Instituto da Criança da USP, em São Paulo, e os níveis de poluição atmosférica, representados pela concentração de material particulado $\left(\mathrm{MP}_{10}\right)$, no período de maio 1991 a abril de 1993. Mostramos que esses modelos são bem mais adequados que o modelo de regressão usual de Poisson para o conjunto de dados considerado. Nossa análise leva a evidências de associação positiva entre a concentração do poluente e o número de atendimentos após o controle de variáveis de confundimento, reforçando a idéia de que a poluição urbana é muito prejudicial à saúde identificando a necessidade de medidas eficientes de controle de emissão de poluentes visando proteger a saúde pública.

- A interpretação dos resultados dos modelos estudados através de estimativas do risco relativo deve ser vista com muito cuidado, uma vez que vários modelos bem ajustados apresentaram resultados muito diferentes para essa medida. Os riscos relativos calculados a partir dos MAG's apresentam como importante vantagem do ponto de vista epidemiológico a dependência de um valor de referência da covariável relacionada que, no caso da nossa aplicação, é representada pela concentração do poluente. 


\section{Apêndice A}

\section{Função gam.nb}

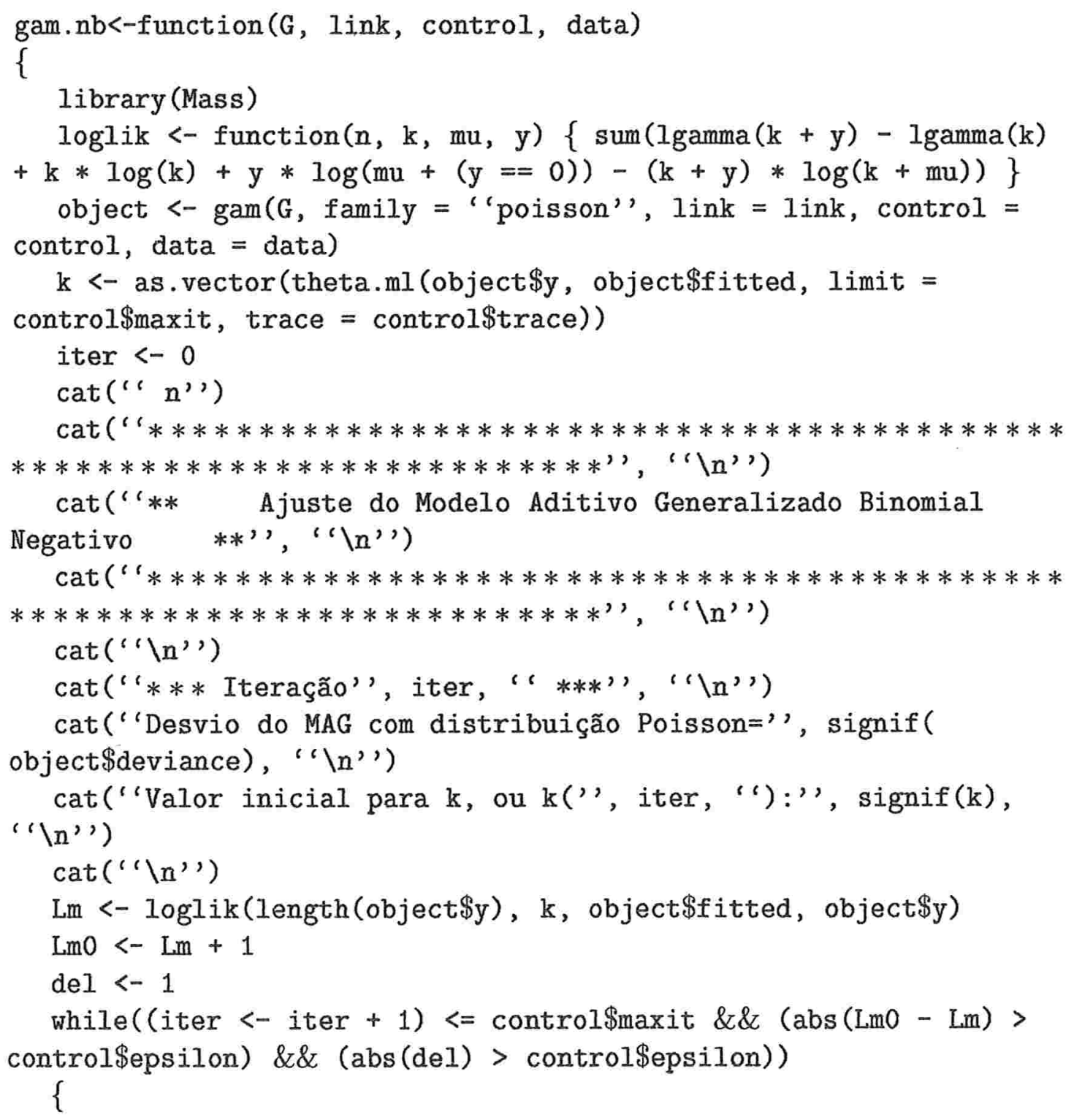




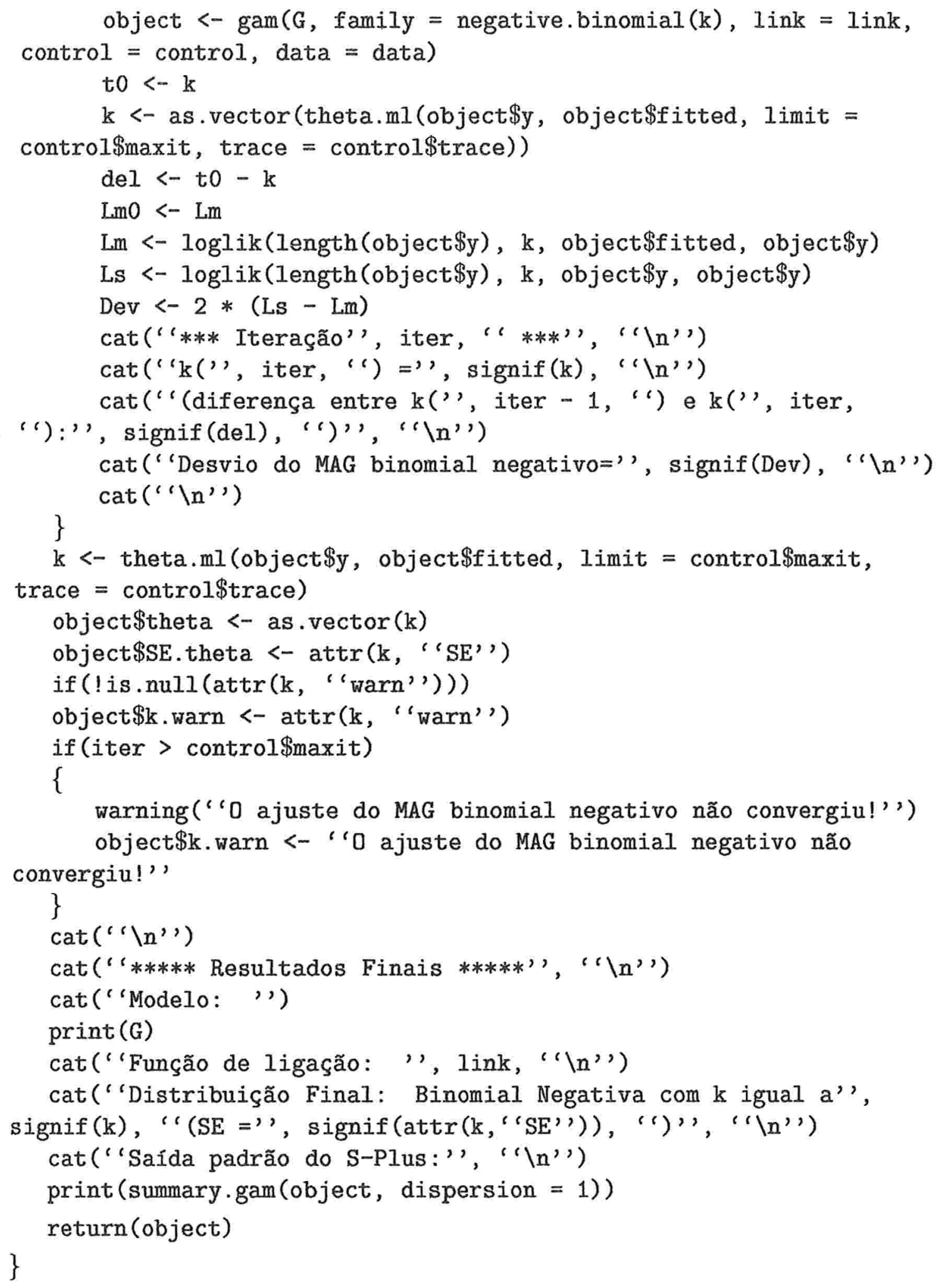




\section{Referências Bibliográficas}

[1] Akaike, H. (1974). A new look at statistical model identification. IEEE Transactions on Automatic Control, AU-19, 716-722.

[2] Atkinson, A. (1985). Plots, Transformations and Regression. Oxford: Clarendon Press.

[3] Braga, A.L.F, Conceição, G.M.S., Pereira L.A.A.; Kishi, H., Pereira, J.C.R., Andrade, M.F., Gonçalves, F.L.T., Saldiva,P.H.N. e Latorre, M.R.D.O. (1999). Air Pollution and pediatric respiratory admissions in São Paulo, Brazil. Journal of Environmental Medicine, 1, 95-102.

[4] Braga, A.L.F., Saldiva, P.H.N., Pereira, L.A.A., Menezes, J.J.C., Conceição, G.M.S., Lin, C.A., Zanobetti, A., Schwartz, J. e Dockery, D.W. (2001). Health effects of air pollution exposure on children and adolescents in São Paulo, Brazil. Pediatric Pulmonology, 31, 106-113.

[5] Breslow, N. (1984). Extra-poisson variation in log-linear models. Applied Statistics, 33, 38-44.

[6] Buja, A., Hastie, T.J. e Tibishirani, R.J. (1989). Linear smoothers and additive models. The Annals of Statististics, 17, 453-555.

[7] CETESB (2001). Relatório de Qualidade do Ar no Estado de São Paulo. São Paulo, CETESB.

[8] Chambers, J.M. e Hastie, T.J. (1993). Statistical Models in S. New York: Chapman \& Hall.

[9] Cleveland, W.S. (1979). Robust locally weighted regression and smoothing scarterplots. Journal of the American Statistical Association, 74, 829-836.

[10] Conceição, M.S.C., Miraglia, S.G.E.K., Kishi, H.S., Saldiva, P.H.N. e Singer, J.M. (2001). Air pollution and child mortality: a time-series study in São Paulo, Brazil. Environmental Health Perspectives, 109, 347-350. 
[11] Cook, R.D. (1986). Assessment of local influence (with discussion). Journal of the Royal Statistical Society B, 48, 133-169.

[12] David, J.S.E. (1999). Regressão Logística, Regressão de Poisson e Modelos Lineares Generalizados. Projeto de Iniciação Científica, IME-USP. Não publicado.

[13] David, J.S.E. (2000). Modelos de Superdispersão para Dados de Contagem. Projeto de Iniciação Científica, IME-USP. Não publicado.

[14] Dean, C. (1992). Testing overdispersion in poisson and binomial regression models. Journal of the American Statistical Association, 87, 451-457.

[15] Demétrio, C.G.B. (2001). Modelos Lineares Generalizados em Experimentação Agronômica. ESALQ-USP. Disponível em http://ce.esalq.usp.br/dce/clarice/Apostila.pdf.

[16] Ferrari, S.L.P. David, J.S.E, André, P.A. e Pereira, L.A.A. (2002). Overdispersed Regression Models for Air Pollution and Human Health. Em: Statistical Data Analysis Based on the $L_{1}$-Norm and Related Methods (Statistics for Industry and Technology, Y. Dodge (Editor), 429-438. Basel: Birkhäuser.

[17] Fox, J. (2000). Nonparametric Simple Regression: Smothing Scatterplots. Sage University Papers Series on Quantitative Applications in the Social Sciences, 07-130. Thousand Oaks, CA: Sage.

[18] Hardin, J. e Hilbe, J. (2001). Generalized Linear Models and Extensions. College Station: Stata Press.

[19] Hastie, T.J. e Tibshirani, R.J. (1986). Generalized additive models. Statistical Science, 3, 297-318.

[20] Hastie, T.J. e Tibshirani, R.J. (1987). Generalized additive models: some applications. Journal of the American Statistical Association, 82, 371-386.

[21] Hastie, T.J. e Tibshirani, R.J. (1990). Generalized Additive Models. New York: Chapman \& Hall.

[22] Hinde, J. e Demétrio, C. (1998). Overdispersion: models and estimation. Computational Statistics and Data Analysis, 27, 151-170.

[23] Hoaglin, D.C. e Welsch, R.E. (1978). The hat matrix in regression and ANOVA. The American Statistician, 32, 17-22. 
[24] Johnson, N.L., Kotz, S. e Kemp, A.W. (1993). Univariate Discrete Distributions. 2a edição. New York: John Wiley \& Sons.

[25] Kim, C., Park, B.U. e Kim, W. (2002). Influence diagnostics in semiparametric regression models. Statistics \& Probability Letters, 60, 49-58.

[26] Lambert, D. e Roeder, K. (1995). Overdispersion diagnostics for generalized linear models. Journal of the American Statistical Association, 95, 12251237.

[27] Lawless, J. (1987). Negative binomial and mixed Poisson regression. The Canadian Journal of Statistics, 15, 209-225.

[28] Lima, L.P. (2001). Modelos Aditivos Generalizados: Aplicação a um Estudo Epidemiológico Ambiental. Dissertação de Mestrado: Instituto de Matemática e Estatística da Universidade de São Paulo.

[29] Lin, C.A., Martins, M.A., Farhat, S.C., Pope III, C.A., Conceição, G.M.S., Anastasio, V.M., Hatanaka, M., Andrade, W.C., Hamaue, W.R., Böhm, G.M. e Saldiva, P.H.N. (1999). Air pollution and respiratory illness of children in São Paulo, Brazil. Paediatric and Perinatal Epidemiology, 13, 475488.

[30] Marsh, L.C. e Cormier, D.R (2001). Spline Regression Models. Sage University Papers Series on Quantitative Applications in the Social Sciences, 07-130. Thousand Oaks, CA: Sage.

[31] McCullagh, P. e Nelder, J.A. (1989).Generalized Linear Models (The Monographs on Statistics and Applied Probability, Vol 37), 2a edição. London: Chapman and Hall.

[32] Moore, D. (1986). Asymptotic properties of moment estimates for overdispersed counts and proportions. Biometrika, 73, 583-588.

[33] Morettin, P.A. e Toloi, C.M.C. (1985). Previsão de Séries Temporais. São Paulo: Atual.

[34] Neter, J., Kutner, M.H., Nachtsheim, C.J. e Wasserman, W. (1996). Applied Linear Statistical Models (Irwin Series in Statistics), 4a edição. Chicago: Irwin.

[35] Opsomer, J.D. (2000). Assymptotic properties of backfitting estimators. Journal of Multivariate Analysis, 73, 166-179. 
[36] Opsomer, J.D. e Ruppert, D. (1999). A root-n consistent estimators for semiparametric additive models. Journal of Computational and Graphical Statistics, 8, 715-732.

[37] Paul, S., Liang, K. e Self, S. (1989). On testing departure from binomial and multinomial assumptions. Biometrics, 45, 231-236.

[38] Paula, G.A. (2002). Modelos de Regressão. IME-USP. Disponível em http://www.ime.usp.br/ giapaula/Book.pdf.

[39] Pedan, A. (2001). Analysis of count data using the SAS system. Proceedings of the 26th SAS Users Group International Conference, P247-26, 1-6. Disponível em www2.sas. com/proceedings/sugi26/p247-26.pdf.

[40] Rustagi, J.S. (1994). Optimization Techniques in Statistics. San Diego: Academic Press.

[41] Saldiva, P.H.N., Lichtenfels, A.J.F.C., Paiva, P.S.O., Barone, I. A., Martins, M.A., Massad, E., Pereira, J.C.R., Xavier, V.P., Singer, J.M. e Böhm, G.M. (1994). Association between air pollution and mortality due to respiratory diseases in children in São Paulo, Brazil: a preliminary report. Environmental Research, 65, 218-225.

[42] Saldiva, P.H.N., Pope III, C.A., Schwartz, J., Dockery, D.W., Lichtenfels, A.J.F.C., Salge, J.M., Barone, I.A. e Böhm, G.M. (1995). Air pollution and mortality in elderly people: a time-series study in São Paulo, Brazil. Archives of Environmental Health, 50, 159-163.

[43] Schwartz, J. (1994). Nonparametric smoothing in the analysis of air pollution and respiratory illness. The Canadian Journal of Statistics, 22, 471-487.

[44] Schwartz, J. (1999). Air pollution and hospital admissions for heart disease in eight U.S. countries. Epidemiology, 10, 17-22.

[45] Schwartz, J., Spix, C. Touloumi, G., Bachárová, L., Barumamdzadeh, T., le Tertre, A., Piekarksi, T., Ponce de Leon, A., Pönkä, A. Rossi, G., Saez, M. e Schouten, J.P. (1996). Methodological issues in studies of air pollution and daily counts of deaths or hospital admissions. Journal of Epidemiology and Community Health, 50 (Suppl 1), S3-S11.

[46] Singer, J.M., André, C.D.S., Lima, L.P. e Conceição, G.M.S. (2002). Atmospheric Pollution and Mortality in São Paulo. Em: Statistical Data Analysis Based on the $L_{1}$-Norm and Related Methods (Statistics for Industry and Technology, Y. Dodge (Editor), 439-450. Basel: Birkhäuser. 
[47] Stone, C.J.(1977). Linear smoothers and additive models. The Annals of Statististics, 5, 595-620.

[48] Svetliza, C.F. (2002). Modelos Não-Lineares com Resposta Binomial Negativa. Tese de Doutorado: Instituto de Matemática e Estatística da Universidade de São Paulo.

[49] Svetliza, C.F. e Paula, G.A. (2001). On diagnostics in log-linear negative binomial models. Journal of Statistical Computation and Simulation, 71, 231-243.

[50] Thurston, S. W., Wand, M. P. e Wiencke, J. K. (2000). Negative Binomial Additive Models. Biometrics, 56, 139-144.

[51] Venables, W. N. e Ripley, B. D. (1997). Modern Applied Statistics with SPlus. 2a edição. New York: Springer.

[52] Wedderburn, R. W. M. (1974). Quasi-likelihood functions, generalized linear models and the Gauss-Newton method. Biometrika, 61, 439-447.

[53] Wood, S. e Lonergan, M. (2002). Generalized Additive Models using Negative Binomial errors with unknown theta. Disponivel em http://stat.ethz.ch/R-alpha/R-patched/library/mgcv/html/ gam.nbut.html

[54] Zeger, S.L. e Liang, K.Y. (1986). Longitudinal data analysis for discrete and continuous outcomes. Biometrics, 42, 121-130. 